\title{
The Infrared Telescope Facility (IRTF) spectral library
}

\section{New indices in $\mathrm{Y}, \mathrm{J}, \mathrm{H}$, and $\mathrm{L}$ atmospheric windows ${ }^{\star}$}

\author{
L. Morelli ${ }^{1}$, V. D. Ivanov ${ }^{2}$, A. Pizzella ${ }^{3,4}$, D. Gasparri ${ }^{1}$, L. Coccato ${ }^{2}$, E. M. Corsini ${ }^{3,4}$, E. Dalla Bontà ${ }^{3,4}$, \\ P. François ${ }^{5}$, and M. Cesetti ${ }^{6}$
}

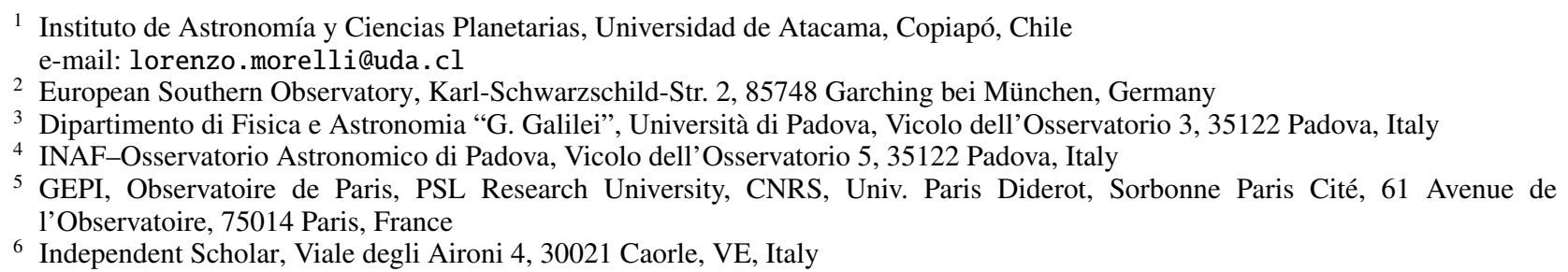

Received 14 January 2020 / Accepted 17 June 2020

\begin{abstract}
Context. Stellar population studies in the infrared (IR) wavelength range have two main advantages with respect to the optical regime: they probe different populations, because most of the light in the IR comes from redder and generally older stars, and they allow us to see through dust because IR light is less affected by extinction. Unfortunately, IR modeling work was halted by the lack of adequate stellar libraries, but this has changed in the recent years.

Aims. Our project investigates the sensitivity of various spectral features in the $1-5 \mu \mathrm{m}$ wavelength range to the physical properties of stars $\left(T_{\mathrm{eff}},[\mathrm{Fe} / \mathrm{H}], \log g\right)$ and aims to objectively define spectral indices that can characterize the age and metallicity of unresolved stellar populations.

Methods. We implemented a method that uses derivatives of the indices as functions of $T_{\mathrm{eff}},[\mathrm{Fe} / \mathrm{H}]$ or $\log g$ across the entire available wavelength range to reveal the most sensitive indices to these parameters and the ranges in which these indices work.

Results. Here, we complement the previous work in the $I$ and $K$ bands, reporting a new system of $14,12,22$, and 12 indices for $\mathrm{Y}, \mathrm{J}, \mathrm{H}$, and L atmospheric windows, respectively, and describe their behavior. We list the equivalent widths of these indices for the Infrared Telescope Facility (IRTF) spectral library stars.

Conclusions. Our analysis indicates that features sensitive to the effective temperature are present and measurable in all the investigated atmospheric windows at the spectral resolution and in the metallicity range of the IRTF library for a signal-to-noise ratio greater than 20-30. The surface gravity is more challenging and only indices in the $\mathrm{H}$ and $\mathbf{J}$ windows are best suited for this. The metallicity range of the stars with available spectra is too narrow to search for suitable diagnostics. For the spectra of unresolved galaxies, the defined indices are valuable tools in tracing the properties of the stars in the IR-dominant stellar populations.
\end{abstract}

Key words. infrared: stars - line: identification - stars: abundances - supergiants - stars: late-type - stars: fundamental parameters

\section{Introduction}

The extension of stellar evolutionary models towards the observationally challenging infrared (IR) regime is driven by the fact that optical and IR light originate from different types of stars, allowing us to obtain independent constraints on young and old stellar populations. Another reason to venture into the IR region is to penetrate the dust in galaxies with heavy extinction (e.g., Engelbracht et al. 1998; Ivanov et al. 2000). Furthermore, new sets of galaxy spectra with better quality than before become available in the IR, posing further challenges to modeling (e.g., François et al. 2019).

The interpretation of these data requires both understanding of the complex physical processes in galaxies and a significant observational effort to build libraries of IR stellar spectra. In recent times, technological improvements have allowed

* Full Tables E.1-E.6 are only available at the CDS via anonymous ftp to cdsarc.u-strasbg.fr $(130.79 .128 .5)$ or via http: //cdsarc.u-strasbg.fr/viz-bin/cat/J/A+A/641/A44 significant advances in both these areas. The Infrared Telescope Facility (IRTF) stellar library (Cushing et al. 2005; Rayner et al. 2009) offered 226 stellar and brown dwarf spectra with a resolving power of $R \sim 2000$ over $\lambda=1-5 \mu \mathrm{m}$. Conroy \& van Dokkum (2012), Röck et al. (2015, 2016), and Vazdekis et al. (2016) incorporated the IRTF library into their models. To improve the short metallicity range of the IRTF library, Villaume et al. (2017) presented a new IRTF library with an extended metallicity range $(-1.7 \leq[\mathrm{Fe} / \mathrm{H}] \leq 0.6 \mathrm{dex})$. This library allows us to expand the stellar population models to wide metallicity and wavelength ranges (Conroy et al. 2018).

The X-shooter Spectral Library (XSL, Chen et al. 2011; Arentsen et al. 2019) and its later releases (Chen et al. 2014; Gonneau et al. 2020) also extends into the IR $(\lambda=0.3-2.5 \mu \mathrm{m})$, although with a somewhat higher resolving power of $R \sim 10000$. In Cesetti et al. (2013, hereafter Paper I), we investigated the sensitivity of the features in the I and $\mathrm{K}$ atmospheric windows to the stellar physical parameters using the method of "sensitivity functions" - derivatives of the changes in the spectra with 
respect to the effective temperature $T_{\text {eff }}$, surface gravity $\log g$, and to some extent (because of the limited metallicity spread in the IRTF library) to the metallicity $[\mathrm{Fe} / \mathrm{H}]$. Indeed, the hydrogen, $\mathrm{Na}, \mathrm{Ca}$, and $\mathrm{CO}$ features turned out to be the best $T_{\text {eff }}$ indicators, and we highlighted that some $\mathrm{Mg}$ and Fe features can serve the same purpose. We confirmed that the well-known $\mathrm{Ca}$ lines and $\mathrm{CO}$ bands were the best $\log g$ indicators and we demonstrated the surface gravity sensitivity of the FeClTi feature at $0.908-0.910 \mu \mathrm{m}$. The most promising abundance indicators were of course the $\mathrm{Ca}, \mathrm{Fe}$, and $\mathrm{Mg}$ lines, but the limited $[\mathrm{Fe} / \mathrm{H}]$ range (from -0.6 to $0.4 \mathrm{dex}$ ) prevented us from deriving firm conclusions.

In this paper, we complete the exploration of the IRTF libraries, defining indices optimized for stellar population analysis in the $\mathrm{Y}, \mathrm{J}, \mathrm{H}$, and $\mathrm{L}$ atmospheric windows. Our work constitutes a preparation for the wealth of data expected from the new generation of instruments like the Multi-Object Optical and Near-IR Spectrograph (MOONS; Cirasuolo et al. 2011) at the Very Large Telescope, the Infrared Multi-Object Spectrograph (IRMS; Mobasher et al. 2010) at the Thirty Meter Telescope, and the ELT Adaptive optics for GaLaxy Evolution (EAGLE; Cuby et al. 2010) at the Extreme Large Telescope (ELT) that will make it possible to measure spectral features of $\alpha$ - and iron-group elements of a large number of galaxies, and even of individual red super giants to tens of megaparsecs (Evans et al. 2011). Here, we provide the basis for these near-future facilities. Furthermore, we also investigate the mid-IR L window features that will be accessible with the James Webb Space Telescope.

Section 2 briefly describes the properties of the IRTF spectral library. The main spectral features of the Y, J, K, and L atmospheric windows, sensitivity map analysis, and their use as spectral diagnostics are discussed in Sects. 3, 4, and 7, respectively. Index definition and measurements and their sensitivity to the galaxy broadening velocity are described in Sects. 5 and 6 respectively. Section 8 describes and discusses our results. Due to the large number of results we included in the text only those for the $Y$ band, and we moved all the figures for the $J, H$, and $L$ bands to Sect. 8. The plots of the model spectra and of the sensitivity maps are shown in Appendices A and B respectively. The plots of the broadening effects are shown in Appendix $\mathrm{C}$ and the behavior of the spectral indices in Appendix D. Finally the values of the measured indices in the different bands are reported in Appendix E.

\section{IRTF spectral library and analysis method}

The IRTF spectral library (Cushing et al. 2005; Rayner et al. 2009) contains flux calibrated spectra of about 200 objects, with a typical uncertainty of about $5 \%$. The analysis of the spectra and methodology adopted in this work are the same as in Paper I. In this section, we give a short summary, while we refer to Paper I for a detailed description of the performed steps.

The spectra were re-binned and normalized to unity at regions free of absorption features, in order to reduces the scatter in our final analysis. We then assembled the available spectral types (Spt), $T_{\text {eff }}, \log g$, [Fe/H], $M_{V}$, and parallaxes for the sample stars from the literature (Paper I, Table 3), typically derived from high-resolution optical spectroscopy.

Following the convention adopted in Paper I, we quantified the spectral types with two parametrizations. One is based on the corresponding effective temperature and the other on a quantitative analog SpT of the spectral classes, as follows: $\mathrm{SpT}=0$ for F0 stars, 1 for G0, 2 for K0, and so on, with decimals for the sub-types (e.g., SpT = 0.5 for F5; 1.8 for G8; 3.05 for M0.5).
Our method is a generalization of the sensitivity indices applied first by Worthey et al. (1994, see their Tables 2 and 3), which are simple derivatives of the index strength with respect to the physical parameter of interest (e.g., $T_{\text {eff }},[\mathrm{Fe} / \mathrm{H}], \log g$ ): a positive/negative sensitivity index implies that the feature becomes stronger/weaker with an increasing parameter value, while zero means no dependence.

We improved this method, applying it directly to the spectra in the wavelength space. To provide continuity across the entire parametric space we first generated semi-continuous sets of interpolated spectra (called model spectra in Paper I), which were constructed by least-square fitting of the normalized intensity with a second-order polynomial as a function of spectral type (SpT), surface gravity, or temperature for each wavelength bin independently, and then combining in the wavelength space the polynomial fits for all wavelength bins. Finally, the sensitivity map was obtained by calculating the derivatives of the interpolated spectra with respect to spectral type, surface gravity, or temperature.

Following the sensitivity map, we identify spectral regions sensitive to a given physical parameter (they appear as sharp features on the respective sensitivity map) and we defined the indices accordingly to encompass these regions. Adopting the same approach as Paper I, we decided to define narrow width indices instead of intermediate width indices. Broader indices do boost the signal-to-noise ratio $(\mathrm{S} / \mathrm{N})$, but they were mostly investigated with lower resolution spectrographs (e.g., Wing \& Spinrad 1970; Lançon et al. 2007) of the past generation, so we decided to concentrate on the narrow width indices taking advantage of the higher resolution of our spectra.

It should be noticed that in Paper I we were forced to limit the analysis of the metallicity effects, because of the narrow $[\mathrm{Fe} / \mathrm{H}]$ range spanned by the IRTF stars. We decided to adopt the same library in this second paper to perform an homogeneous analysis in all the atmospheric windows. In a forthcoming paper, we will extend the investigation of the metallicity effects to the wider $[\mathrm{Fe} / \mathrm{H}]$ range spanned by the new extended IRTF stellar library (Villaume et al. 2017).

\section{Main spectral features in $\mathrm{Y}, \mathrm{J}, \mathrm{H}$, and $\mathrm{L}$ atmospheric windows}

In this section we give a brief review of the charachteristics of the atmospheric windows considered in the paper and we describe their main spectral features.

\section{1. $Y$ atmospheric window}

The spectral region around $1 \mu \mathrm{m}$ has long been neglected, because it falls in between the optical and IR regimens where for a long time detector technology did not work well. The photometric $Y$ filter centered at $1.035 \mu \mathrm{m}$ (with a full whidth half maximum (FWHM) of $\sim 0.13 \mu \mathrm{m}$ ) was introduced by Hillenbrand et al. (2002) to take advantage of the better atmospheric transmission in that region. They also investigated which features dominate the spectra of cool stars and brown dwarfs at $\lambda \sim 0.97-1.07 \mu \mathrm{m}$. A few low- and medium-resolution spectral libraries of late-type stars covered this spectral region (Leggett et al. 1996; Joyce et al. 1998; Lançon \& Wood 2000; McLean et al. 2003; Cushing et al. 2005; Lodieu et al. 2005). More recently, Sharon et al. (2010) published a high-resolution $(R \sim 25000)$ atlas for the full range of luminosity classes of $\mathrm{F}, \mathrm{G}, \mathrm{K}$, and $\mathrm{M}$ stars. The XSL also covers the Y window (Chen et al. 2014; Arentsen et al. 2019). 
We note that here and in the following we only mention works that include a few tens or more stars and that span many SpT and/or luminosity classes, because only these libraries are actually of interest for stellar population studies.

The strongest absorption features in the $\mathrm{Y}$ atmospheric window $(\sim 0.94-1.10 \mu \mathrm{m})$ are the $\mathrm{H}$ lines $\mathrm{Pa} \gamma, \mathrm{Pa} \delta$, and $\mathrm{Pa} \epsilon$. There are also many low and moderate excitation lines of neutral metals, such as CaI, CrI, FeI, KI, MgI, NaI, NiI, SiI, and TiI along with singly ionized SrII (Leggett et al. 1996; Lyubchik et al. 2004). The spectra show molecular bands, including the firstovertone band heads of $\mathrm{CN}$ extending redward at $1.097 \mu \mathrm{m}$ (McKellar 1988). For the later SpTs, the Y window contains TiO bands that appear at $0.982 \mu \mathrm{m}$ and $1.014 \mu \mathrm{m}, \mathrm{FeH}$ band-head appearing at $0.989 \mu \mathrm{m}$ (Leggett et al. 1996), and VO bands at $1.06 \mu \mathrm{m}$ (Leggett et al. 1996; Cushing et al. 2005; Rayner et al. 2009). Finally, broad $\mathrm{H}_{2} \mathrm{O}$ absorption bands appear on both sides of the $\mathrm{Y}$ window. Generally, the $\mathrm{H}$ lines are stronger in $\mathrm{F}$ - and G-type stars, while the metal and molecular features are more relevant in K- and M-type stars.

The Y atmospheric window interpolated spectra for the different SpTs (i.e., for different $T_{\text {eff }}$ ) for supergiant, giant, and dwarf stars are shown in Figs. 1, A.1, and A.2, respectively.

\subsection{J atmospheric window}

Photometrically, the first practical exploration of the $\mathrm{J}$ window $(\lambda \sim 1.11-1.33 \mu \mathrm{m})$ dates back to Fellgett (1951). Johnson (1966) defined the precursors of the JHK filters used today. The $\mathrm{J}$ window is relatively free of telluric features compared with the other parts of the IR, and this makes it more valuable for quantitative spectroscopy. The first spectroscopic atlas of stars observed in the IR was published by Johnson \& Méndez (1970). A number of other spectral libraries covered it before the IRTF library (Leggett et al. 1996; Joyce et al. 1998; Lançon \& Wood 2000; McLean et al. 2003; Lodieu et al. 2005; Lançon et al. 2007; Ranade et al. 2007; Lebzelter et al. 2012). The work of Wallace et al. (2000) stands out because their spectra have higher resolving power $(R \sim 3000)$ and better $\mathrm{S} / \mathrm{N}$. The XSL also covers the J window (Chen et al. 2014).

A prominent feature of $\mathrm{F}$ and $\mathrm{G}$ stars in the $\mathrm{J}$ window is the $\mathrm{Pa} \beta$ at $1.282 \mu \mathrm{m}$, whose strength decrease toward late sub-types. It becomes very weak in $\mathrm{K}$ stars and is absent for $\mathrm{M}$ stars whose spectra are dominated by lines of neutral metal species of lower ionization potential. Notable atomic features in the spectra of $\mathrm{K}$ and $\mathrm{M}$ stars are the NaI doublet at $1.14 \mu \mathrm{m}$, the KI doublet at $1.18 \mu \mathrm{m}$ and $1.25 \mu \mathrm{m}$, and the AlI doublet at $1.313 \mu \mathrm{m}$ and $1.318 \mu \mathrm{m}$. They are strongest in late-M dwarfs and weaker in corresponding giants and supergiants. Therefore, they are excellent luminosity class (or surface gravity) indicators. The SiI features appear in early $\mathrm{F}$ stars and fade in early $\mathrm{M}$ stars. We also see some FeI, MgI, TiI, and MnI lines, but they are too weak to be suitable for extragalactic work.

At the resolution of our data, a number of spectral features are blends of absorption lines from different chemical elements. For example, the feature at $\sim 1.169 \mu \mathrm{m}$ is due primarily to $\mathrm{CI}$ in $\mathrm{F}$ and $\mathrm{G}$ stars. However, for later spectral types, KI absorption plays the dominant role in determining the feature strength. The $\mathrm{Al}, \mathrm{Mn}, \mathrm{Fe}$, and $\mathrm{Mg}$ lines behave similarly to $\mathrm{Si}$, but persist through the latest observed types. The continuum shape in the $\mathbf{J}$ window is redder toward later spectral type. The $\mathrm{J}$ atmospheric window interpolated spectra for the different SpTs (i.e., for different $\left.T_{\text {eff }}\right)$ for supergiant, giant, and dwarf stars are shown in Figs. A.3-A.5, respectively.

\subsection{H atmospheric window}

The photometric use of the $\mathrm{H}$ window $(\lambda \sim 1.48-1.78 \mu \mathrm{m})$ also goes back to Fellgett (1951) and Johnson (1966), but the spectroscopic treatment is more difficult than the $\mathrm{J}$ window because it contains more telluric absorptions. Furthermore, the background emission is due to numerous $\mathrm{O}_{2} \mathrm{H}$ and $\mathrm{OH}$ lines that vary on a timescale of a few minutes, making the data processing more challenging ${ }^{1}$.

Although the $\mathrm{K}$ window $(\lambda \sim 1.92-2.40 \mu \mathrm{m})$ is the most studied, the presence of circumstellar dust or active galactic nuclei (AGN) often results in a significant excess of continuum emission at $\lambda \geq 2 \mu \mathrm{m}$ (Schinnerer et al. 1997; Ivanov et al. 2000). This excess weakens or even renders invisible the photospheric absorption features, making the $\mathrm{K}$ window unsuitable for stellar population studies. Yet, the radiation from the stellar photospheres still dominates shorter wavelengths in the $\mathrm{H}$ window. This motivated the first studies of star spectra in the $\mathrm{H}$ window (e.g., Johnson \& Méndez 1970; Lancon \& Rocca-Volmerange 1992; Origlia et al. 1993; Dallier et al. 1996; Morris et al. 1996; Blum et al. 1997; Meyer et al. 1998). More recently, a number of spectral libraries have been reported for wide varieties of stars (e.g., Ivanov et al. 2004; Ranade et al. 2004; Lodieu et al. 2005; Hanson et al. 2005; Venkata Raman \& Anandarao 2008; Huynh et al. 2011; Cooper et al. 2013; Ghosh et al. 2019). The spectral libraries of Lebzelter et al. (2012) and Park et al. (2018) stand out because of their significantly higher resolving power of $R \sim 100000$ and 45000 , respectively. However, they contain less stars and are not really suitable for galaxy population modeling.

A large effort was devoted to abundance studies of red giants and supergiants because of the rich concentration of both molecular and metal lines in the H window (Origlia et al. 1993, 2002, 2003; Origlia \& Rich 2004; Cunha et al. 2007; Davies et al. 2009a,b). High-resolution spectra in the $H$-band domain enabled the Apache Point Observatory Galactic Evolution Experiment survey to determine the chemical abundances of 16 elements for all their stars (Smith et al. 2013; García Pérez et al. 2016; Fernández-Trincado et al. 2019).

The identification of features in the $\mathrm{H}$ window is challenging because there are many relatively weak metal absorption features and high-order $\mathrm{H}$ lines from the $\mathrm{Br}$ series and the second $\mathrm{CO}$ overtone vibrational-rotational band is present in cooler objects. Fortuitously, a few metal absorptions of MgI (at 1.49$1.51 \mu \mathrm{m}$ and $1.71 \mu \mathrm{m}), \mathrm{Si}(\sim 1.59 \mu \mathrm{m})$, and $\mathrm{Al}(\sim 1.67 \mu \mathrm{m})$ are clearly separated especially for hot stars. The $\mathrm{Mg}$ and $\mathrm{Al}$ features are the deepest in $\mathrm{K}$ and early $\mathrm{M}$ dwarfs and they are significantly weaker in giants and supergiants for a given SpT. For this reason, these lines appear to be luminosity class indicators.

$\mathrm{H}_{2} \mathrm{O}$ bands are present on both sides of the $\mathrm{H}$ atmospheric window for spectral types later than M4 (see also Rayner et al. 2009). Some $\mathrm{FeH}$ absorption features are also evident, as pointed out by Cushing et al. (2003). The second overtone vibrationalrotational band of $\mathrm{CO}$ is another good surface gravity indicator in $\mathrm{K}$ and $\mathrm{M}$ stars. Many $\mathrm{OH}$ band features are present in this spectral range. They are often in a region crowded with spectral features as shown by Lebzelter et al. (2012) and Smith et al. (2013), and could contaminate close spectral features. Therefore, we consider here only the most isolated ones to try to avoid confusion as much as possible. The $\mathrm{H}$ atmospheric window

\footnotetext{
1 For further information see the ISAAC user manual: http:// www.eso.org/sci/facilities/paranal/decommissioned/ isaac/doc/VLT-MAN-ESO-14100-0841_v92.pdf
} 


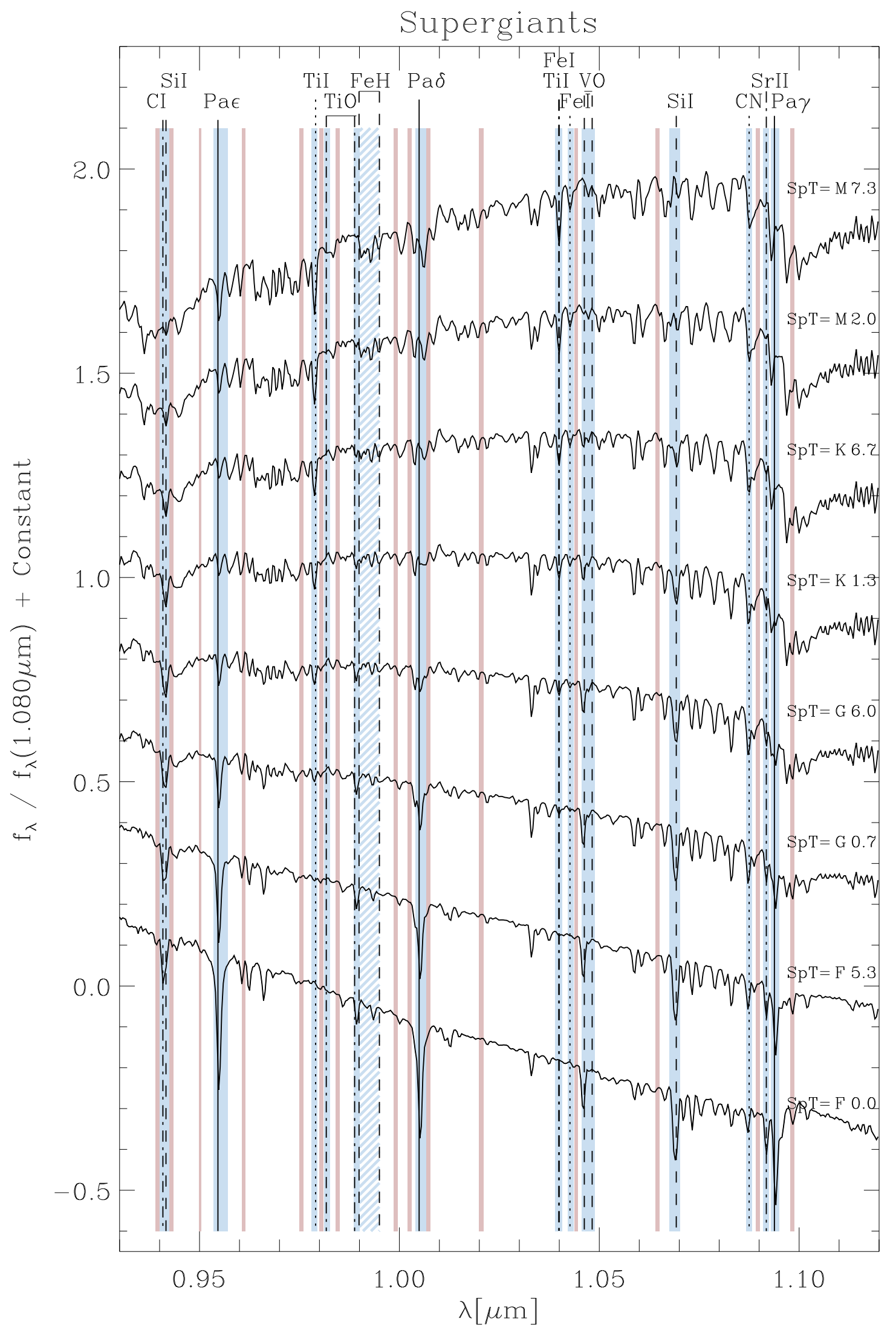

Fig. 1. Y atmospheric window model spectra of supergiants obtained by fitting at each wavelength the flux-normalized (at $1.08 \mu \mathrm{m})$ sample spectra along SpTs. The model spectra for different SpTs are offset for displaying purposes and the SpTs are given on the right. The green regions mark the bandpasses of the newly defined indices, the gray regions mark their adjacent continuum as defined in this paper (see Table 1). Some relevant absorption features are marked. Here and for the other bands we did not consider features at the edges of the windows, because they can be affected by variable atmospheric transmission. The plots for stars of other luminosity classes and atmospheric windows are shown in Appendix A. 
Table 1. Definition of the spectral indices.

\begin{tabular}{|c|c|c|c|c|c|c|c|}
\hline \multirow{2}{*}{$\begin{array}{l}\text { Index } \\
\text { name }\end{array}$} & \multirow[t]{2}{*}{ Species } & \multicolumn{2}{|c|}{ Bandpass definitions $(\mu \mathrm{m})$} & \multirow{2}{*}{$\begin{array}{l}\text { Index } \\
\text { name }\end{array}$} & \multirow[t]{2}{*}{ Species } & \multicolumn{2}{|c|}{ Bandpass definitions $(\mu \mathrm{m})$} \\
\hline & & Central & Blue and red continua & & & Central & Blue and red continua \\
\hline \multicolumn{4}{|c|}{$\mathrm{Y}$ atmospheric window } & \multicolumn{4}{|c|}{$\mathrm{H}$ atmospheric window (cont): } \\
\hline $\mathrm{CSi}$ & $\mathrm{CI}+\mathrm{SiI}$ & $0.9400-0.9424$ & $0.9390-0.93990 .9425-0.9434$ & $\mathrm{Br}_{16}$ & $\mathrm{HI}(n=4)$ & $1.552-1.557$ & $1.548-1.550 \quad 1.593-1.594$ \\
\hline $\mathrm{Pa} \epsilon$ & $\mathrm{HI}(n=3)$ & $0.9535-0.9570$ & $0.9498-0.95040 .9606-0.9614$ & $\mathrm{CO}^{*}$ & ${ }^{12} \mathrm{CO}(2,0)$ & $1.557-1.5635$ & $1.548-1.550 \quad 1.593-1.594$ \\
\hline $\mathrm{Ti}$ & TiI & $0.9780-0.9795$ & $0.9750-0.97600 .9800-0.9810$ & $\mathrm{Br}_{15}$ & HI & $1.567-1.572$ & $1.548-1.550 \quad 1.593-1.594$ \\
\hline $\mathrm{TiO} \mathrm{A}$ & $\mathrm{TiO}$ & $0.9812-0.9826$ & $0.9800-0.98100 .9840-0.9850$ & $\mathrm{Mg}^{\ddagger}$ & MgI & $1.573-1.580$ & $1.548-1.5501 .593-1.594$ \\
\hline $\mathrm{TiO} \mathrm{B}$ & $\mathrm{TiO}$ & $0.9885-0.9900$ & $0.9840-0.98500 .9985-0.9995$ & $\mathrm{FeH} 1^{\ddagger, \Delta, \square}$ & $\mathrm{FeH}$ & $1.582-1.586$ & $1.548-1.5501 .593-1.594$ \\
\hline $\mathrm{FeH}^{\dagger, \diamond}$ & $\mathrm{FeH}$ & $0.9900-0.9950$ & $0.9840-0.98500 .9985-0.9995$ & $\mathrm{Si}^{\ddagger, \Delta, \square}$ & SiI & $1.587-1.591$ & $1.548-1.5501 .593-1.594$ \\
\hline $\operatorname{Pa} \delta$ & $\mathrm{HI}(n=3)$ & $1.0040-1.0067$ & $1.0020-1.00301 .0067-1.0077$ & $\mathrm{CO} 2^{\ddagger}$ & ${ }^{12} \mathrm{CO}(2,0)$ & $1.595-1.600$ & $1.593-1.5941 .616-1.618$ \\
\hline FeTi & FeI+TiI & $1.0390-1.0408$ & $1.0198-1.02101 .0438-1.0446$ & $\mathrm{Fe} 2$ & FeI & $1.605-1.609$ & $1.593-1.5941 .616-1.618$ \\
\hline $\mathrm{Fe}$ & FeI & $1.0422-1.0436$ & $1.0198-1.02101 .0438-1.0446$ & $\mathrm{Br}_{13}$ & $\mathrm{HI}(n=4)$ & $1.610-1.614$ & $1.593-1.5941 .616-1.618$ \\
\hline VO & VO & $1.0456-1.0488$ & $1.0438-1.04461 .0640-1.0650$ & $\mathrm{CO}^{\triangle}$ & ${ }^{12} \mathrm{CO}(2,0)$ & $1.618-1.622$ & $1.616-1.6181 .634-1.637$ \\
\hline $\mathrm{Si}^{\ddagger}$ & SiI & $1.0676-1.0702$ & $1.0640-1.06501 .0892-1.0902$ & $\mathrm{FeH} 2$ & $\mathrm{FeH}$ & $1.624-1.628$ & $1.616-1.618 \quad 1.634-1.637$ \\
\hline $\mathrm{CN}$ & $\mathrm{CN}$ & $1.0868-1.0882$ & $1.0640-1.06501 .0892-1.0902$ & $\mathrm{CO} 4$ & ${ }^{12} \mathrm{CO}(2,0)$ & $1.639-1.647$ & $1.634-1.6371 .6585-1.6605$ \\
\hline $\mathrm{Sr}$ & SrII & $1.0913-1.0923$ & $1.0892-1.0902 \quad 1.0978-1.0988$ & $\mathrm{Fe} 3$ & FeI & $1.651-1.658$ & $1.634-1.6371 .6585-1.6605$ \\
\hline \multirow[t]{3}{*}{$\mathrm{Pa} \gamma$} & $\mathrm{HI}(n=3)$ & $1.0930-1.0950$ & $1.0892-1.0902 \quad 1.0978-1.0988$ & $\mathrm{CO} 5$ & ${ }^{12} \mathrm{CO}(2,0)$ & $1.6605-1.6640$ & $1.6585-1.66051 .6775-1.679$ \\
\hline & & & & Al1 & AlI & $1.6705-1.6775$ & $1.6585-1.66051 .6775-1.679$ \\
\hline & \multicolumn{3}{|c|}{$\mathrm{J}$ atmospheric window } & $\mathrm{Br}_{11}$ & $\mathrm{HI}(n=4)$ & $1.6790-1.6825$ & $1.6775-1.6791 .6825-1.6835$ \\
\hline $\mathrm{Na}^{\ddagger}, \dagger$ & NaI & $1.1358-1.1428$ & $1.1320-1.13401 .1430-1.1460$ & $\mathrm{COMg}^{\square}$ & ${ }^{12} \mathrm{CO}(2,0)$ & $1.705-1.713$ & $1.692-1.6961 .714-1.716$ \\
\hline $\mathrm{FeCr}^{\ddagger}$ & $\mathrm{FeI}+\mathrm{CrI}$ & $1.1600-1.1624$ & $1.1560-1.15851 .1716-1.1746$ & & $+\mathrm{MgI}$ & & \\
\hline $\mathrm{K} 1 \mathrm{~A}^{\dagger, \diamond}$ & KI & $1.1670-1.1714$ & $1.1560-1.15851 .1716-1.1746$ & $\mathrm{Br}_{10}$ & $\mathrm{HI}(n=4)$ & $1.735-1.739$ & $1.725-1.728 \quad 1.744-1.748$ \\
\hline $\mathrm{C}$ & CI & $1.1746-1.1765$ & $1,1716-1.17461 .1805-1.1815$ & & & & \\
\hline $\mathrm{K} 1 \mathrm{~B}^{\dagger, \diamond}$ & KI & $1.1765-1.1800$ & $1.1716-1.17461 .1805-1.1815$ & \multicolumn{4}{|c|}{$\mathrm{L}$ atmospheric window } \\
\hline $\mathrm{Mg}^{\ddagger}$ & $\mathrm{MgI}$ & $1.1820-1.1840$ & $1.1805-1.18151 .1855-1.1875$ & $M g 1^{\ddagger}$ & & $3.388-3.406$ & $3.360-3.3763 .425-3.440$ \\
\hline $\mathrm{Si}^{\ddagger}$ & SiI & $1.1977-1.2004$ & $1.1910-1.19351 .2050-1.2070$ & $\mathrm{Mg} 2^{\ddagger}$ & MgI & $3.454-3.464$ & $3.425-3.4403 .476-3.496$ \\
\hline $\mathrm{SiMg}$ & $\mathrm{SiI}+\mathrm{MgI}$ & $1.2070-1.2095$ & $1.2050-1.20701 .2150-1.2180$ & $\mathrm{P}_{16}$ & $\mathrm{OH}(1-0) \mathrm{P}_{16}$ & $3.514-3.532$ & $3.476-3.4963 .540-3.560$ \\
\hline $\mathrm{K} 2 \mathrm{~A}^{\ddagger}, \diamond$ & $\mathrm{KI}$ & $1.2415-1.2455$ & $1.2350-1.23801 .2460-1.2490$ & $\mathrm{P}_{14}$ & $\mathrm{OH}(2-1) \mathrm{P}_{14}$ & $3.564-3.582$ & $3.540-3.5603 .604-3.612$ \\
\hline $\mathrm{K} 2 \mathrm{~B}^{\diamond}$ & KI & $1.2495-1.2540$ & $1.2460-1.24901 .2560-1.2580$ & $\mathrm{P}_{17}$ & $\mathrm{OH}(1-0) \mathrm{P}_{17}$ & $3.582-3.597$ & $3.540-3.5603 .604-3.612$ \\
\hline $\mathrm{Pa} \beta^{\ddagger}$ & $\mathrm{HI}(n=3)$ & $1.2795-1.2840$ & $1.2755-1.27801 .2855-1.2873$ & $\mathrm{P}_{15}$ & $\mathrm{OH}(2-1) \mathrm{P}_{15}$ & $3.628-3.648$ & $3.604-3.6123 .665-3.673$ \\
\hline \multirow[t]{3}{*}{$\mathrm{Al}^{+, \dagger}$} & AlI & $1.3115-1.3168$ & $1.3050-1.30751 .3230-1.3250$ & Pf $\gamma$ & $\mathrm{HI}(n=5)$ & $3.736-3.745$ & $3.718-3.7283 .793-3.803$ \\
\hline & & & & $\mathrm{Mg}^{\ddagger}$ & MgI & $3.862-3.870$ & $3.840-3.8503 .870-3.880$ \\
\hline & \multicolumn{3}{|c|}{$\mathrm{H}$ atmospheric window } & $\mathrm{Hu}_{15}$ & $\mathrm{HI}(n=6)$ & $3.900-3.914$ & $3.870-3.8803 .914-3.922$ \\
\hline$M g 1^{\ddagger}$ & MgI & $1.485-1.491$ & $1.483-1.4851 .491-1.500$ & & $\mathrm{SiO}(2,0)$ & $3.998-4.012$ & $3.990-3.9984 .025-4.035$ \\
\hline $\mathrm{Mg} 2^{\ddagger, \square}$ & $\mathrm{MgI}$ & $1.500-1.508$ & $1.491-1.508 \quad 1.510-1.512$ & $\mathrm{SiO} 2$ & $\mathrm{SiO}(3,1)$ & $4.035-4.050$ & $4.025-4.0354 .060-4.070$ \\
\hline $\mathrm{K} 1$ & $\mathrm{KI}$ & $1.515-1.520$ & $1.510-1.512 \quad 1.523-1.525$ & $\mathrm{SiO} 3$ & $\mathrm{SiO}(4,2)$ & $4.080-4.090$ & $4.060-4.0704 .095-4.105$ \\
\hline $\mathrm{Fe} 1$ & FeI & $1.528-1.533$ & $1.523-1.5251 .548-1.550$ & & & & \\
\hline
\end{tabular}

Notes. We have marked with the symbol $\ddagger$ the index definitions similar to Riffel et al. (2019), with the symbol $\Delta$ those similar to Origlia et al. (1993), with the symbol $\square$ those similar to Ivanov et al. (2004), with the symbol $\dagger$ those similar to Conroy \& van Dokkum (2012) and Villaume et al. (2017), and with the symbol $\diamond$ those similar to McLean et al. (2003) and Cushing et al. (2005).

interpolated spectra for the different $\operatorname{SpTs}$ (i.e., for different $T_{\text {eff }}$ ) for supergiant, giant, and dwarf stars are shown in Figs. A.6-A.8 respectively.

\subsection{L atmospheric window}

The $\mathrm{L}$ atmospheric window $(\lambda \sim 3.35-4.15 \mu \mathrm{m})$ was first explored by Fellgett (1951) and Johnson (1966) too and a review of other early works can be found in Merrill \& Ridgway (1979). Other spectral libraries in this range were presented by Ridgway et al. (1984), Wallace \& Hinkle (2002), Mennickent et al. (2009), and Nicholls et al. (2017). Some space-based libraries of $~ 300$ stars are also available from the Infrared Space Observatory (Vandenbussche et al. 2002; Heras et al. 2002; Sloan et al. 2003) and from the Akari mission (Shimonishi et al. 2013, albeit with resolving power of $R \sim 20$ only).

The most prominent metal features belong to $\mathrm{Mg}$ at $\lambda \sim$ $3.39 \mu \mathrm{m}$ and $\lambda \sim 3.46 \mu \mathrm{m}$, while the strongest molecular bands belong to the $\operatorname{SiO}(\lambda \sim 4.00 \mu \mathrm{m}, \lambda \sim 4.06 \mu \mathrm{m}$, and $\lambda \sim 4.08 \mu \mathrm{m})$. Some weaker $\mathrm{OH}$ bands are also present. All these features are stronger in cooler stars. The molecular lines are stronger in supergiants and giants, than in dwarf stars. The blue edge of the $\mathrm{L}$ window can be affected by broad $\mathrm{H}_{2} \mathrm{O}$ absorption for M6-M9 giant stars. We exclude from our analysis the spectral region with $\lambda \lesssim 3.4 \mu \mathrm{m}$ because it is affected by poor atmospheric transmission.

Importantly, the $\mathrm{L}$ window can be significantly affected by dust emission that is circumstellar, when individual stars are considered, or in the galactic environment when galaxies are considered. The $\mathrm{L}$ atmospheric window interpolated spectra for different SpTs (i.e., for different $T_{\text {eff }}$ ) for supergiant, giant, and dwarf stars are shown in Figs. A.9-A.11, respectively.

\section{Sensitivity maps}

The sensitivity maps for the different SpTs and surface gravity according to their class and spectral type are shown in Fig. 2 and in Appendix B. The different plots are shown with a constant vertical offset for display purposes and the position of the promisingly sensitive elements is marked. L stars were excluded 


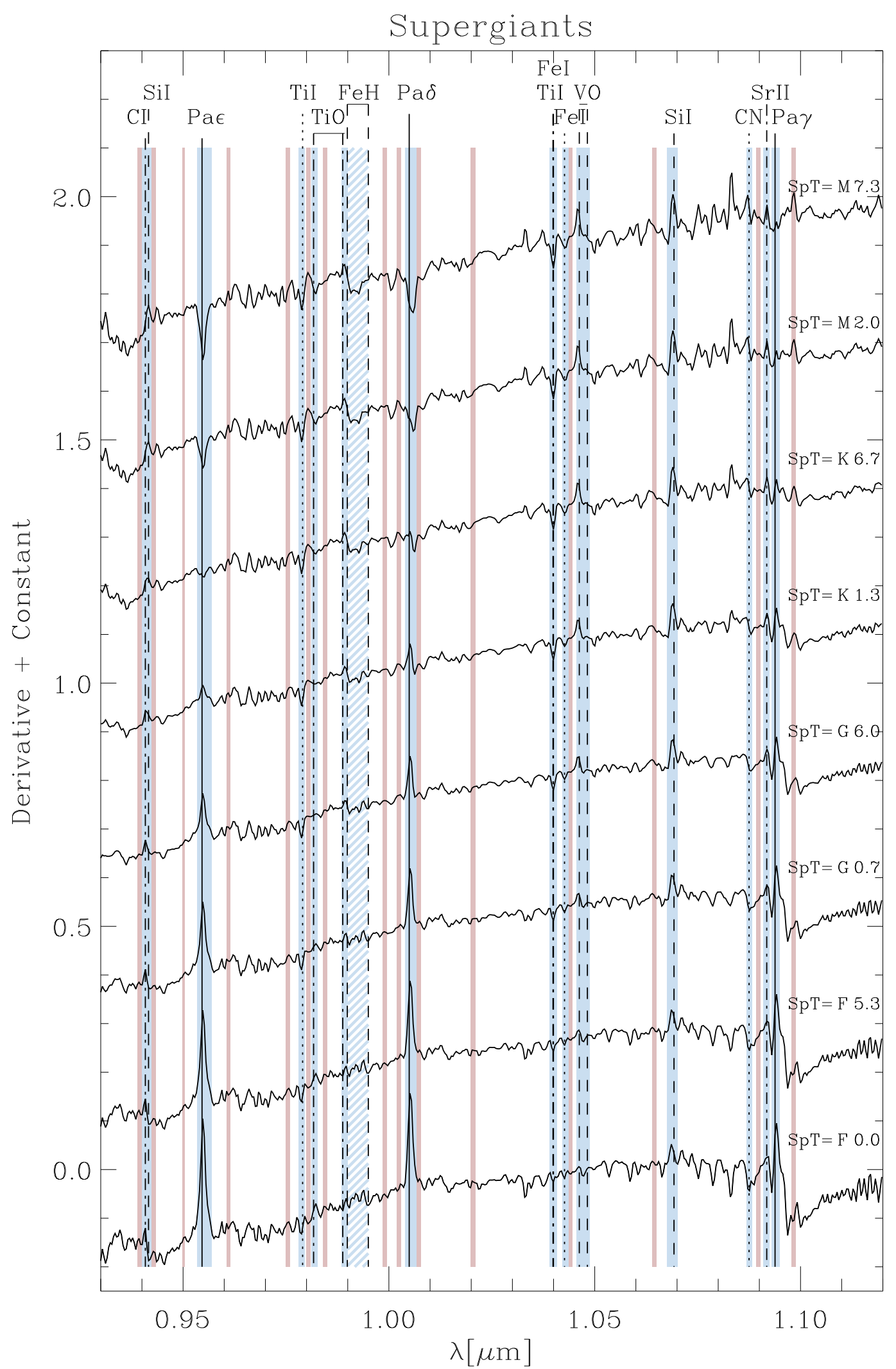

Fig. 2. Y atmospheric window sensitivity map for SpT of supergiants. Details are given in Fig. 1. The plots for other luminosity classes and atmospheric windows are shown in Appendix A.

from the surface gravity sensitivity maps because of their limited gravity coverage. At the edge of the atmospheric window, the derivative was very noisy and we exclude the lines in this range from the analysis.

\subsection{Y atmospheric window}

The $\mathrm{Y}$ atmospheric sensitivity maps with respect to the SpTs are shown in Figs. 2, B.1, and B.2 for supergiant, giant, and 
dwarf stars, respectively. The regions corresponding to the $\mathrm{Pa}$ series of the $\mathrm{H}$ are sensitive to the spectral type, showing a positive peak for the F stars that gradually transforms into a negative peak for the $\mathrm{M}$ stars. A similar trend is also shown in the $\mathrm{FeH}$ region and, even if with less intensity, in the VO band. This behavior is more evident for dwarf than for supergiant and giant stars. The other elements show much weaker features in the sensitivity map (i.e., much weaker change in the spectrum). Si and Sr seem to be sensitive only for K and M stars, while the $\mathrm{CN}$ is very close to a Fe line and they are probably contaminating each other, introducing some instability into the trend.

The Y atmospheric sensitivity maps with respect to the surface gravity are shown in Figs. 3 and 4 for the F and G stars and $\mathrm{K}$ and $\mathrm{M}$ stars, respectively.

The lines of the $\mathrm{Pa}$ series of the $\mathrm{H}$ show a positive peak increasing from the low-gravity to high-gravity stars and being evident only for the $\mathrm{G}$ and $\mathrm{F}$ type stars. Some minor but significant changes have been found in the derivative plot of the Si and the Sr lines.

\subsection{J atmospheric window}

The $\mathbf{J}$ atmospheric sensitivity maps with respect to the SpTs are shown in Figs. B.3-B.5 for supergiant, giant, and dwarf stars, respectively. In this atmospheric window the behavior of supergiant and giant stars is different from that of the dwarf stars. The $\mathrm{Na}$ doublet, $\mathrm{K}, \mathrm{Mg}, \mathrm{Si}$, and to a small extent $\mathrm{Fe}$ and $\mathrm{Al}$ show a very strong positive peak that decreases to very negative values moving from the early- to late-type dwarf stars. This trend is not visible for the supergiants and giant stars. In contrast, for supergiant and giant stars the most prominent features are $\mathrm{Pa} \beta$ and $\mathrm{C}$ with values that are positive for the F stars and decrease moving to the M stars. These changes are, instead, almost negligible for dwarf stars.

The $\mathrm{J}$ atmospheric sensitivity maps with respect to the surface gravity are shown in Figs. B.12 and B.13 for the F and G stars and $\mathrm{K}$ and $\mathrm{M}$ stars, respectively. Decreasing positive values from low-gravity to high-gravity stars for the $\mathrm{C}$ and $\mathrm{Si}$ elements were found in F and $\mathrm{G}$ stars, while $\mathrm{Na}$ and $\mathrm{K}$ elements show a dependence with gravity only in $\mathrm{K}$ and $\mathrm{M}$ stars.

\section{3. $H$ atmospheric window}

The $\mathrm{H}$ atmospheric sensitivity maps with respect to the SpTs are shown in Figs. B.6-B.8 for supergiant, giant, and dwarf stars, respectively. This is the atmospheric window with the strongest sensitivity, no matter which class of stars we consider. However, the elements showing sensitivity in giant and supergiant stars are different from the elements showing sensitivity for dwarf stars. For the latter the most evident changes are in the $\mathrm{Mg}, \mathrm{Si}, \mathrm{Fe}$, and Al lines, while the $\mathrm{Br}$ lines do not display any significant peak. On the other hand, in sensitivity maps of the giant and supergiant stars the $\mathrm{Br}$ lines have a large range of variations, going from positive values in $\mathrm{F}$ stars to negative values in $\mathrm{M}$ stars. Even if less evident than in dwarf stars, the $\mathrm{Mg}, \mathrm{Si}, \mathrm{Fe}$, and $\mathrm{Al}$ lines still maintain a weak sensitivity trend with the spectral type also in these classes.

The $\mathrm{H}$ atmospheric sensitivity maps with respect to the surface gravity are shown in Figs. B.14 and B.15 for F and G stars and $\mathrm{K}$ and $\mathrm{M}$ stars, respectively. In the $\mathrm{F}$ and $\mathrm{G}$ stars the sensitivity maps show strong variations for the $\mathrm{Br}$ lines. Their peaks are always very positive indicating strong changes in the line width. The other elements have smaller variations not comparable with those of $\mathrm{H}$ lines but still detectable. Regarding the $\mathrm{F}$ and $\mathrm{G}$ stars, the dominant variations are detected in the $\mathrm{Mg}$ and $\mathrm{CO}$ lines, while the $\mathrm{Br}$ lines show minor variations.

\subsection{L atmospheric window}

The L atmospheric sensitivity maps with respect to the SpTs are shown in Figs. B.9-B.11 for supergiant, giant, and dwarf stars, respectively. The most prominent feature is $\mathrm{Mg}$, which decreases from the $\mathrm{F}$ to the $\mathrm{M}$ stars. For the $\mathrm{Mg}$ at $\lambda \simeq 3.4 \mu \mathrm{m}$ we found a possible contamination that needs to be considered. The $\mathrm{SiO}$ also shows a clear peak in the sensitivity map decreasing from $\mathrm{F}$ stars to $\mathrm{M}$ stars. The $\mathrm{P}$ lines show medium to weak sensitivity to the changes along SpT.

The $\mathrm{L}$ atmospheric sensitivity maps with respect to the surface gravity are shown in Figs. B.16 and B.17 for F and G stars and $\mathrm{K}$ and $\mathrm{M}$ stars, respectively. $\mathrm{F}$ and $\mathrm{G}$ stars show a weak dependency of the $\mathrm{SiO}$ lines with the surface gravity and almost no sensitivity has been detected in the sensitivity map of $\mathrm{F}$ and K stars.

\section{Indices definition and equivalent width measurements}

We defined an index for all the features that display a significant variation in the sensitivity maps (at least over some range of parameters), and that are therefore promising tools to measure $T_{\text {eff }}$ (or Spt) and $\log g$. For each index, we have a central bandpass covering the feature of interest and two adjacent bandpasses tracing the local "continuum"2 at the red and blue sides of the feature, as done in Paper I. The equivalent width for each spectral feature has been derived following Eq. (1) in Paper I. The central bandpass was designed to include the peak of the particular feature at the sensitivity map and the continuum bandpasses were placed on spectral regions where the sensitivity map is, as much as possible, constant.

We defined 14 indices in Y, 12 in J, 22 in H, and 12 in the $\mathrm{L}$ atmospheric windows. The index definitions are listed in Table 1 and their bandpasses are plotted in Figs. 1, 4, and A.1-A.11. We measured the equivalent widths of all the indices. Their values are listed in Tables E. 2 and E.6 and plotted versus the star effective temperature and surface gravity in Figs. 5, 6, and D.1-D.7, respectively.

The precision of the measured indices depends on the $\mathrm{S} / \mathrm{N}$ of the spectra, which varies widely because of the sky emission and telluric absorption features. To obtain a robust evaluation of the uncertainties of the indices, we simulated some indices of the $Y$ band, choosing as example $\mathrm{CSi}, \mathrm{Pa} \epsilon$, and $\mathrm{Pa} \delta$ for a FII star, Ti, CN, and $\mathrm{Pa} \delta$ for a KIII star, and $\mathrm{FeH}, \mathrm{VO}$, and $\mathrm{Pa} \delta$ for a MI star. We generated spectra with eight different $\mathrm{S} / \mathrm{N}$ levels, ranging from 20 to 120 , by adding artificial noise to the three test stars from the IRTF library.

Figure 7 shows the effect of the $\mathrm{S} / \mathrm{N}$ on the precision of the measured indices. Generally, it is negligible except for very low $\mathrm{S} / \mathrm{N}(<10)$. For some features (e.g., $\mathrm{Pa} \epsilon$ in FII star or FeH in MI star), we can obtain a precision of $10 \%$ for $S / N \sim 10-20$, while for others (e.g., $\mathrm{Pa} \delta$ in MI star or $\mathrm{CN}$ in KIII star) we can reach a precision of $30 \%$ for $S / N \sim 20-40$. Only for a few weaker features, the precision remains at a $50 \%$ level even for higher $\mathrm{S} / \mathrm{N}$.

2 We note that at the IRTF library resolution these bands do not actually measure the stellar continuum in cool stars. 
A\&A 641, A44 (2020)

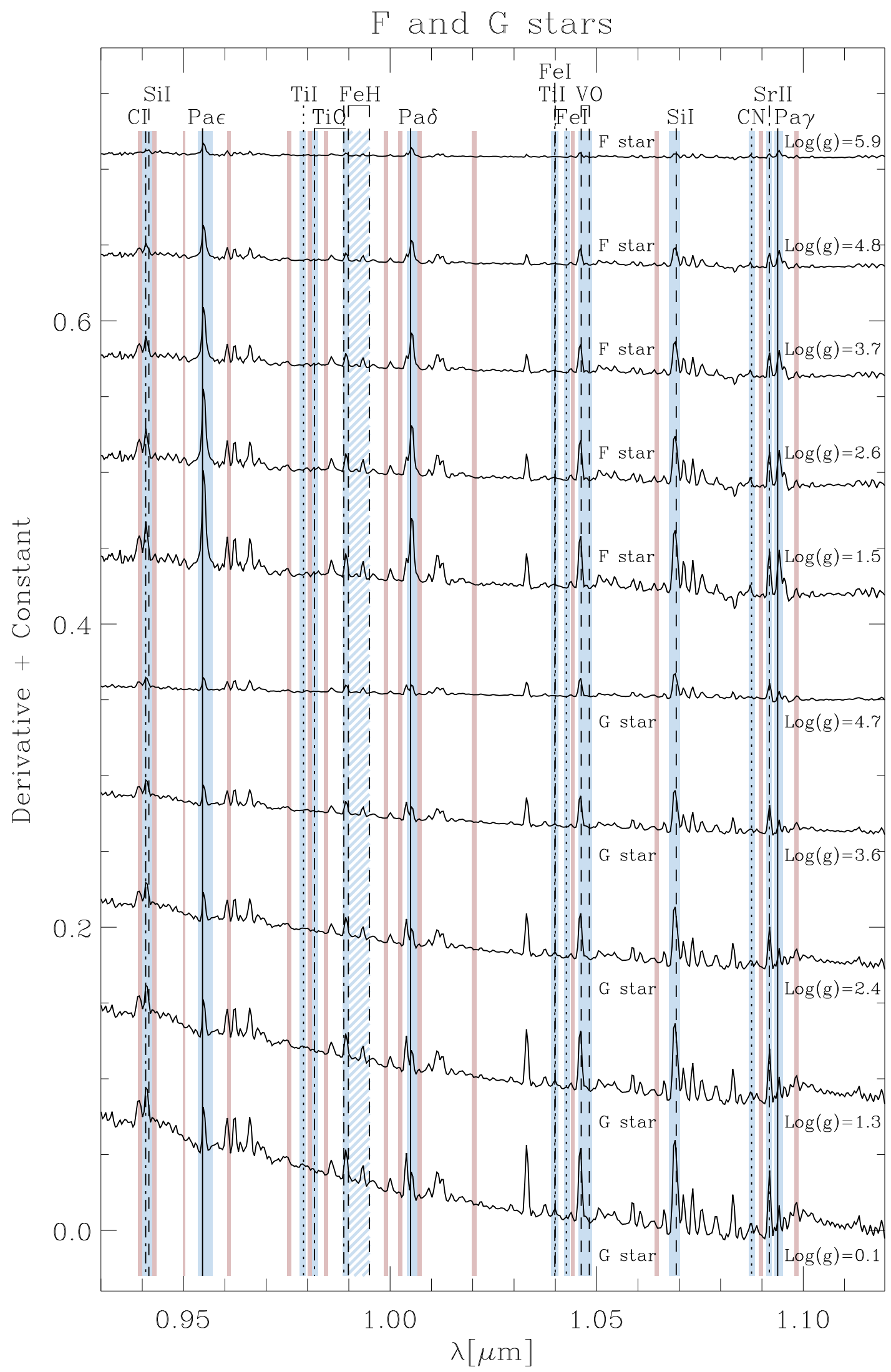

Fig. 3. Y atmospheric window sensitivity map for surface gravity of F (top) and G-type stars (bottom). The sensitivities for different gravity values are offset for display purposes and the central values of the corresponding $\log g$ bins are given. Figure 2 gives more details. 
L. Morelli et al.: The Infrared Telescope Facility (IRTF) spectral library. II.

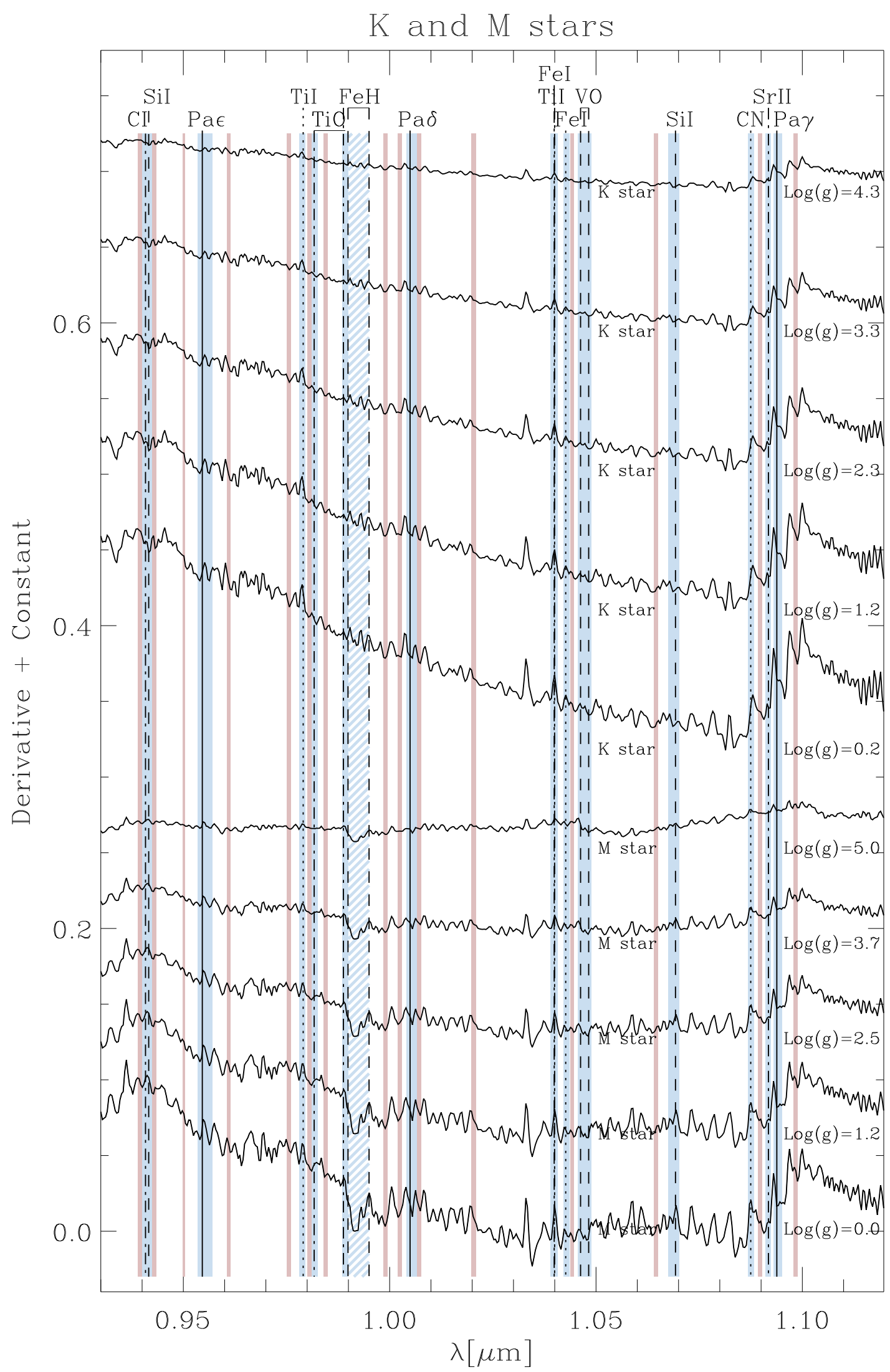

Fig. 4. Y atmospheric window sensitivity map for surface gravity of K (top) and M-type stars (bottom). Figure 3 gives more details. 

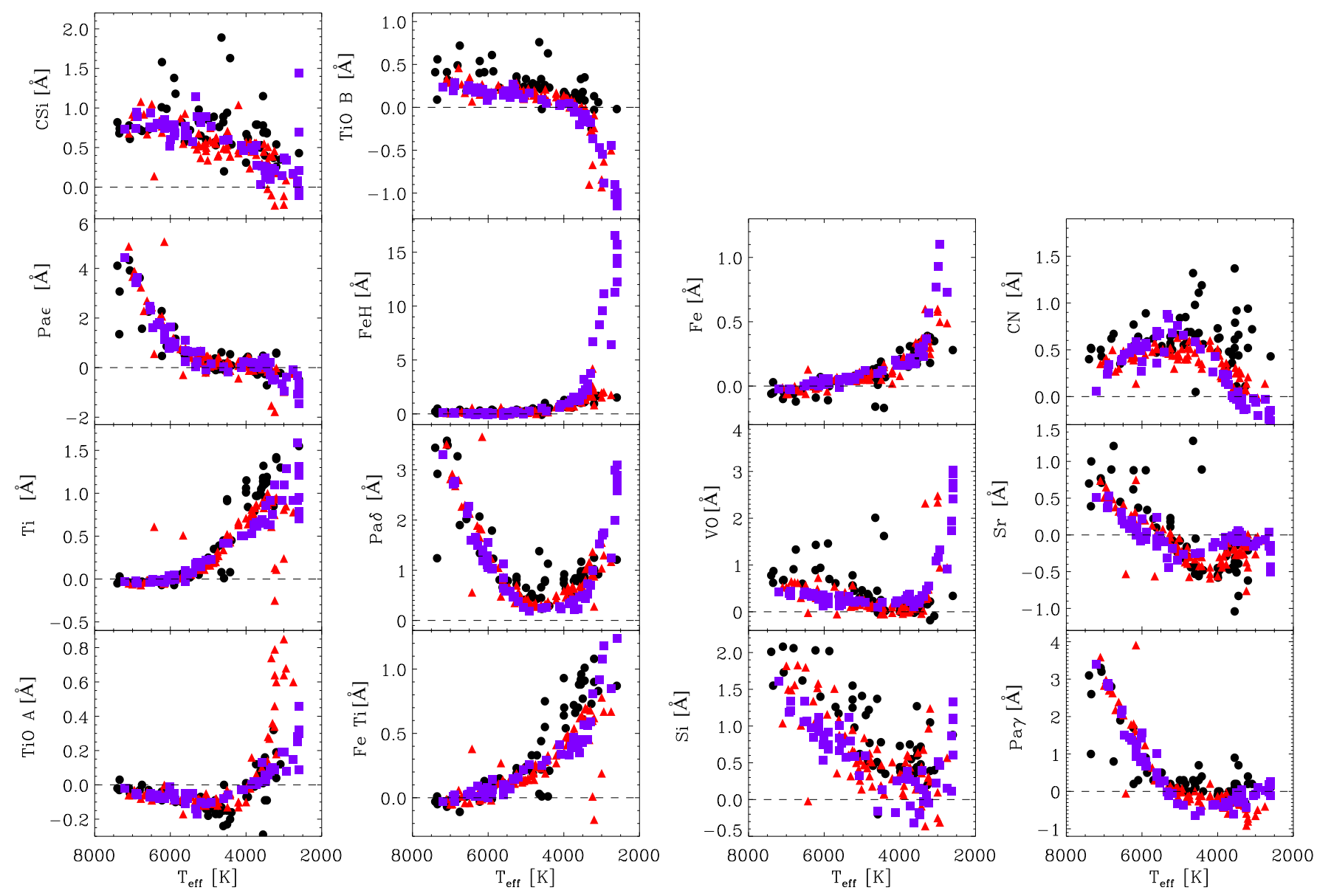

Fig. 5. Index measurements in the $\mathrm{Y}$ atmospheric window as functions of effective temperature. The different symbols correspond to the supergiants (circles), giants (triangles), and dwarf stars (squares), respectively.

\section{Sensitivity of indices to galaxy velocity dispersion broadening}

The application of spectroscopic index systems to study stellar populations of unresolved galaxies (e.g., Dressler et al. 1987; Worthey et al. 1994; Morelli et al. 2017) is affected by the intrinsic velocity dispersion that broadens the lines in the integrated spectra of the galaxies. This could render useless some weaker or close to each other indices that we defined in this work. It is therefore important to investigate how our measurements are affected by the internal kinematics of galaxies.

As done in Paper I and in order to take this into account, we broadened all the spectra by convolving them with a Gaussian of $\sigma$ varying from 115 to $400 \mathrm{~km} \mathrm{~s}^{-1}$ in steps of $25 \mathrm{~km} \mathrm{~s}^{-1}$. The indices were measured for all broadened spectra and a thirdorder polynomial fit was performed to the relative changes of each index $\Delta(\operatorname{Index}) / \operatorname{Index}\left(\sigma_{0}\right)$ as a function of the velocity dispersion. As expected for our indices, we found a strong correlation between the line strength and broadening. The indices with a low equivalent width value show a large variation even for small values of $\sigma$ whereas the strongest indices are less affected.

Figure 8 shows $\Delta(\operatorname{Index}) / \operatorname{Index}\left(\sigma_{0}\right)$ versus the broadened velocity dispersion for the indices in the $\mathrm{Y}$ atmospheric window. The faintest indices are omitted here and in the subsequent figures. The results for indices in the $\mathrm{J}, \mathrm{H}$, and $\mathrm{L}$ windows are shown in Figs. C.1-C.3, respectively.

To demonstrate the effect of broadening on the relations derived in Figs. 5, D.1, D.3, and D.6, in Fig. 9 we show, as an example, the result of a different broadening in for $\mathrm{Ti}$ and $\mathrm{Si}$ versus the effective temperature (see Sect. 7 for details). We choose $\mathrm{Ti}$ and Si since they have a small and large scatter, respectively, in their correlation with the effective temperature. As expected, as the velocity dispersion increases, the broadening tends to reduce the trends and the equivalent widths flatten for a large velocity dispersion $\left(\sigma \geq 350 \mathrm{~km} \mathrm{~s}^{-1}\right)$, especially for Ti.

All indices and their correlation with the stellar properties are of course affected by the broadening effect at a different level depending on the spectral type, because lines may suffer from cross-contamination by different species in stars with different effective temperature. Without exhaustive discussion, we point out that the most reliable and stable indices over velocity dispersion ranging up to $\sim 200-250 \mathrm{~km} \mathrm{~s}^{-1}$ are $\mathrm{CSi}, \mathrm{Ti}$, and $\mathrm{Pa} \delta$ in the $\mathrm{Y}$ atmospheric window, $\mathrm{Na}, \mathrm{FeCr}, \mathrm{K} 1, \mathrm{Si}, \mathrm{K} 2$ and $\mathrm{Pa} \beta$ in the $\mathrm{J}$ atmospheric window, $\mathrm{Mg} 2, \mathrm{Mg} 3, \mathrm{Si}, \mathrm{COMg}$, and $\mathrm{Br}_{10}$ in the $\mathrm{H}$ atmospheric window, and $\mathrm{Mg} 2$ in the $\mathrm{L}$ atmospheric window.

\section{Spectral diagnostics}

The indices defined in Sect. 5 have dependencies on the stellar properties. In this section, we describe for each atmospheric window the behavior of the most relevant features presents.

\subsection{Y atmospheric window}

Almost all indices in the $\mathrm{Y}$ atmospheric window show some dependence on $T_{\text {eff }}$, although with a different level of significance and over different temperature ranges (Fig. 5). For 
L. Morelli et al.: The Infrared Telescope Facility (IRTF) spectral library. II.
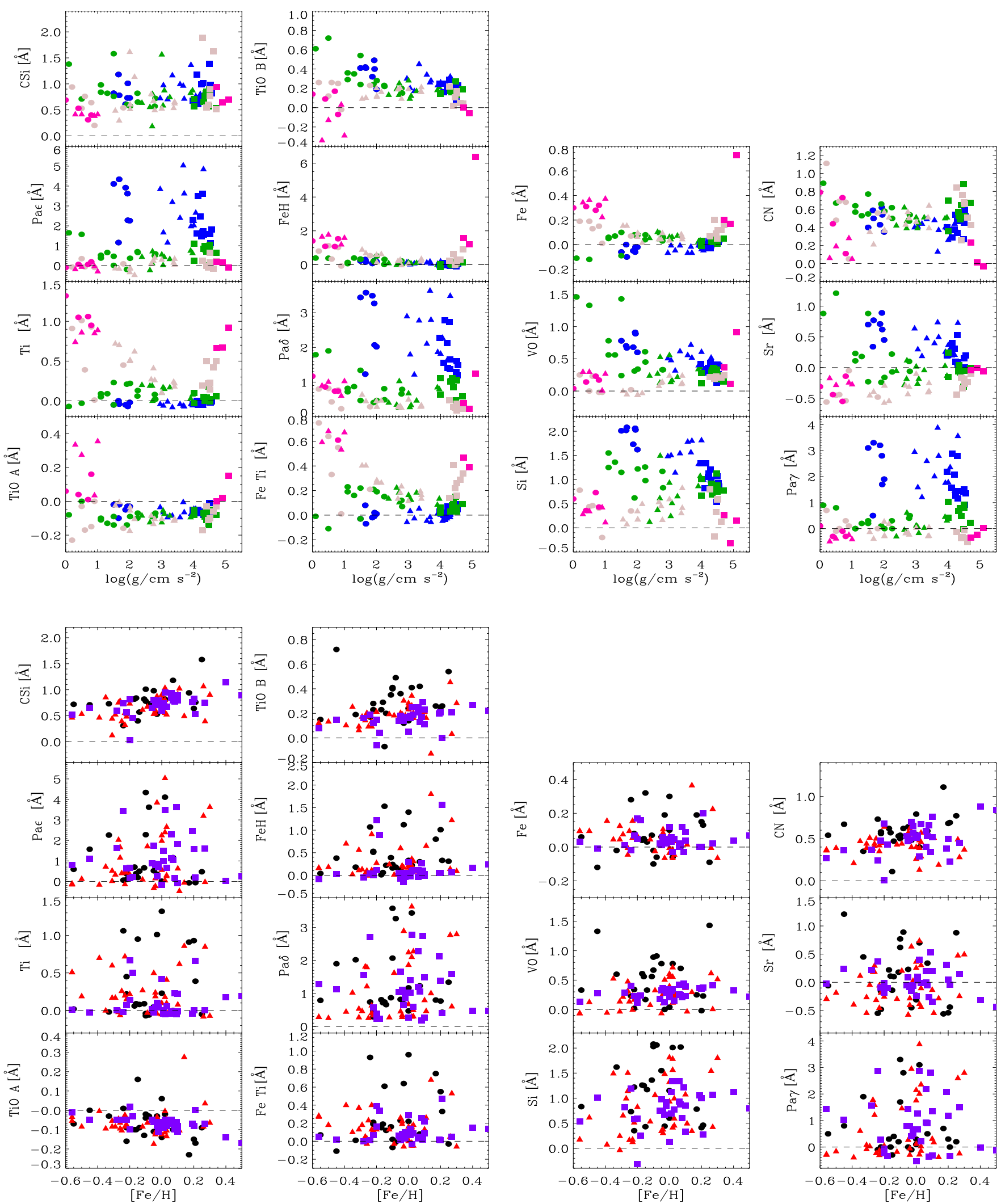

Fig. 6. Index measurements in the $\mathrm{Y}$ atmospheric window as functions of surface gravity (top panels) and metallicity (bottom panels). Symbols for supergiants, giants, and dwarf stars are as in Fig. 5. Different colors in the top panels correspond to F (blue), G (green), K (gray), and M type stars (pink), respectively.

example, $\mathrm{Pa} \epsilon, \mathrm{Pa} \delta$, and $\mathrm{Pa} \gamma$ indices are excellent temperature indicators for stars of types earlier than about G3. Other indices such as Ti, TiO A, TiO B, FeH, FeTi, Fe, and VO lines are com- plementary and work for the cooler stars. The $\mathrm{Si}, \mathrm{CN}$, and $\mathrm{Sr}$ indices are non-monotonic, which is also true to some extent for the Paschen indices. Finally, CSi has monotonic dependence 

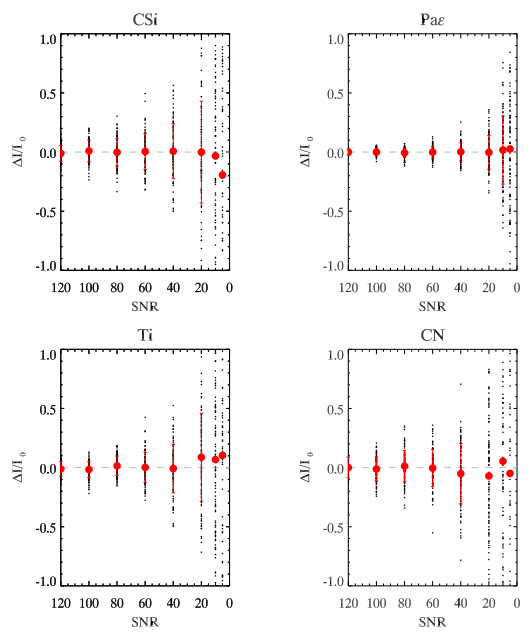

$\mathrm{FeH}$

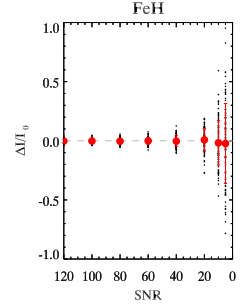

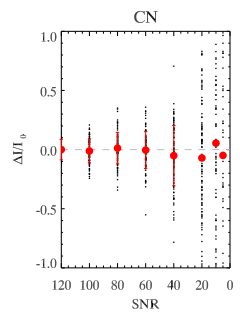

vo

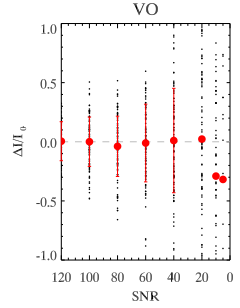

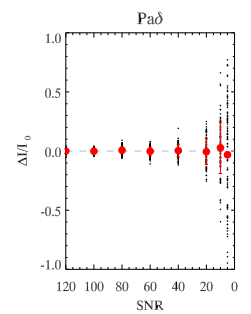

$\mathrm{Pa} \delta$
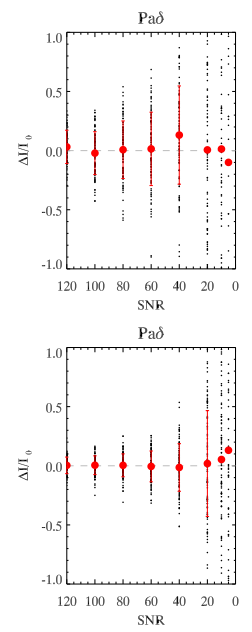

Fig. 7. Deviation of the index (measured on the noisy artificial spectrum) from the index value in the "noiseless" spectrum, normalized by the latter, plotted versus the $\mathrm{S} / \mathrm{N}$ of the noisy spectrum for $Y$ band spectral features in MI (lower panels), KIII (central panels), and FII (upper panels) stars. The $\mathrm{S} / \mathrm{N}$ is given per $\AA$.

over the almost entire range of interest, but this feature is relatively weak and shows some scatter.

The surface gravity is harder to infer from the $\mathrm{Y}$ window indices (Fig. 6, upper panels). Some molecular features related to $\mathrm{TiO}, \mathrm{FeH}, \mathrm{VO}$, and $\mathrm{CN}$ do follow monotonic trends that are not strong in comparison with the measurement errors. The trends become more evident if the samples are constrained to a given spectral type, for example, to $\mathrm{G}$ type stars for the $\mathrm{VO}$ index or to $\mathrm{M}$ stars for the FeH index. A similar effect is seen with the metallicity. Indices such as $\mathrm{CSi}$ and $\mathrm{TiO} \mathrm{B}$ show trends only when a certain luminosity class (like supergiant stars) is considered (Fig. 6, bottom panels).

\subsection{J atmospheric window}

Almost all the indices in the $\mathrm{J}$ window can serve as good $T_{\text {eff }}$ indicators (Fig. D.1). However, in most cases the trends in comparison with the scatter are relatively stronger than for the indices in the $\mathrm{Y}$ window. Notably in some cases, such as the $\mathrm{K} 1 \mathrm{~A}, \mathrm{~K} 1 \mathrm{~B}, \mathrm{~K} 2 \mathrm{~A}$, and $\mathrm{K} 2 \mathrm{~B}$ indices, the trends for stars of different luminosity classes are identical. This allows us to estimate the stellar temperature without information about the luminosity class. The dwaars cooler than mid-M type show an extremely strong $T_{\text {eff }}$ dependence for many indices, including $\mathrm{Na}, \mathrm{K} 1 \mathrm{~A}, \mathrm{C}$, $\mathrm{K} 1 \mathrm{~B}, \mathrm{Si}, \mathrm{K} 2 \mathrm{~A}$, and $\mathrm{K} 2 \mathrm{~B}$. However, this is due to contamination by molecules such as methane. These indices reach values in the dwarf stars that are not measured in the stars of other luminosity classes, making it obvious that the stars are dwarf. There are no indices that show degeneracy, with the exception of $\mathrm{Mg}$ and $\mathrm{Al}$ only for dwarf stars.

Some $\mathrm{J}$ window indices can serve as luminosity indicators. They are FeCr, K1A, K1B, and Si. Na, K2A, and Al are useful if only certain spectral types are considered (Fig. D.2, upper panels). The K1A index appears to be by far the best one, and more notably it shows the same behavior over the entire range of spectral types. Disappointingly, none of the J window indices seem to correlate with $[\mathrm{Fe} / \mathrm{H}]$, and $\mathrm{Mg}$ - and $\mathrm{Fe}$-dominated indices need further investigation (Fig. D.2, bottom panels).

\section{3. $H$ atmospheric window}

The indices in the $\mathrm{H}$ atmospheric window in general show a more complex behavior than those in the $\mathrm{Y}$ and J windows (Figs. D.3 and D.3). The dwarf stars show non-monotonic behavior more often than not and there are no indices with identical trends for supergiant, giant, and dwarf stars, except for some spectral classes, for example, $\mathrm{CO} 1, \mathrm{CO} 2, \mathrm{CO} 3, \mathrm{FeH} 1$, and $\mathrm{FeH} 2$ for stars earlier than $\mathrm{K} 0$, and $\mathrm{COMg}$ for stars earlier than $\mathrm{K} 7$. However, the different behavior of some indices in stars of different luminosity classes can be used to our advantage to separate the luminosity classes. For example, various $\mathrm{CO}$-based indices have very different values for dwarf stars, as long as they are cooler than mid-K or early-M types. This diagnostic can be brought to stars as hot as early- $\mathrm{K}$ for $\mathrm{FeH} 2$ and $\mathrm{CO} 5$ indices, and as early as $\mathrm{G}$ with the help of the $\mathrm{Mg} 1$ and $\mathrm{Mg} 2$ indices provided there are no metallicity effects. This behavior is clearly visible in Fig. D.4.

$\mathrm{Br}_{10}, \mathrm{Si}$, and $\mathrm{CO} 4$ indices are shown to be very promising $\log g$ indicators in the $\mathrm{H}$ window (Fig. D.4). None of the investigated indices here show significant metallicity trend (Fig. D.5). Unfortunately, the $\mathrm{Mg}$ feature at $\lambda \simeq 1.58 \mu \mathrm{m}$ is severely contaminated with other lines and it seems to be rather temperature sensitive at $\lambda \simeq 1.71 \mu \mathrm{m}$, (Le et al. 2011). The passband of $\mathrm{Si}$ at $\lambda \simeq 1.596 \mu \mathrm{m}$ includes the strong line of $\mathrm{Fe} \lambda \simeq 1.599 \mu \mathrm{m}$, which is also increasing with decreasing effective temperature. These effect requires further investigation but this will be possible only after widening the metallicity range of the library stars.

\subsection{L atmospheric window}

The $\mathrm{L}$ window is relatively devoid of spectral features in comparison with the other windows. We see the same variety of behavior with respect to the temperature as in the other windows (Fig. D.6). The Pf $\gamma$ index appears to be the best temperature indicator for stars earlier than G2 and various indices encompassing the $\mathrm{OH}$ band for the cooler stars. The same $\mathrm{OH}$-based indices together with the $\mathrm{SiO}$-based indices can help to separate dwarfs from giants and supergiant stars for spectral types later than K3. However, the intrinsic scatter of the relations for L window indices is larger than for the indices in the other atmospheric windows - not surprising given the stronger thermal background at $\lambda>2.5 \mu \mathrm{m}$.

The index with the most consistent behaviour with respect to the surface gravity across all the spectral types is $\mathrm{Hu}_{15}$ (Fig. D.7, upper panels), although to be reliable diagnostics the $\mathrm{S} / \mathrm{N}$ of the data is critical. Like the previous cases, we found no significant correlations with $[\mathrm{Fe} / \mathrm{H}]$ (Fig. D.7, bottom panels).

\section{Discussion and summary}

The analysis presented here is based on stellar spectra. We relate the strengths of various spectral features with the physical properties of the stars - effective temperature $T_{\text {eff }}$, surface gravity $\log g$, and metallicity $[\mathrm{Fe} / \mathrm{H}]$. However, our main goal is to relate the strengths of these spectral features to the physical parameters of stellar populations in unresolved galaxies, 


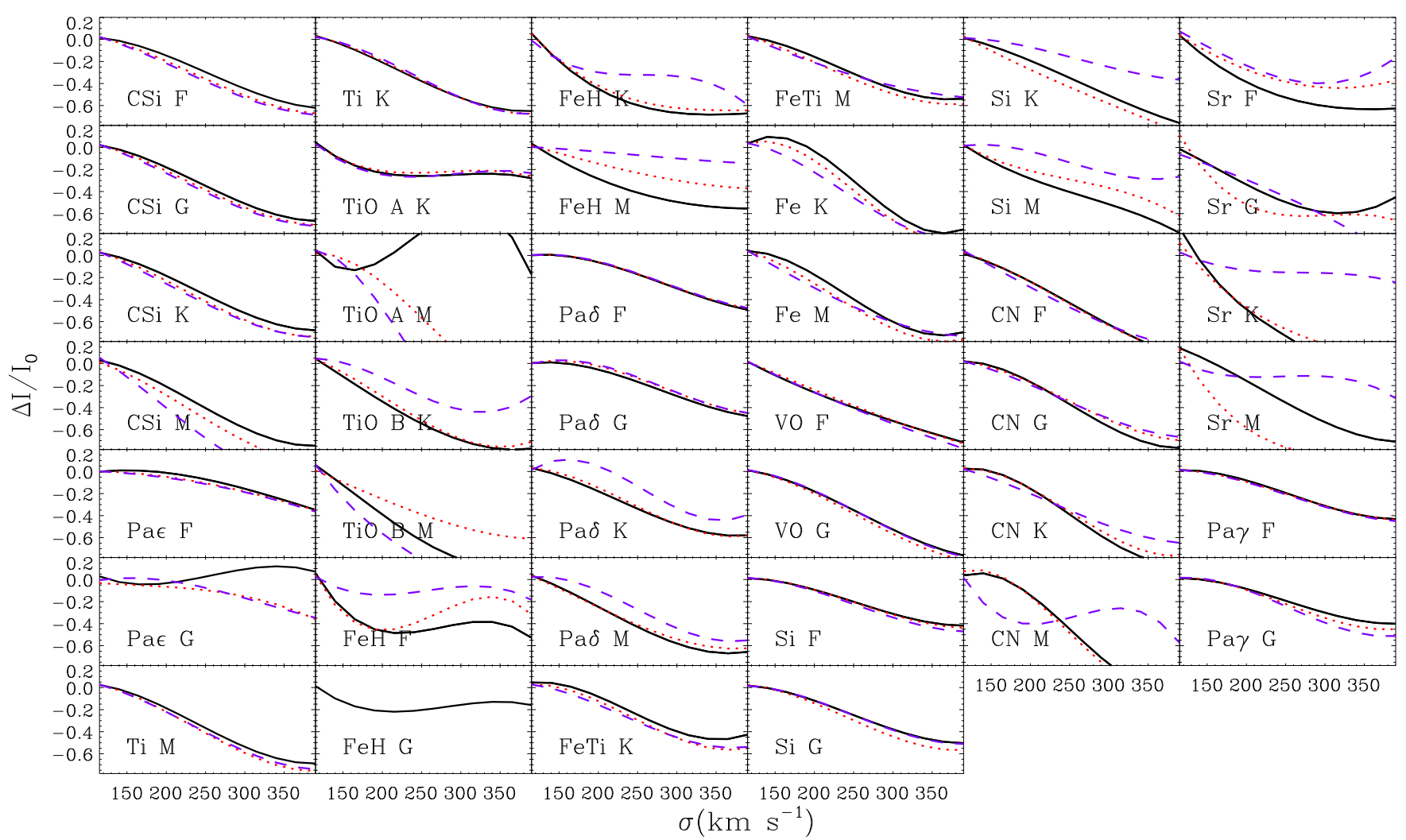

Fig. 8. Sensitivity of the indices in the $\mathrm{Y}$ atmospheric window to the velocity dispersion broadening. The relative variations of indices $\Delta(\operatorname{Index}) / \operatorname{Index}\left(\sigma_{0}\right)$ for different luminosity classes and spectral types are shown: supergiants with a black solid line, giants with a red dotted line, and dwarf stars with a violet dashed line.
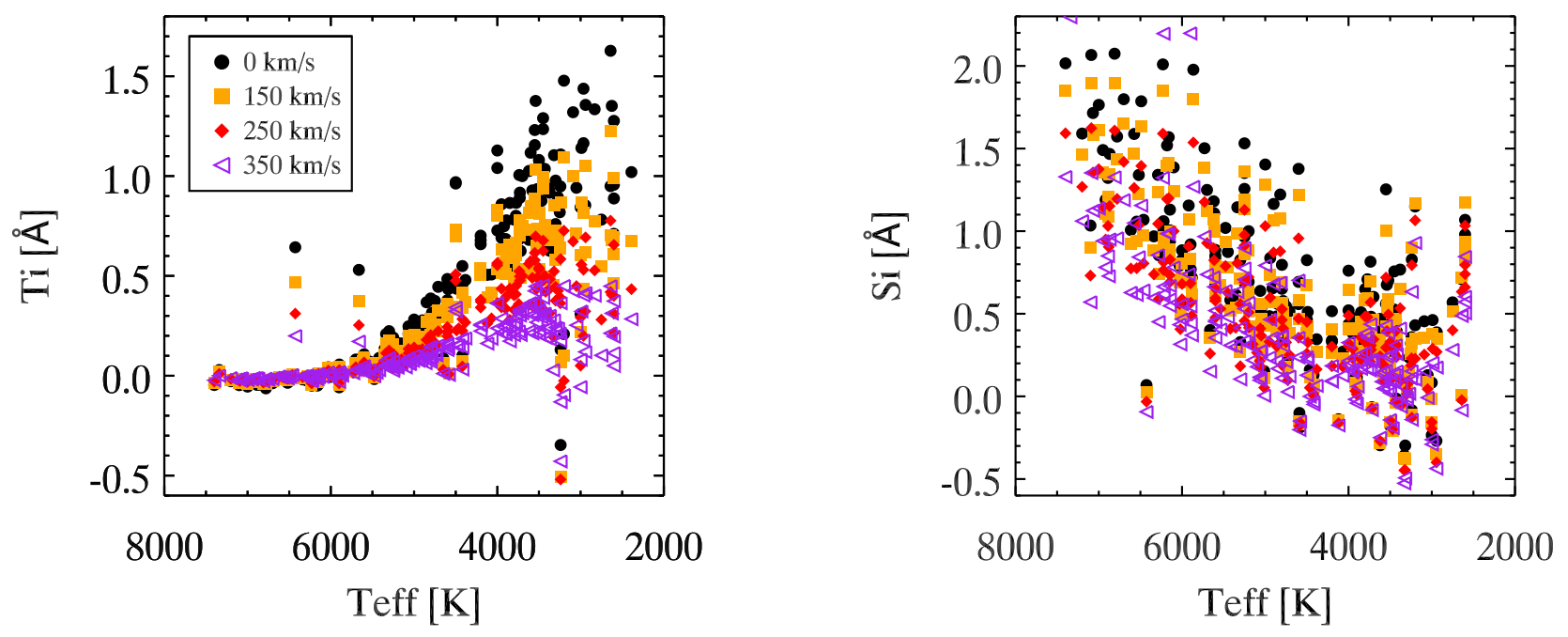

Fig. 9. Effect of the velocity dispersion broadening on the Ti- $T_{\text {eff }}$ (left panel) and $\mathrm{Si}-T_{\text {eff }}$ (right panel). With a black circle we show the original relation, and with a yellow square, red diamonds, and violet triangle the relation after a broadening of 150,250 , and $350 \mathrm{~km} \mathrm{~s}^{-1}$, respectively.

and we adopt a simple and straightforward mapping of one to the other. The temperature $T_{\text {eff }}$ is related to the population age, because the luminosity-weighted temperature decreases as the population passively evolves from being dominated by younger main-sequence stars towards older red giant stars. The surface gravity $\log g$ tells us what is the dominant luminosity class of a population. Finally, the metallicity $[\mathrm{Fe} / \mathrm{H}]$ translates into the cumulative abundance of the dominant population in a galaxy. We will investigate these diagnostics in a forthcoming paper (Gasparri et al. 2020) by measuring them in the sample of galax- ies observed with X-shooter (François et al. 2019). Furthermore, we will also compare the observational behavior of our defined indices with the predictions from the theoretical models (e.g., Röck et al. 2016; Conroy et al. 2018).

We point out that the diagnostics described here can also be used for spectral typing of individual stars. Last but not least, we stress that the behavior of the indices is not necessarily the same as the behavior of the particular species that dominate these indices, because of the possible contamination by other species as noted also by Conroy \& van Dokkum (2012) in their models 
Table 2. Summary of the best indicators for the different stellar physical parameters.

\begin{tabular}{|c|c|c|c|}
\hline $\begin{array}{l}\text { Stellar parameters } \\
\text { (1) }\end{array}$ & $\begin{array}{l}\text { Index } \\
(2)\end{array}$ & $\begin{array}{l}\text { Quality } \\
\text { (3) }\end{array}$ & $\begin{array}{c}\text { Notes } \\
(4)\end{array}$ \\
\hline \multicolumn{4}{|c|}{ Y atmospheric windows } \\
\hline \multirow{3}{*}{$T_{\mathrm{eff}}$} & $\operatorname{Pa} \epsilon, \operatorname{Pa} \delta, \operatorname{Pa} \gamma$ & $\mathrm{S}$ & F, G stars \\
\hline & Ti,TiO A,TiO B,FeH,FeTi,Fe, VO & $\mathrm{S}$ & $\mathrm{G}, \mathrm{K}, \mathrm{M}$ stars \\
\hline & CSi & $\mathrm{W}$ & Stars of all spectral type \\
\hline $\log g$ & $\mathrm{TiO}, \mathrm{FeH}, \mathrm{VO}, \mathrm{CN}$ & $\mathrm{S} / \mathrm{W}$ & Very dependent on star spectral type \\
\hline \multirow[t]{2}{*}[\mathrm{Fe}/\mathrm{H}]{} & $\mathrm{TiO} \mathrm{B}$ & $\mathrm{S}$ & Only in supergiant stars \\
\hline & \multicolumn{3}{|c|}{$\mathrm{J}$ atmospheric windows } \\
\hline \multirow[t]{2}{*}{$T_{\mathrm{eff}}$} & $\mathrm{K} 1 \mathrm{~B}, \mathrm{~K} 2 \mathrm{~A}, \mathrm{~K} 2 \mathrm{~B}$ & $\mathrm{~S}$ & Similar trend in stars of all spectral types \\
\hline & $\mathrm{K} 1 \mathrm{~A}, \mathrm{Na}, \mathrm{Si}$ & S & M stars, methane contamination \\
\hline \multirow[t]{3}{*}{$\log g$} & $\mathrm{FeCr}, \mathrm{K} 1 \mathrm{~B}, \mathrm{Na}, \mathrm{Si}, \mathrm{Al}$ & S & Dependent on star spectral type \\
\hline & $\mathrm{K} 1 \mathrm{~A}$ & $\mathrm{~S}$ & All spectral type stars \\
\hline & \multicolumn{3}{|c|}{$\mathrm{H}$ atmospheric windows } \\
\hline$T_{\text {eff }}$ & $\mathrm{CO} 1, \mathrm{CO} 2, \mathrm{CO} 3, \mathrm{FeH} 1, \mathrm{FeH} 2, \mathrm{COMg}$ & $\mathrm{S}$ & Mostly in F, G stars \\
\hline $\log g$ & $\mathrm{Br}_{10}, \mathrm{Si}, \mathrm{CO} 4$ & $\mathrm{~S}$ & Stars of all spectral types \\
\hline \multicolumn{4}{|c|}{ L atmospheric windows } \\
\hline$T_{\text {eff }}$ & Pf $\gamma$ & W & In stars earlier than G2 \\
\hline $\log g$ & $\mathrm{Mg} 3, \mathrm{Hu}_{15}$ & W & Stars of all spectral type \\
\hline
\end{tabular}

Notes. For the details, please refer to Sect. 7. The columns show the following: (1) stellar physical parameters (2) spectral feature (3) quality of the index: strong $(\mathrm{S})$ or weak $(\mathrm{W})(4)$ : notes on the index.

analyzing the initial mass function indicators. For example, some hydrogen and metal lines are contaminated by molecular bands in the late-type giant and supergiant stars.

To summarize, we present here an analysis of stellar spectra in the $\mathrm{Y}, \mathrm{J}, \mathrm{H}$, and $\mathrm{L}$ atmospheric windows, based on high-quality medium resolution $(R \sim 2000-2500)$ spectra of more than 200 stars with almost solar metallicity $(-0.5 \leq[\mathrm{Fe} / \mathrm{H}] \leq 0.5 \mathrm{dex})$ and a spectral S/N of about 100 (Rayner et al. 2009). We identify the best indicators for the different stellar physical parameters, and as discussed above, of some integrated parameters of the stellar populations in unresolved galaxies. The results discussed in Sect. 7 have been summarized in Table 2.

Features sensitive to the effective temperature are present and measurable in all the investigated atmospheric windows. The surface gravity is more challenging: the $\mathrm{H}$ window offers better tools for a separation between dwarf and giant stars, followed by the $\mathrm{J}$ window. This is almost impossible in the $\mathrm{Y}$ and $\mathrm{L}$ windows. The diagnostics in the $\mathrm{L}$ window are the most challenging because the spectra are typically noisier than in other windows due to the higher thermal background emission. However, the next generation of both extremely large ground-based telescopes and space-based facilities will work in this regime, making even these $\mathrm{L}$ window diagnostic tools quite valuable.

Acknowledgements. We thank the anonymous referee for the useful discussion and suggestions. We have made extensive use of the SIMBAD Database at CDS (Center de Données astronomiques) Strasbourg, the NASA/IPAC Extragalactic Database (NED), which is operated by the Jet Propulsion Laboratory, CalTech, under contract with NASA, and of the VizieR catalog access tool, CDS, Strasbourg, France. EMC, AP, EDB, and LM are supported by Padua University through grants DOR1715817/17, DOR1885254/18, DOR1935272/19, and BIRD164402/16. EMC, AP, and EDB are also supported by MIUR grant PRIN 2017 20173ML3WW_001.

\section{References}

Arentsen, A., Prugniel, P., Gonneau, A., et al. 2019, A\&A, 627, A138 Blum, R. D., Ramond, T. M., Conti, P. S., Figer, D. F., \& Sellgren, K. 1997, AJ, 113,1855
Cesetti, M., Pizzella, A., Ivanov, V. D., et al. 2013, A\&A, 549, A129

Chen, Y., Trager, S., Peletier, R., \& Lançon, A. 2011, J. Phys. Conf. Ser., 328, 012023

Chen, Y.-P., Trager, S. C., Peletier, R. F., et al. 2014, A\&A, 565, A117

Cirasuolo, M., Afonso, J., Bender, R., et al. 2011, The Messenger, 145, 11

Conroy, C., \& van Dokkum, P. G. 2012, ApJ, 760, 71

Conroy, C., Villaume, A., van Dokkum, P. G., \& Lind, K. 2018, ApJ, 854, 139

Cooper, H. D. B., Lumsden, S. L., Oudmaijer, R. D., et al. 2013, MNRAS, 430, 1125

Cuby, J. G., Morris, S., Fusco, T., et al. 2010, in Ground-based and Airborne Instrumentation for Astronomy III, Proc. SPIE, 7735, 77352D

Cunha, K., Sellgren, K., Smith, V. V., et al. 2007, ApJ, 669, 1011

Cushing, M. C., Rayner, J. T., Davis, S. P., \& Vacca, W. D. 2003, ApJ, 582, 1066

Cushing, M. C., Rayner, J. T., \& Vacca, W. D. 2005, ApJ, 623, 1115

Dallier, R., Boisson, C., \& Joly, M. 1996, A\&AS, 116, 239

Davies, B., Origlia, L., Kudritzki, R.-P., et al. 2009a, ApJ, 694, 46

Davies, B., Origlia, L., Kudritzki, R.-P., et al. 2009b, ApJ, 696, 2014

Dressler, A., Lynden-Bell, D., Burstein, D., et al. 1987, ApJ, 313, 42

Engelbracht, C. W., Rieke, M. J., Rieke, G. H., Kelly, D. M., \& Achtermann, J. M. 1998, ApJ, 505, 639

Evans, C. J., Davies, B., Kudritzki, R.-P., et al. 2011, A\&A, 527, A50

Fellgett, P. B. 1951, MNRAS, 111, 537

Fernández-Trincado, J. G., Beers, T. C., Placco, V. M., et al. 2019, ApJ, 886, L8 François, P., Morelli, L., Pizzella, A., et al. 2019, A\&A, 621, A60

Gasparri, D., Morelli, L., Ivanov, V. D., et al. 2020, A\&A, submitted

García Pérez, A. E., Allende Prieto, C., Holtzman, J. A., et al. 2016, AJ, 151, 144

Ghosh, S., Mondal, S., Das, R., \& Khata, D. 2019, MNRAS, 484, 4619

Gonneau, A., Lyubenova, M., Lançon, A., et al. 2020, A\&A, 634, A133

Hanson, M. M., Kudritzki, R. P., Kenworthy, M. A., Puls, J., \& Tokunaga, A. T. 2005, ApJS, 161, 154

Heras, A. M., Shipman, R. F., Price, S. D., et al. 2002, A\&A, 394, 539

Hillenbrand, L. A., Foster, J. B., Persson, S. E., \& Matthews, K. 2002, PASP, 114,708

Huynh, A. N. L., Kang, W., Pak, S., et al. 2011, J. Korean Astron. Soc., 44, 125

Ivanov, V. D., Rieke, G. H., Groppi, C. E., et al. 2000, ApJ, 545, 190

Ivanov, V. D., Rieke, M. J., Engelbracht, C. W., et al. 2004, ApJS, 151, 387

Johnson, H. L. 1966, ARA\&A, 4, 193

Johnson, H. L., \& Méndez, M. E. 1970, AJ, 75, 785

Joyce, R. R., Hinkle, K. H., Wallace, L., Dulick, M., \& Lambert, D. L. 1998, AJ, 116,2520

Lancon, A., \& Rocca-Volmerange, B. 1992, A\&AS, 96, 593

Lançon, A., \& Wood, P. R. 2000, A\&AS, 146, 217

Lançon, A., Hauschildt, P. H., Ladjal, D., \& Mouhcine, M. 2007, A\&A, 468, 205

Le, H. A. N., Kang, W., Pak, S., et al. 2011, ArXiv e-prints [arXiv:1108. 1499]

Lebzelter, T., Seifahrt, A., Uttenthaler, S., et al. 2012, A\&A, 539, A109 
L. Morelli et al.: The Infrared Telescope Facility (IRTF) spectral library. II.

Leggett, S. K., Allard, F., Berriman, G., Dahn, C. C., \& Hauschildt, P. H. 1996, ApJS, 104, 117

Lodieu, N., Scholz, R.-D., McCaughrean, M. J., et al. 2005, A\&A, 440, 1061 Lyubchik, Y., Jones, H. R. A., Pavlenko, Y. V., et al. 2004, A\&A, 416, 655

McKellar, A. 1988, PASP, 100, 1191

McLean, I. S., McGovern, M. R., Burgasser, A. J., et al. 2003, ApJ, 596, 561 Mennickent, R. E., Sabogal, B., Granada, A., \& Cidale, L. 2009, PASP, 121, 125

Merrill, K. M., \& Ridgway, S. T. 1979, ARA\&A, 17, 9

Meyer, M. R., Edwards, S., Hinkle, K. H., \& Strom, S. E. 1998, ApJ, 508, 397

Mobasher, B., Crampton, D., \& Simard, L. 2010, in Ground-based and Airborne Instrumentation for Astronomy III, Proc. SPIE, 7735, 77355P

Morelli, L., Pizzella, A., Coccato, L., et al. 2017, A\&A, 600, A76

Morris, P. W., Eenens, P. R. J., Hanson, M. M., Conti, P. S., \& Blum, R. D. 1996, ApJ, 470, 597

Nicholls, C. P., Lebzelter, T., Smette, A., et al. 2017, A\&A, 598, A79

Origlia, L., \& Rich, R. M. 2004, AJ, 127, 3422

Origlia, L., Moorwood, A. F. M., \& Oliva, E. 1993, A\&A, 280, 536

Origlia, L., Rich, R. M., \& Castro, S. 2002, AJ, 123, 1559

Origlia, L., Ferraro, F. R., Bellazzini, M., \& Pancino, E. 2003, ApJ, 591, 916

Park, S., Lee, J.-E., Kang, W., et al. 2018, ApJS, 238, 29

Ranade, A., Gupta, R., Ashok, N. M., \& Singh, H. P. 2004, Bull. Astron. Soc. India, 32, 311

Ranade, A. C., Ashok, N. M., Singh, H. P., \& Gupta, R. 2007, Bull. Astron. Soc India, 35, 359
Rayner, J. T., Cushing, M. C., \& Vacca, W. D. 2009, ApJS, 185, 289

Ridgway, S. T., Carbon, D. F., Hall, D. N. B., \& Jewell, J. 1984, ApJS, 54, 177

Riffel, R., Rodríguez-Ardila, A., Brotherton, M. S., et al. 2019, MNRAS, 486, 3228

Röck, B., Vazdekis, A., Peletier, R. F., Knapen, J. H., \& Falcón-Barroso, J. 2015, MNRAS, 449, 2853

Röck, B., Vazdekis, A., Ricciardelli, E., et al. 2016, A\&A, 589, A73

Schinnerer, E., Eckart, A., Quirrenbach, A., et al. 1997, ApJ, 488, 174

Sharon, C., Hillenbrand, L., Fischer, W., \& Edwards, S. 2010, AJ, 139, 646

Shimonishi, T., Onaka, T., Kato, D., et al. 2013, AJ, 145, 32

Sloan, G. C., Kraemer, K. E., Price, S. D., \& Shipman, R. F. 2003, ApJS, 147, 379

Smith, V. V., Cunha, K., Shetrone, M. D., et al. 2013, ApJ, 765, 16

Vandenbussche, B., Beintema, D., de Graauw, T., et al. 2002, A\&A, 390, 1033

Vazdekis, A., Koleva, M., Ricciardelli, E., Röck, B., \& Falcón-Barroso, J. 2016, MNRAS, 463, 3409

Venkata Raman, V., \& Anandarao, B. G. 2008, MNRAS, 385, 1076

Villaume, A., Conroy, C., Johnson, B., et al. 2017, ApJS, 230, 23

Wallace, L., \& Hinkle, K. 2002, AJ, 124, 3393

Wallace, L., Meyer, M. R., Hinkle, K., \& Edwards, S. 2000, ApJ, 535, 325

Wing, R. F., \& Spinrad, H. 1970, ApJ, 159, 973

Worthey, G., Faber, S. M., Gonzalez, J. J., \& Burstein, D. 1994, ApJS, 94, 687 


\section{Appendix A: Main spectral features plots}

The model spectra in $\mathrm{Y}, \mathrm{J}, \mathrm{H}$ and $\mathrm{L}$ atmospheric windows for stars of different luminosity classes are shown in Figs. 1 and A.1-A.11.

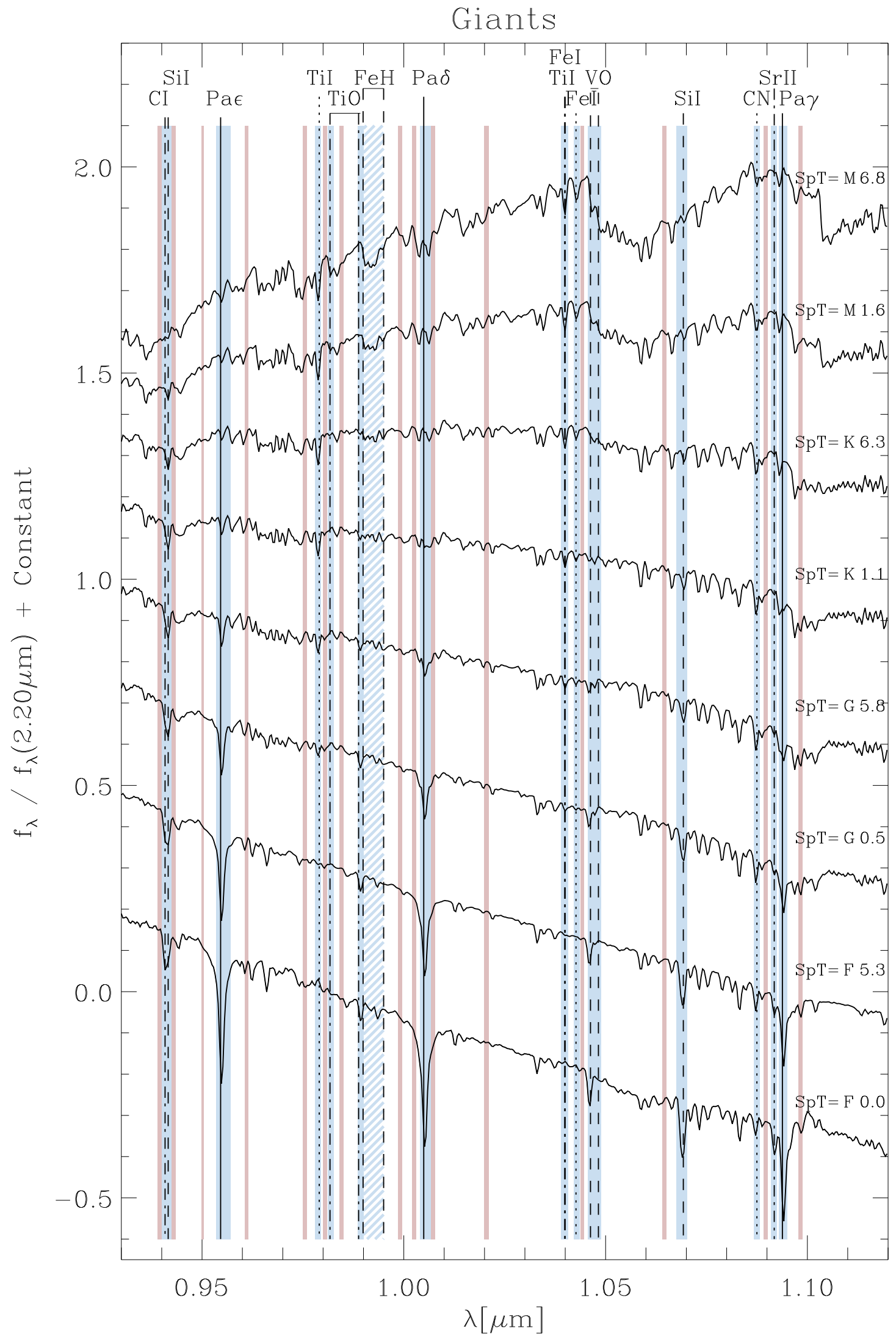

Fig. A.1. Y atmospheric window model spectrum of giants. Figure 1 gives details. 
L. Morelli et al.: The Infrared Telescope Facility (IRTF) spectral library. II.

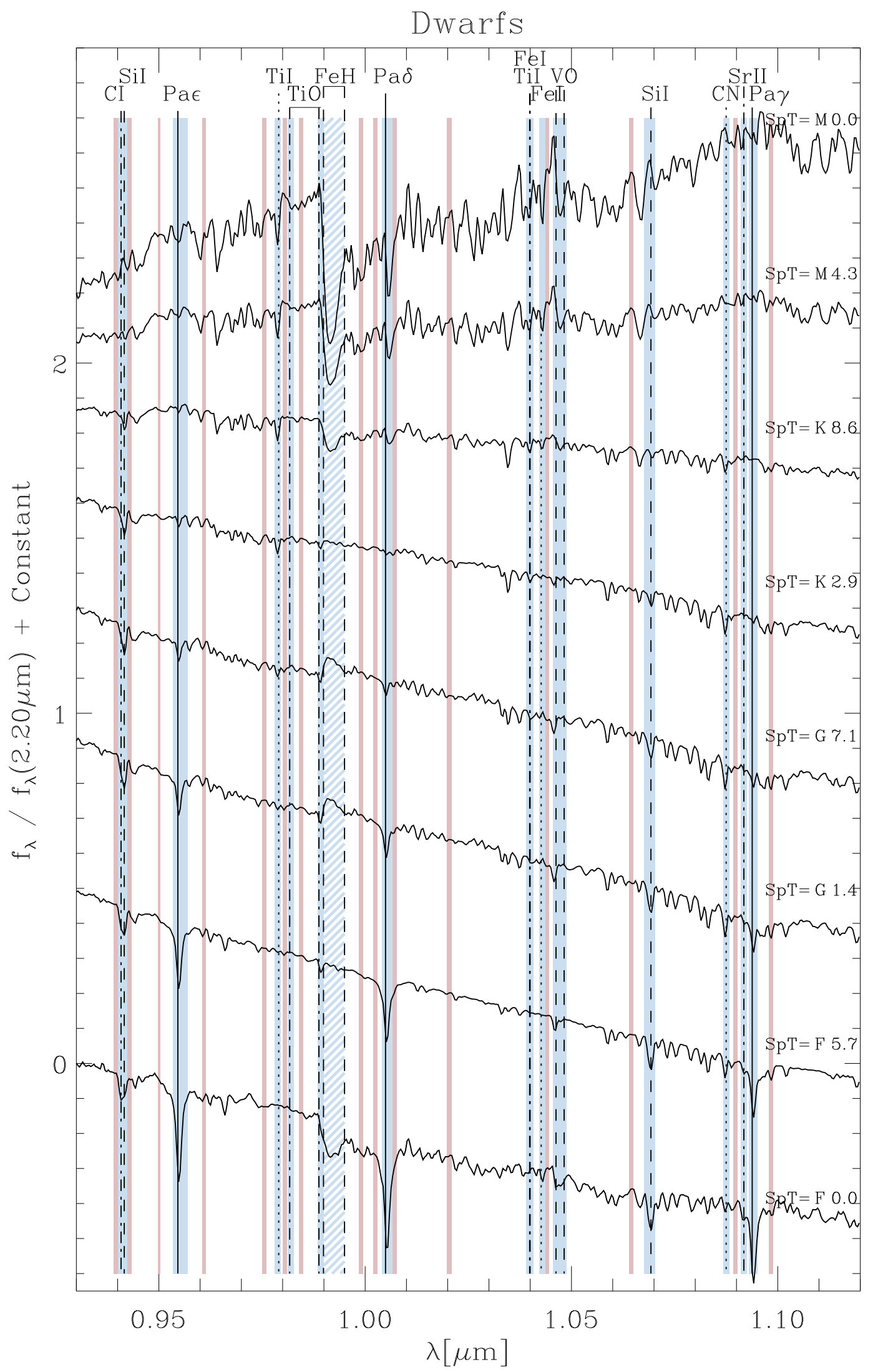

Fig. A.2. Y atmospheric window model spectrum of dwarfs. Figure 1 gives details. 
Supergiants

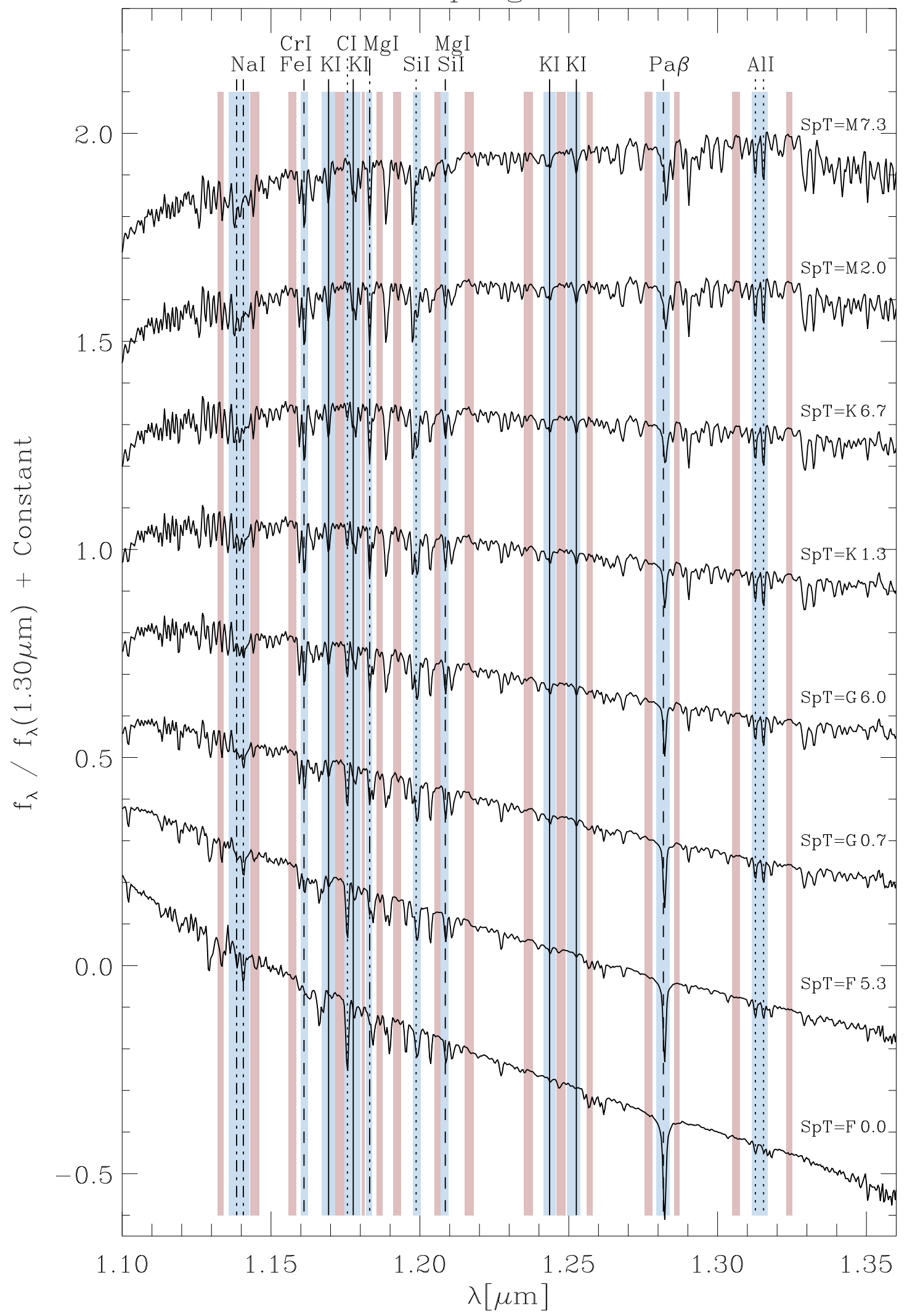

Fig. A.3. J atmospheric window model spectrum of supergiants. The flux-normalization here is performed at $1.30 \mu \mathrm{m}$. Figure 1 gives details. 
L. Morelli et al.: The Infrared Telescope Facility (IRTF) spectral library. II.

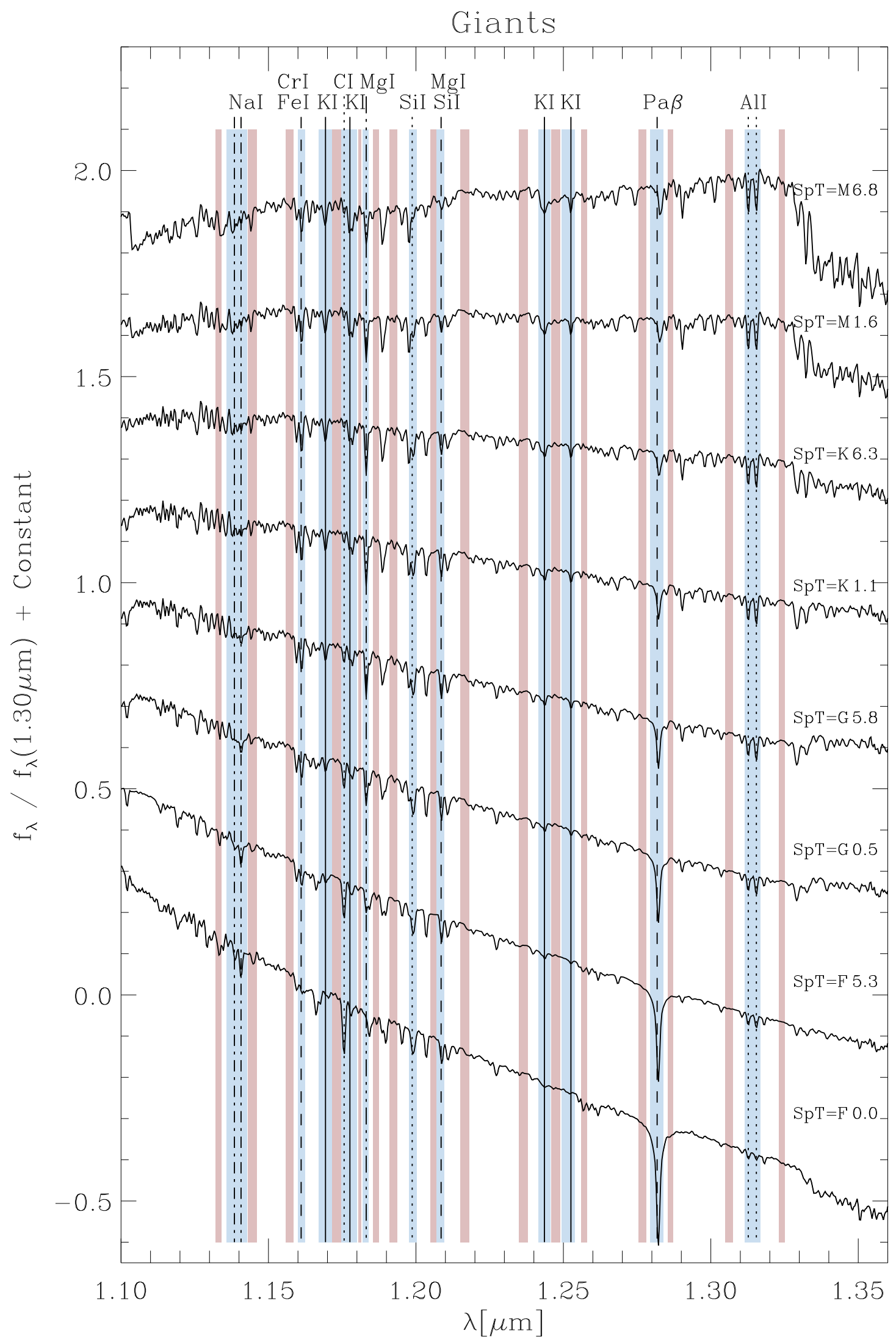

Fig. A.4. J atmospheric window model spectrum of giants. Figure A.3 gives details. 


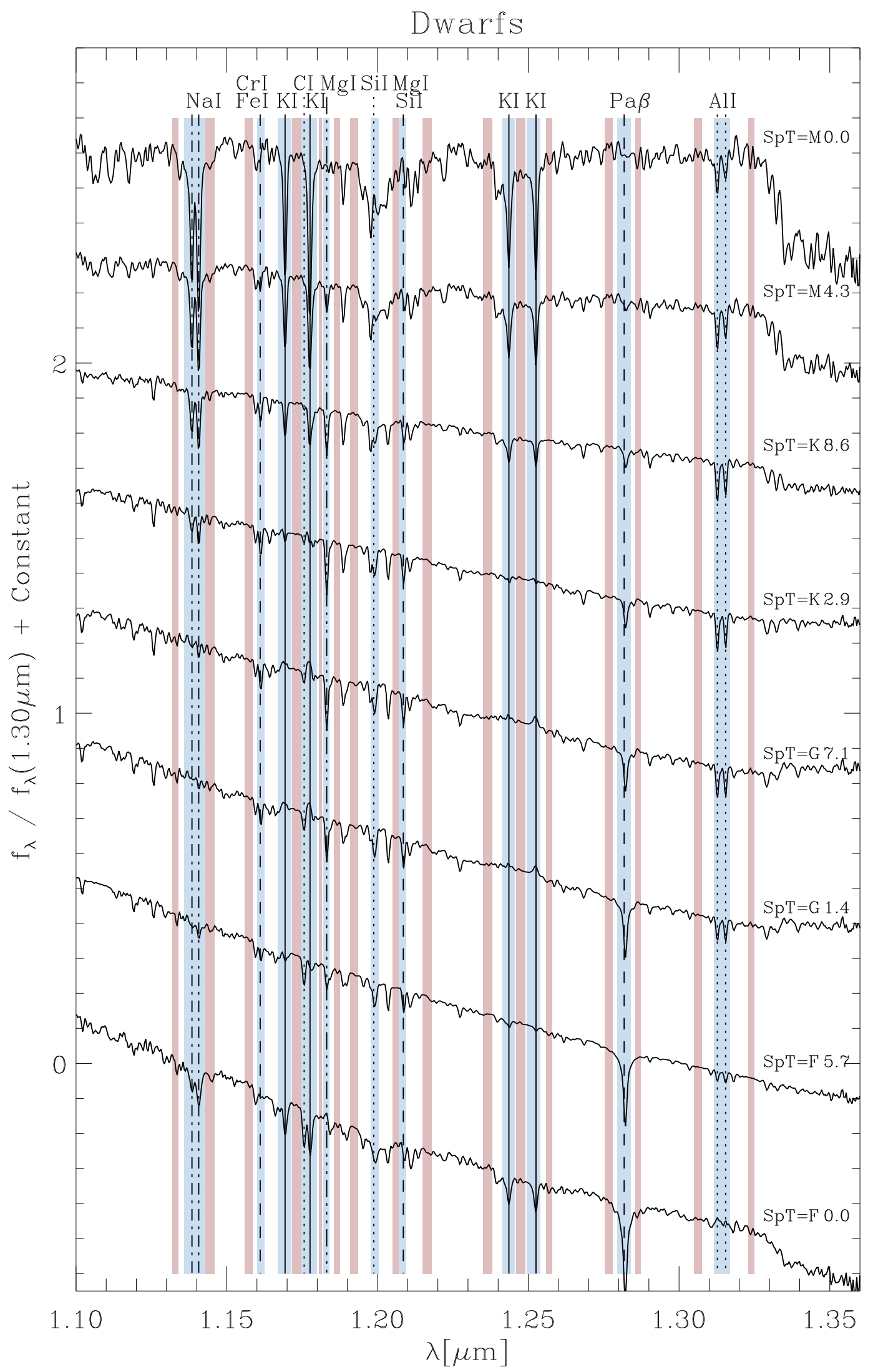

Fig. A.5. J atmospheric window model spectrum of dwarfs. Figure A.3 gives details. 


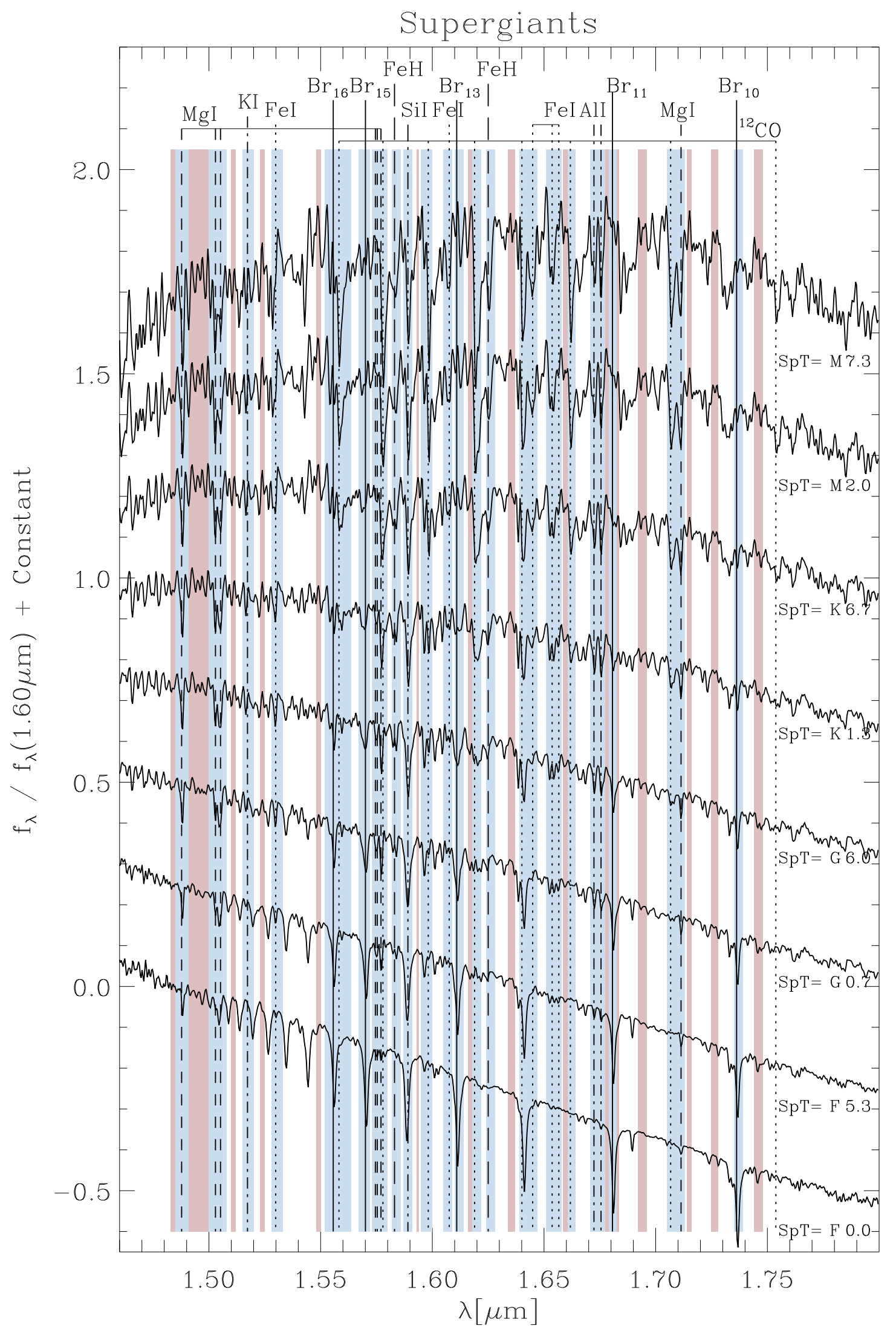

Fig. A.6. H window model spectrum of supergiants. The flux-normalization here is performed at $1.60 \mu \mathrm{m}$. Figure 1 gives details. 


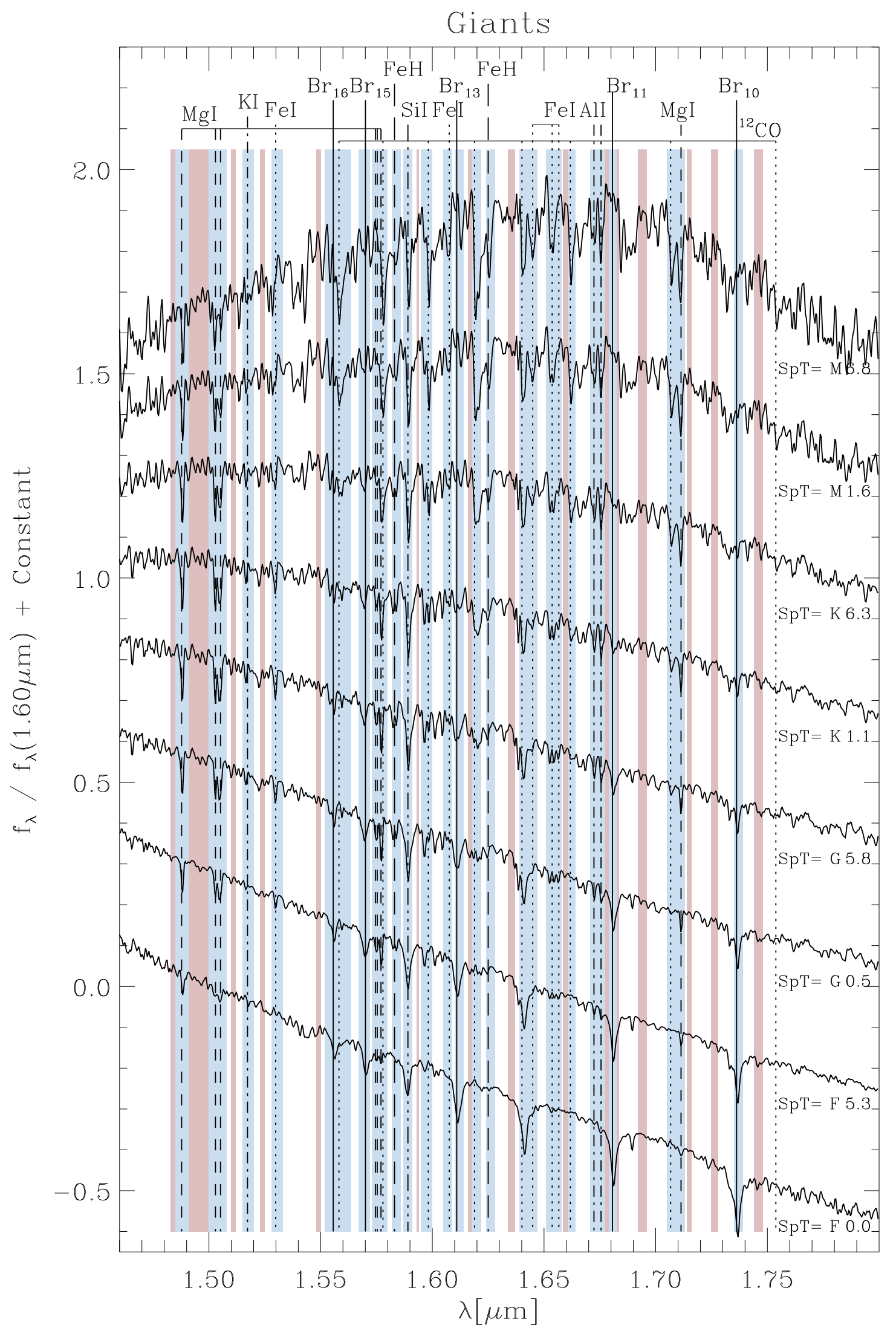

Fig. A.7. H window model spectrum of giants. Figure A.6 gives details. 
L. Morelli et al.: The Infrared Telescope Facility (IRTF) spectral library. II.

Dwarfs

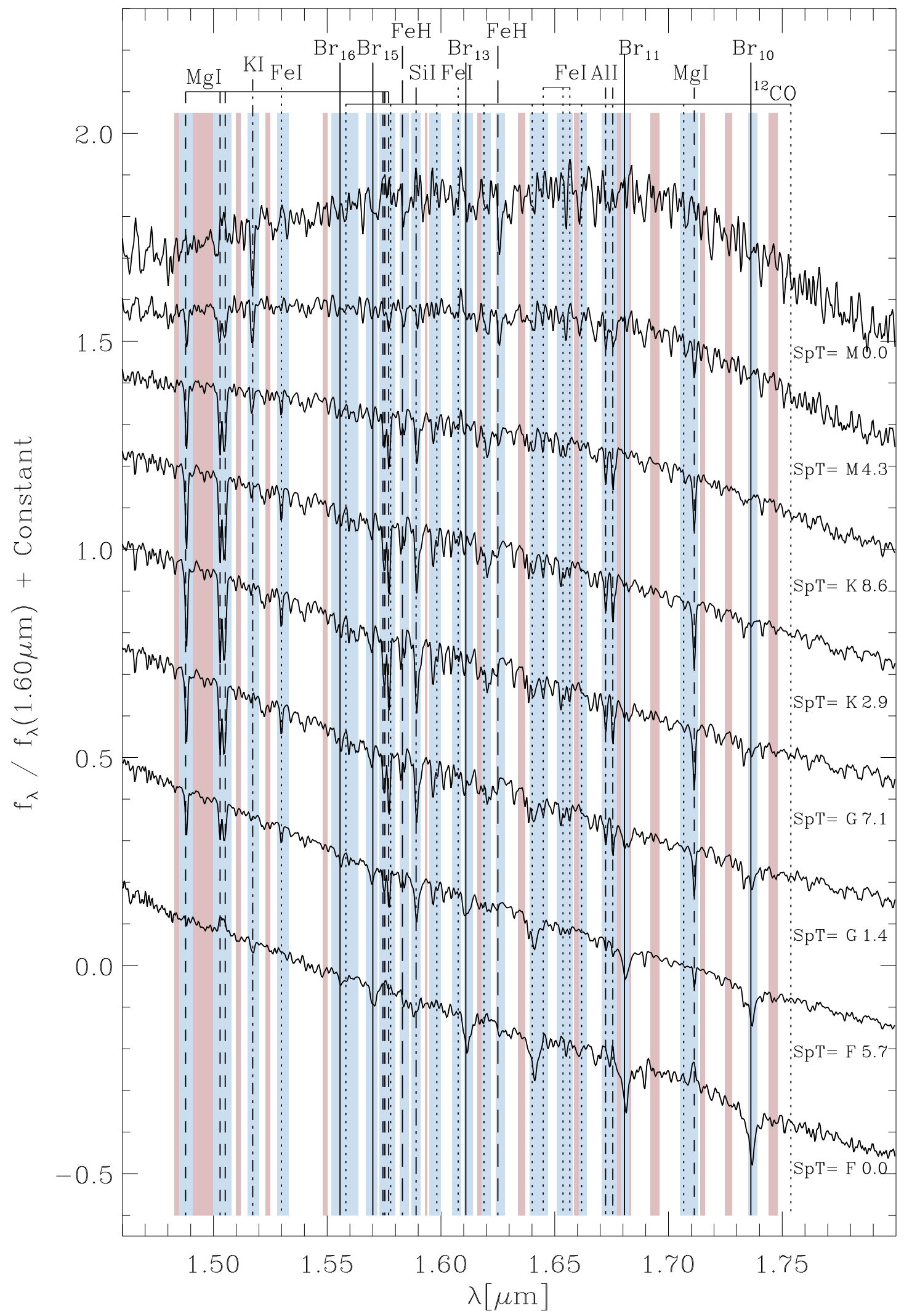

Fig. A.8. $\mathrm{H}$ window model spectrum of dwarfs. Figure A.6 gives details. 
Supergiants

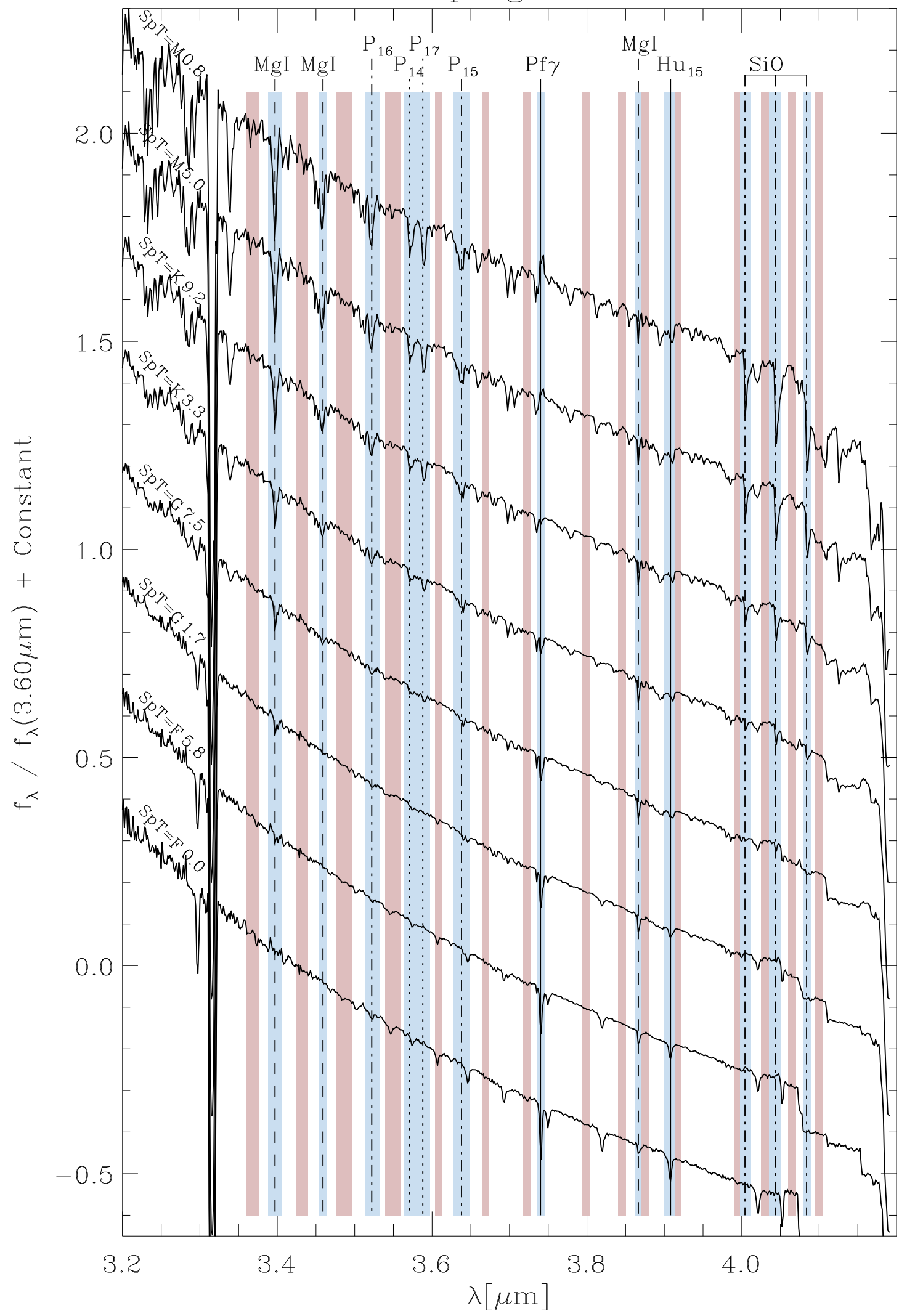

Fig. A.9. $\mathrm{L}$ atmospheric window model spectrum of supergiants. The flux-normalization here is performed at $3.60 \mu \mathrm{m}$. Figure 1 gives details. 
L. Morelli et al.: The Infrared Telescope Facility (IRTF) spectral library. II.

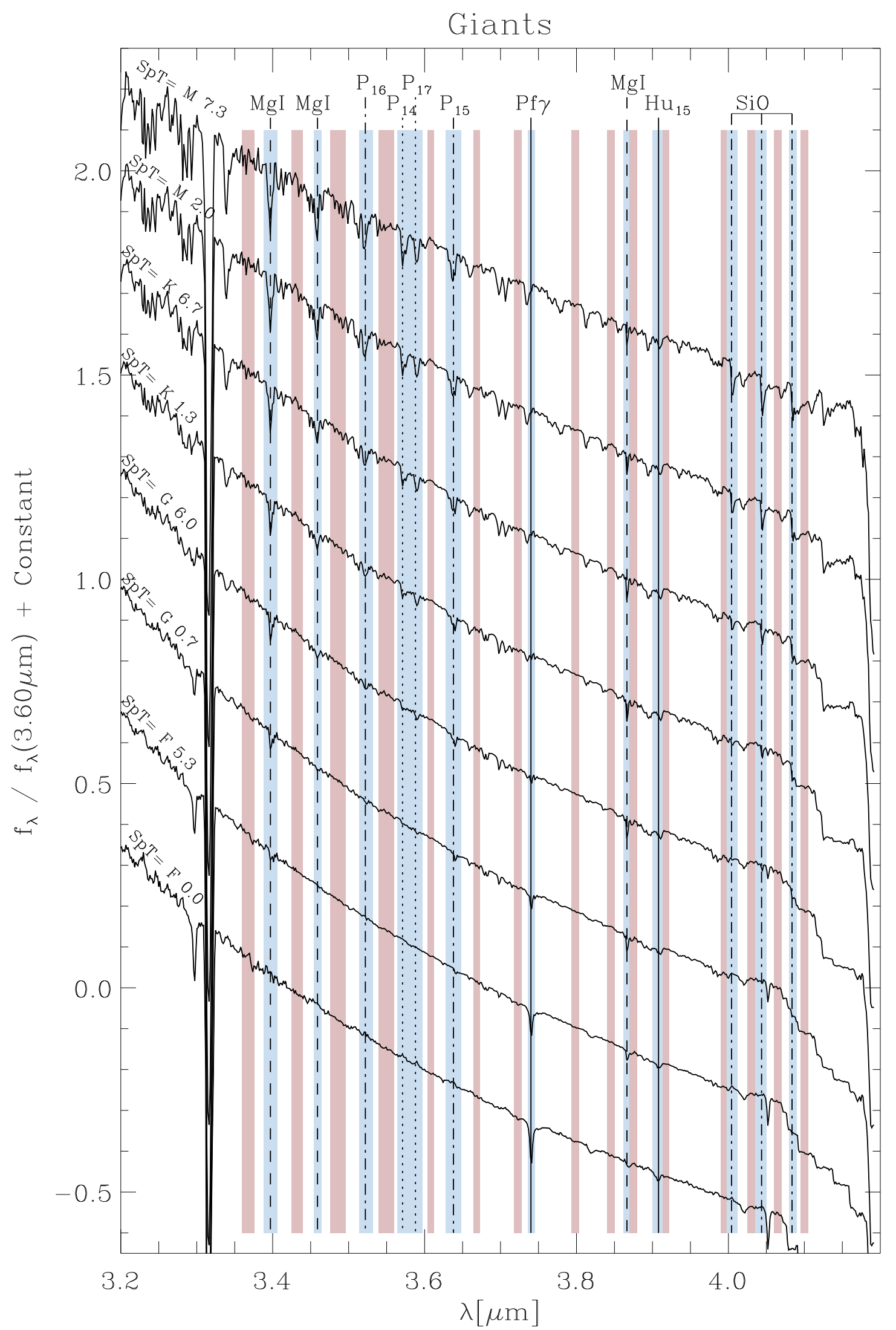

Fig. A.10. L atmospheric window model spectrum of giants. Figure A.9 gives details. 


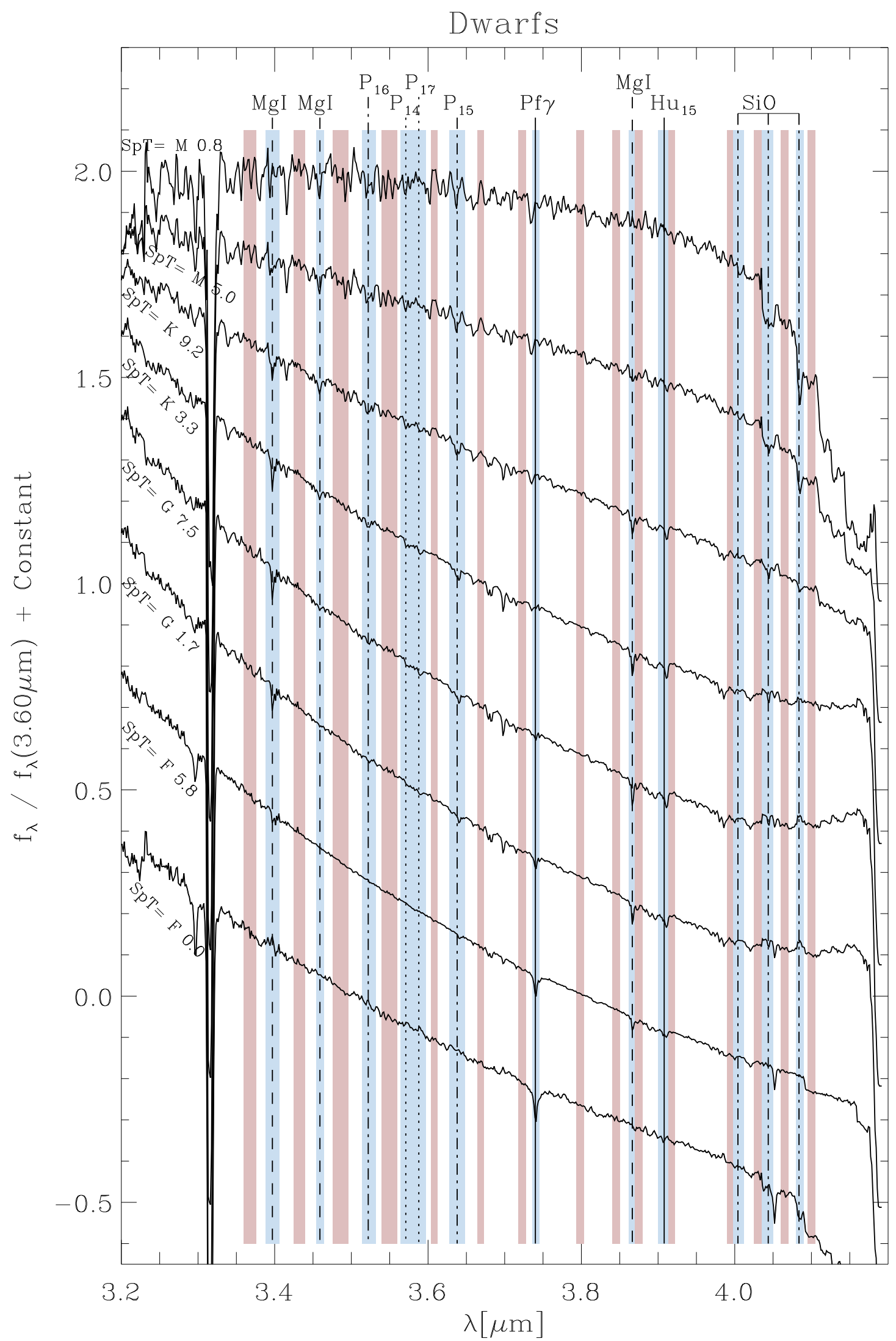

Fig. A.11. $\mathrm{L}$ atmospheric window model spectrum of dwarfs. Figure A.9 gives details. 
L. Morelli et al.: The Infrared Telescope Facility (IRTF) spectral library. II.

\section{Appendix B: Sensitivity map plots}

The sensitivity maps for SpT in $\mathrm{J}, \mathrm{H}$, and L atmospheric windows for stars of different luminosity classes are shown in Figs. 2 and B.1-A.11. The corresponding sensitivity maps for surface gravity in $\mathrm{Y}, \mathrm{J}, \mathrm{H}$, and $\mathrm{L}$ atmospheric windows for stars of different luminosity classes are shown in Figs. 2 and B.1-A.11.

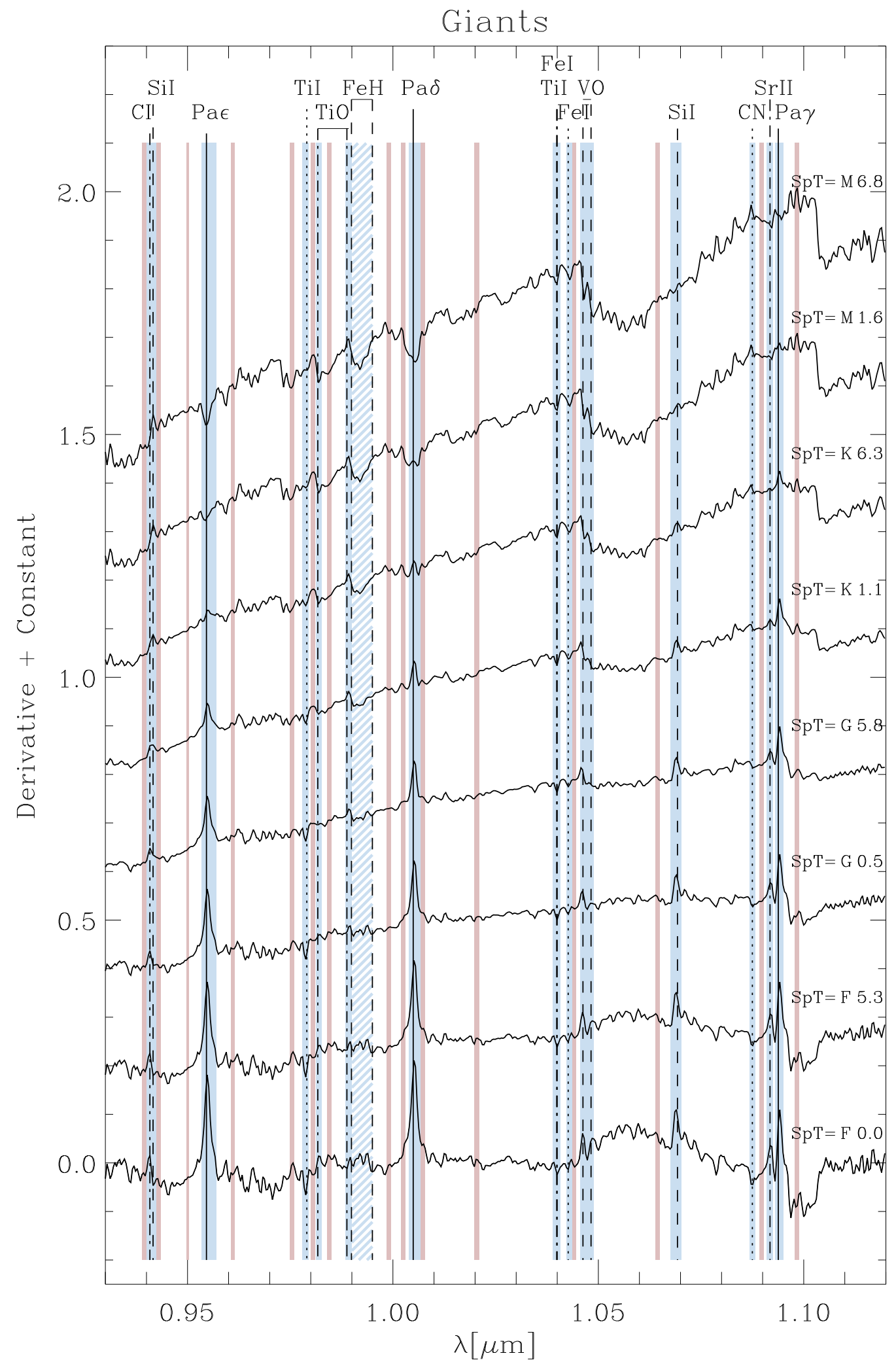

Fig. B.1. Y atmospheric window sensitivity map for SpT of giants. Figure 2 gives details. 


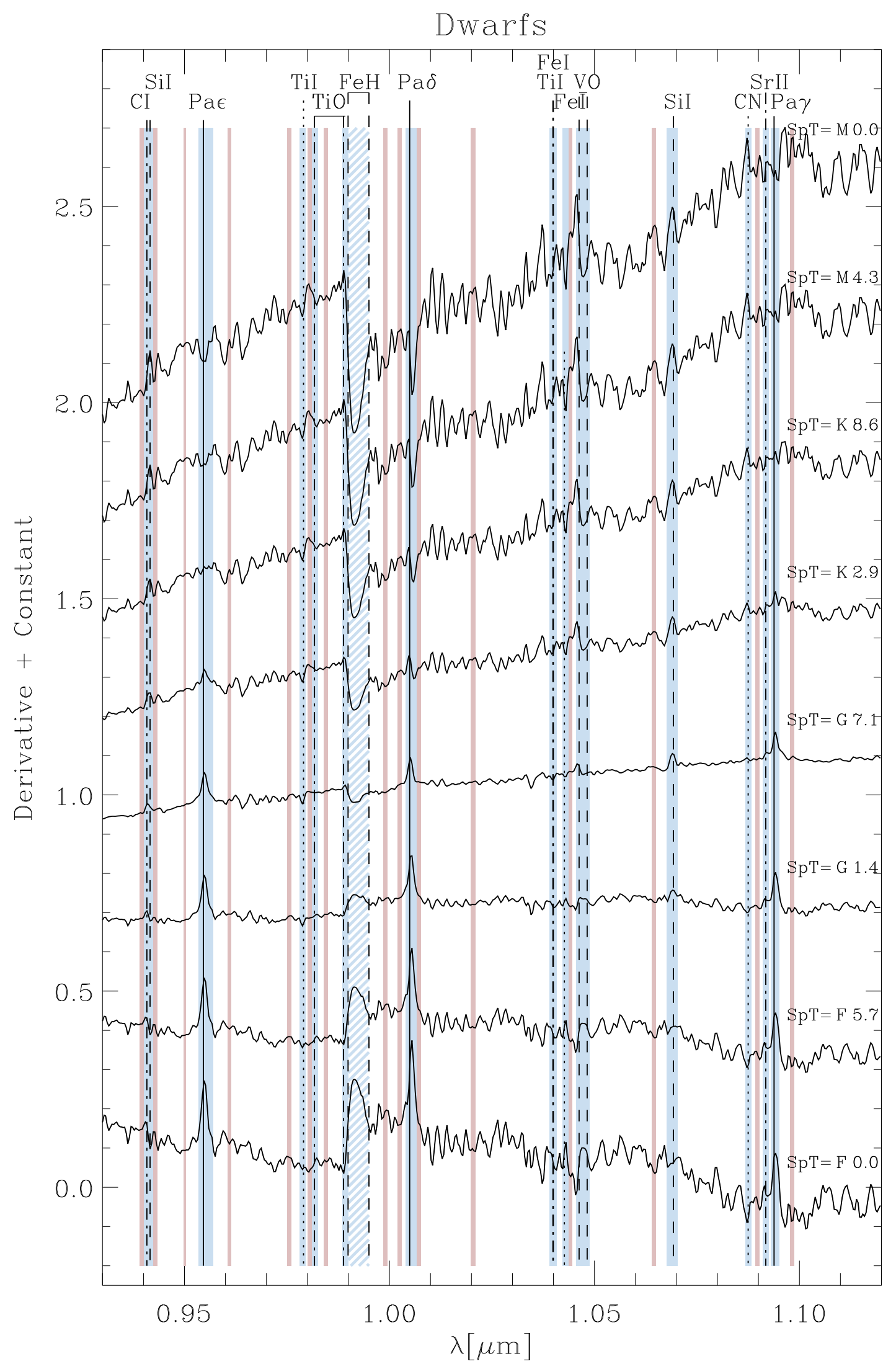

Fig. B.2. Y atmospheric window sensitivity map for SpT of dwarfs. Figure 2 gives details. 
L. Morelli et al.: The Infrared Telescope Facility (IRTF) spectral library. II.

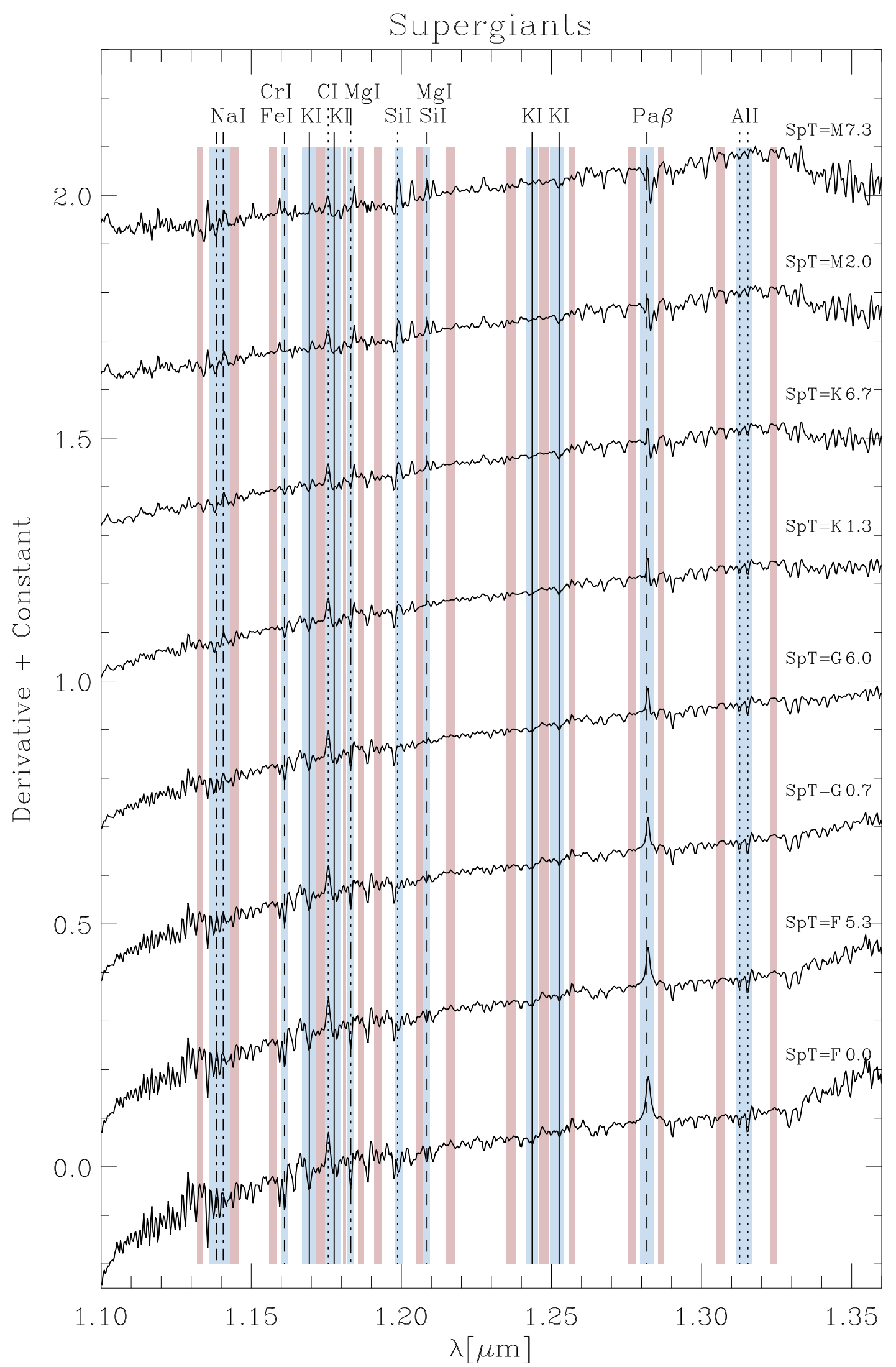

Fig. B.3. J atmospheric window sensitivity map for $\mathrm{SpT}$ of supergiants. Figure 2 gives details. 


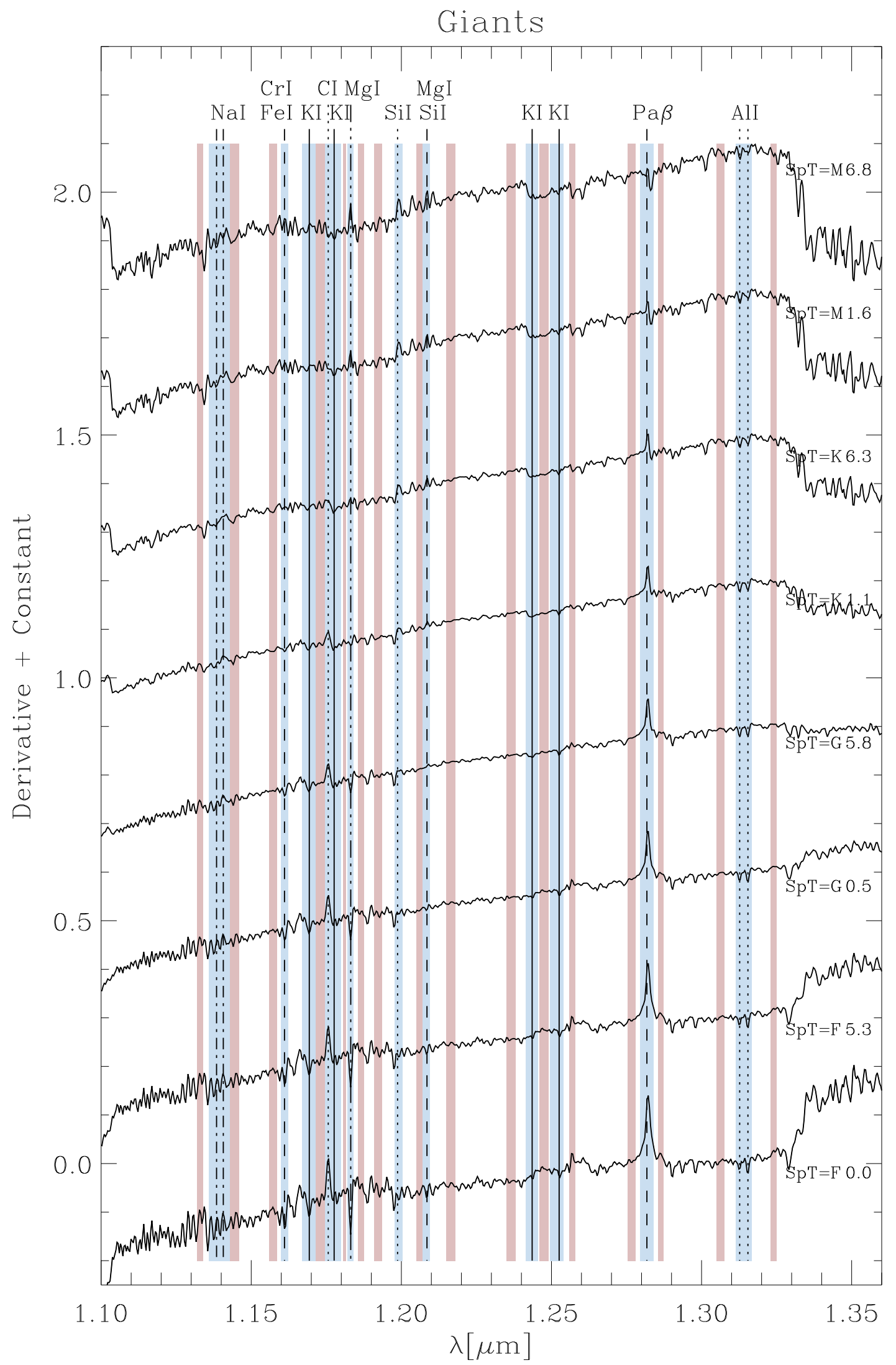

Fig. B.4. J atmospheric window sensitivity map for $\mathrm{SpT}$ of giants. Figure B.3 gives details. 
L. Morelli et al.: The Infrared Telescope Facility (IRTF) spectral library. II.

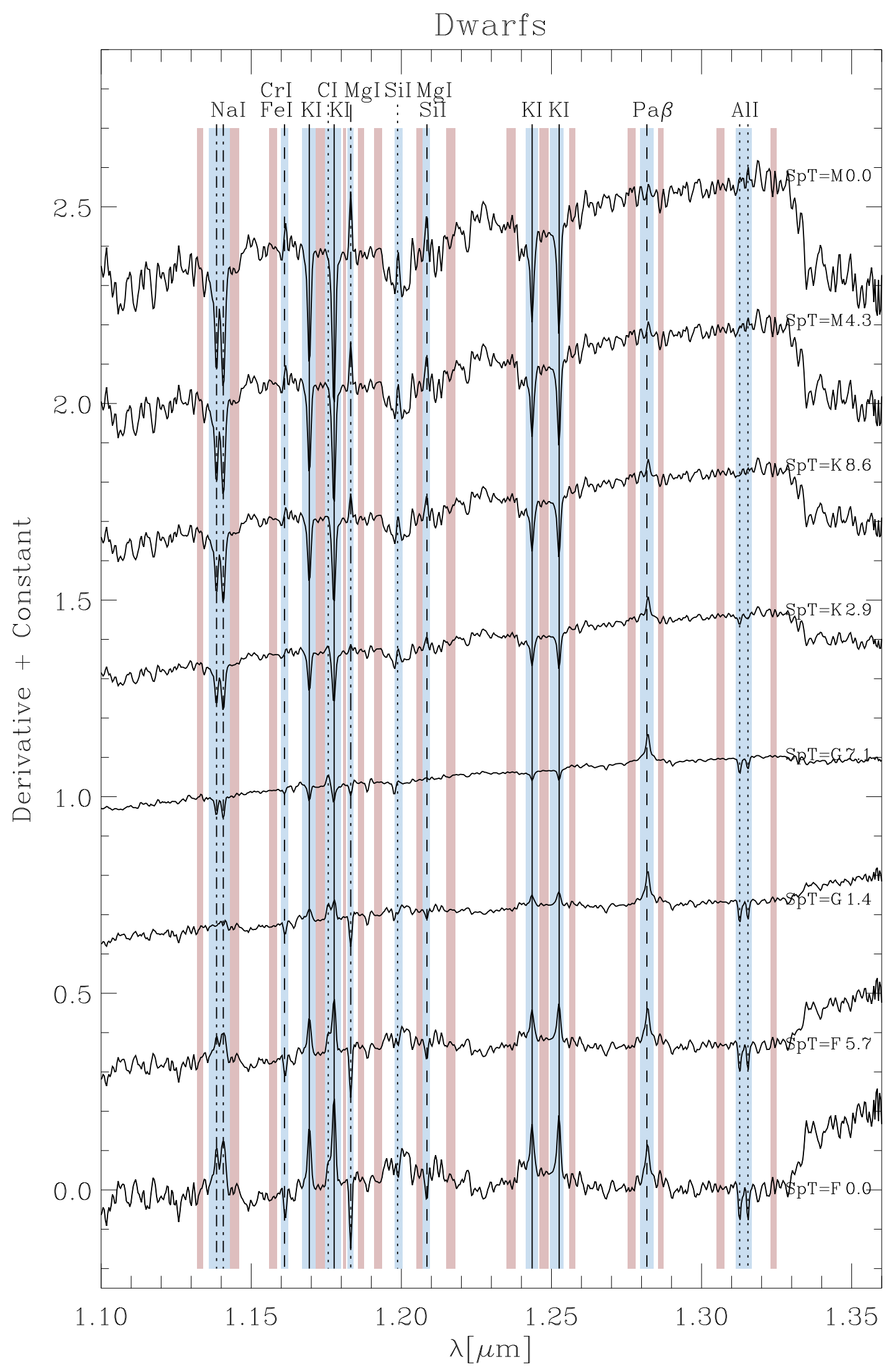

Fig. B.5. J atmospheric window sensitivity map for SpT of dwarfs. See Fig. B.3 for details. 


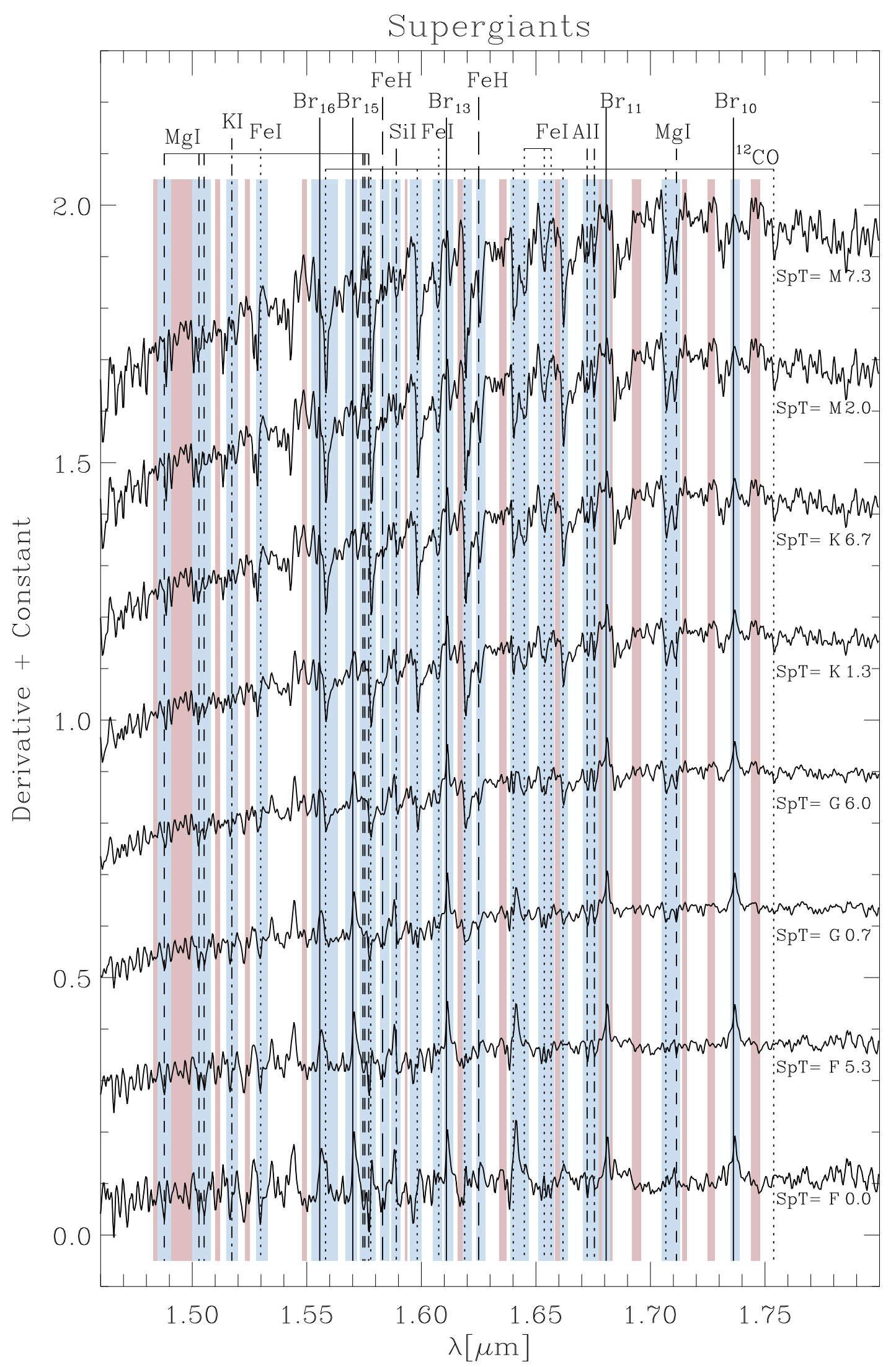

Fig. B.6. $\mathrm{H}$ atmospheric window sensitivity map for $\mathrm{SpT}$ of supergiants. Figure 2 gives details. 
L. Morelli et al.: The Infrared Telescope Facility (IRTF) spectral library. II.

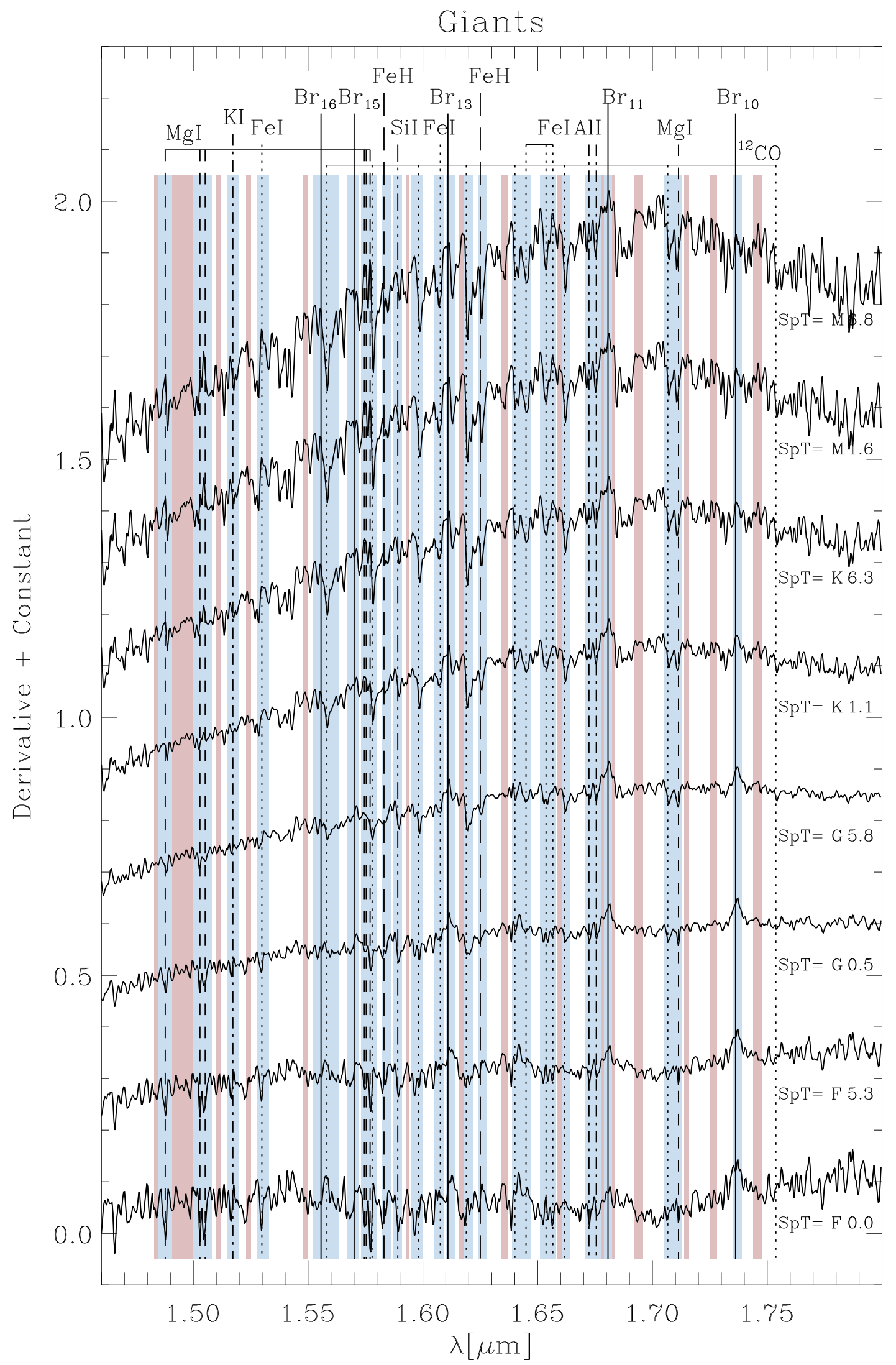

Fig. B.7. $\mathrm{H}$ atmospheric window sensitivity map for $\mathrm{SpT}$ of giants. Figure B.6 gives details. 


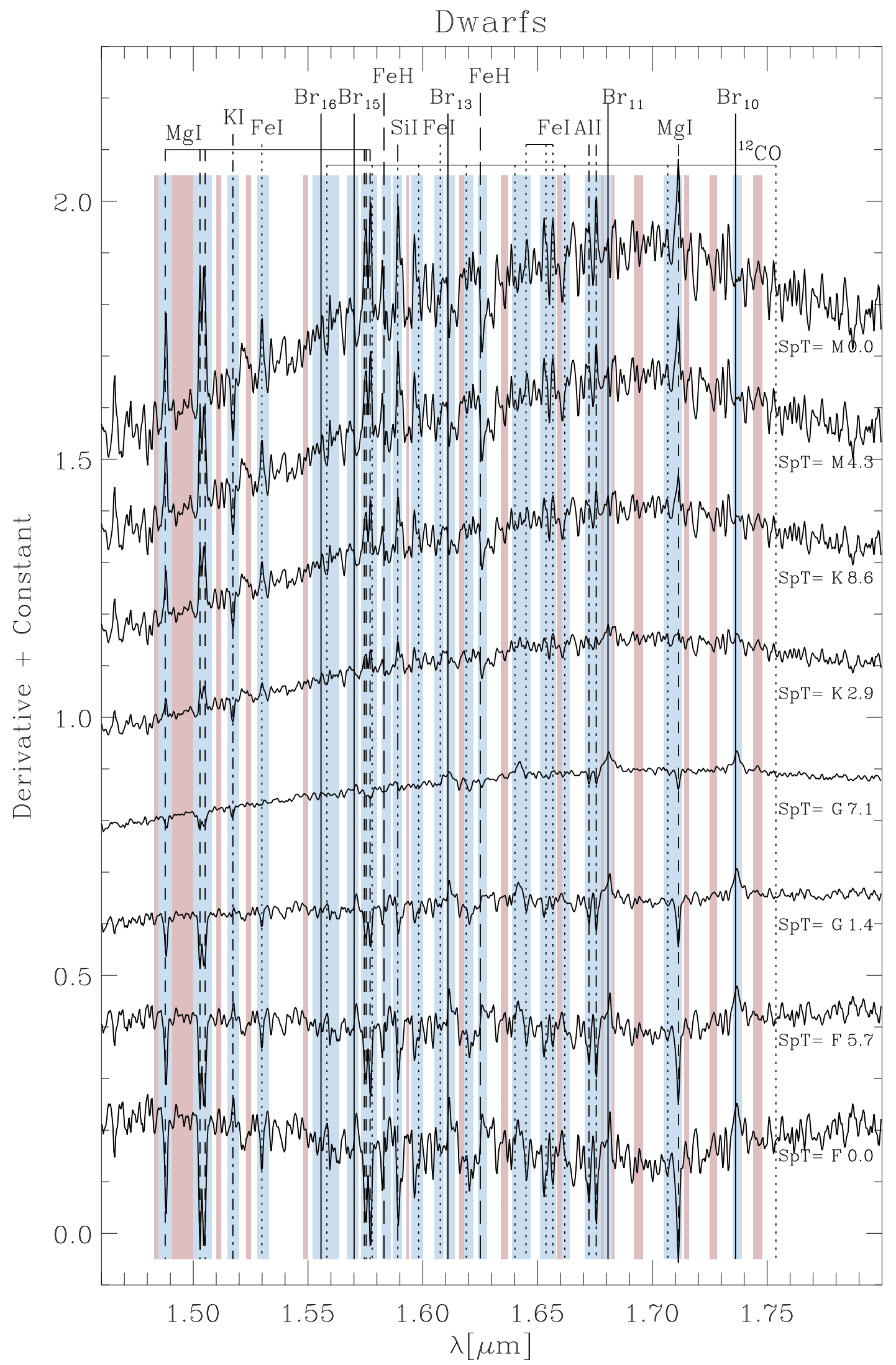

Fig. B.8. $\mathrm{H}$ atmospheric window sensitivity map for $\mathrm{SpT}$ of dwarfs. Figure B.6 gives details. 
L. Morelli et al.: The Infrared Telescope Facility (IRTF) spectral library. II.

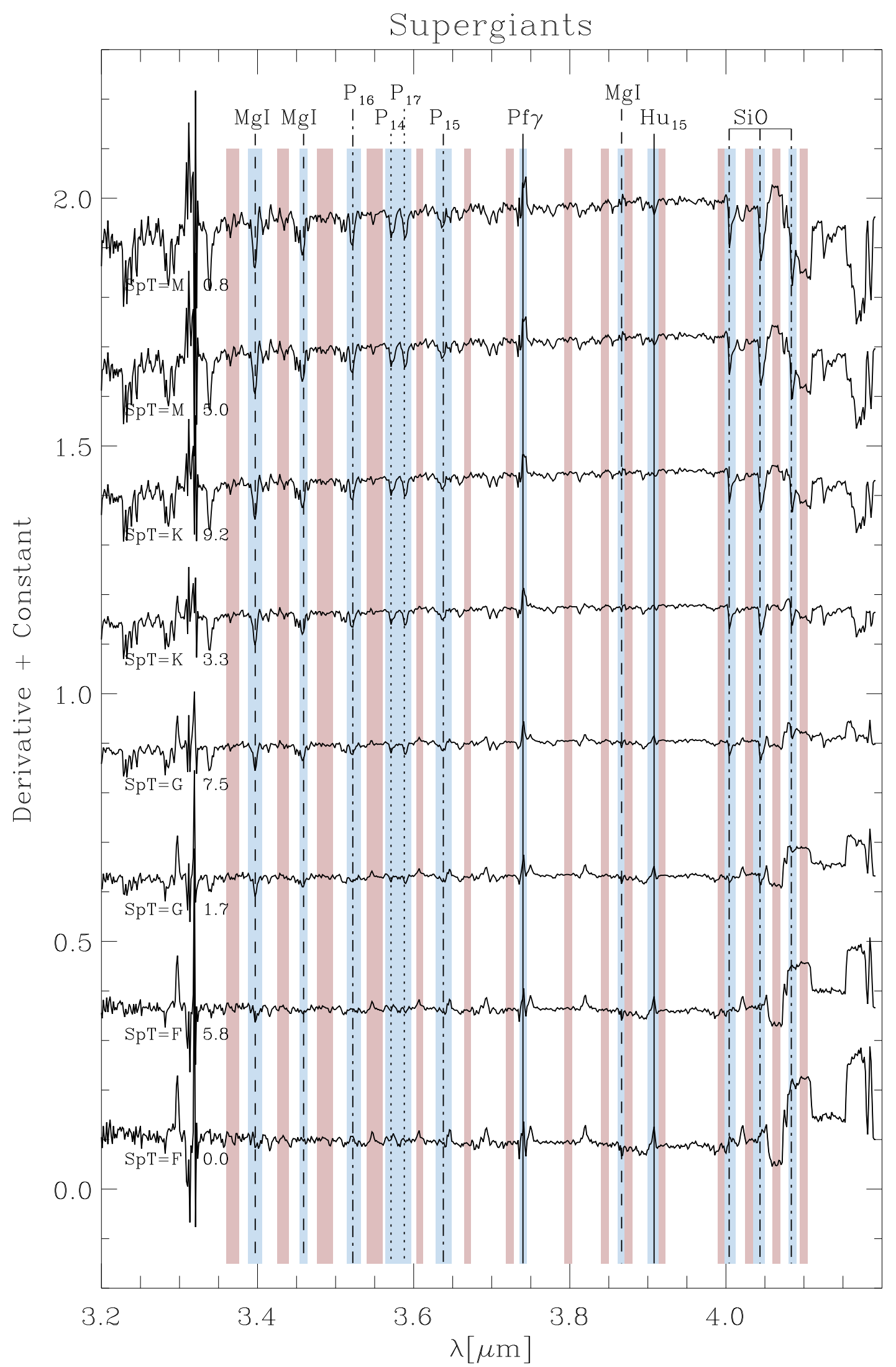

Fig. B.9. $\mathrm{L}$ atmospheric window sensitivity map for SpT of supergiants. See Fig. 2 for details. 


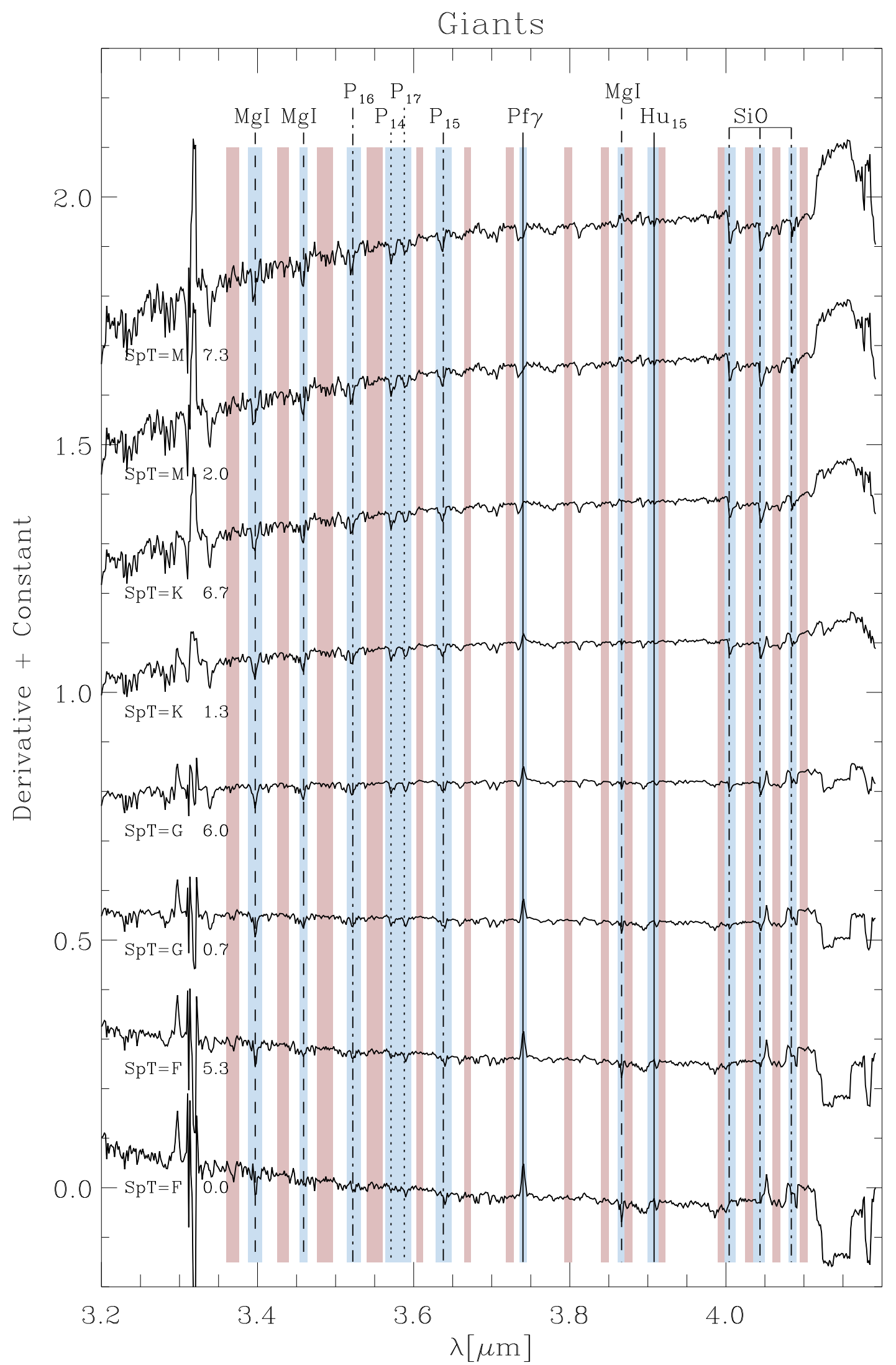

Fig. B.10. $\mathrm{L}$ atmospheric window sensitivity map for $\mathrm{SpT}$ of giants. Figure B.9 gives details. 
L. Morelli et al.: The Infrared Telescope Facility (IRTF) spectral library. II.

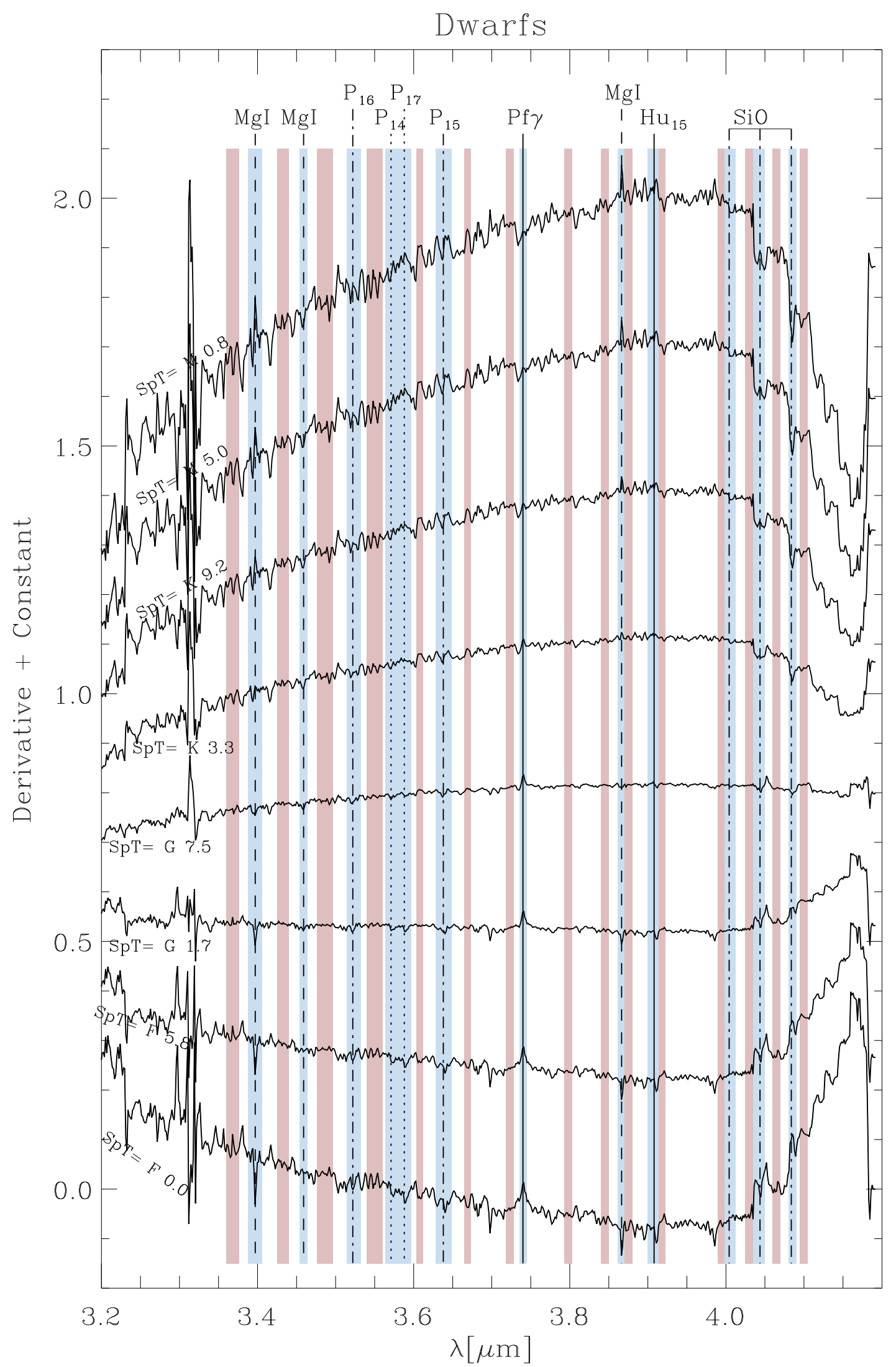

Fig. B.11. L atmospheric window sensitivity map for $\mathrm{SpT}$ of dwarfs. Figure B.9 gives details. 


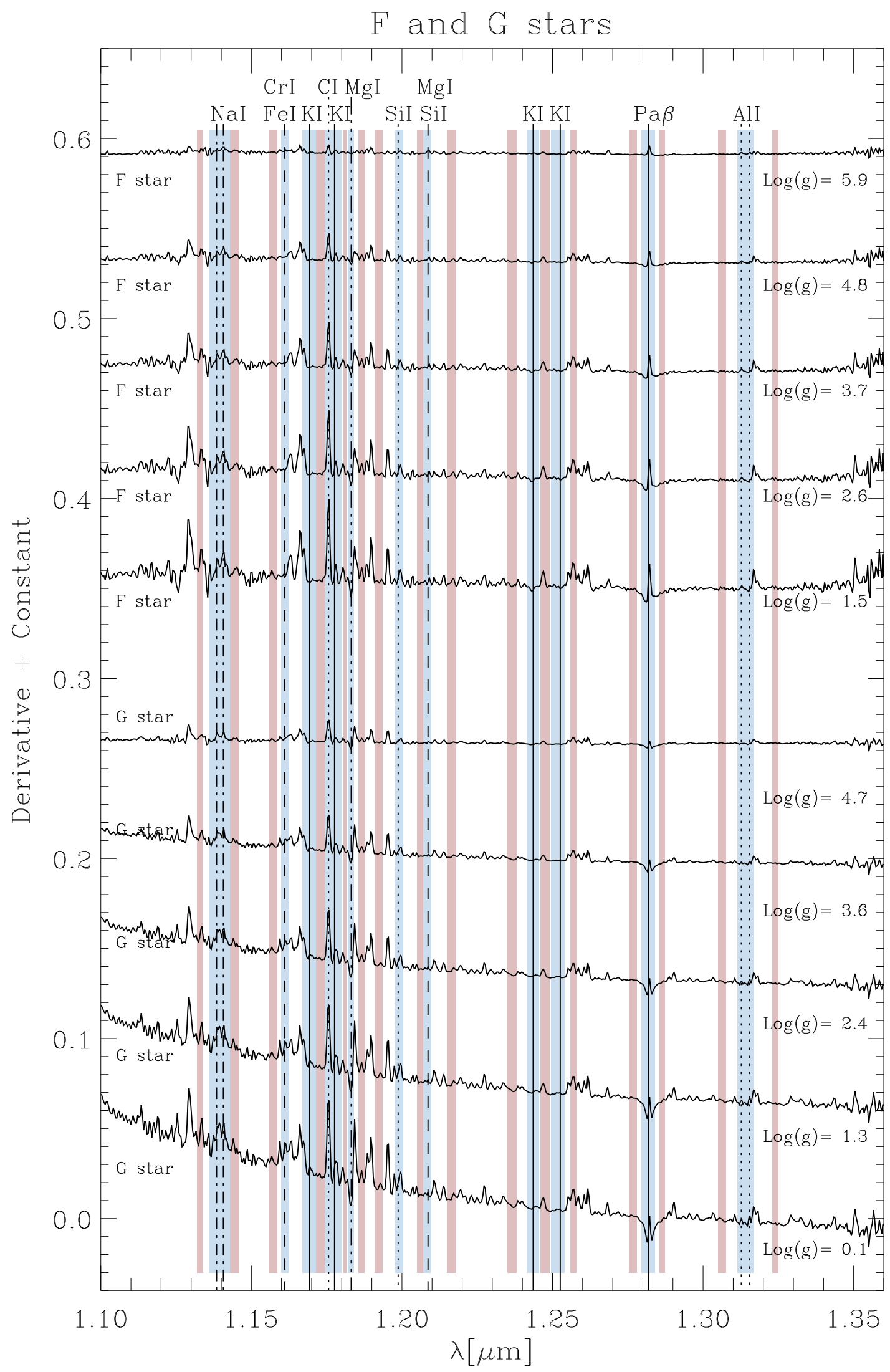

Fig. B.12. J atmospheric window sensitivity map for surface gravity of F- (top) and G-type stars (bottom). Figure 3 gives details. 
L. Morelli et al.: The Infrared Telescope Facility (IRTF) spectral library. II.

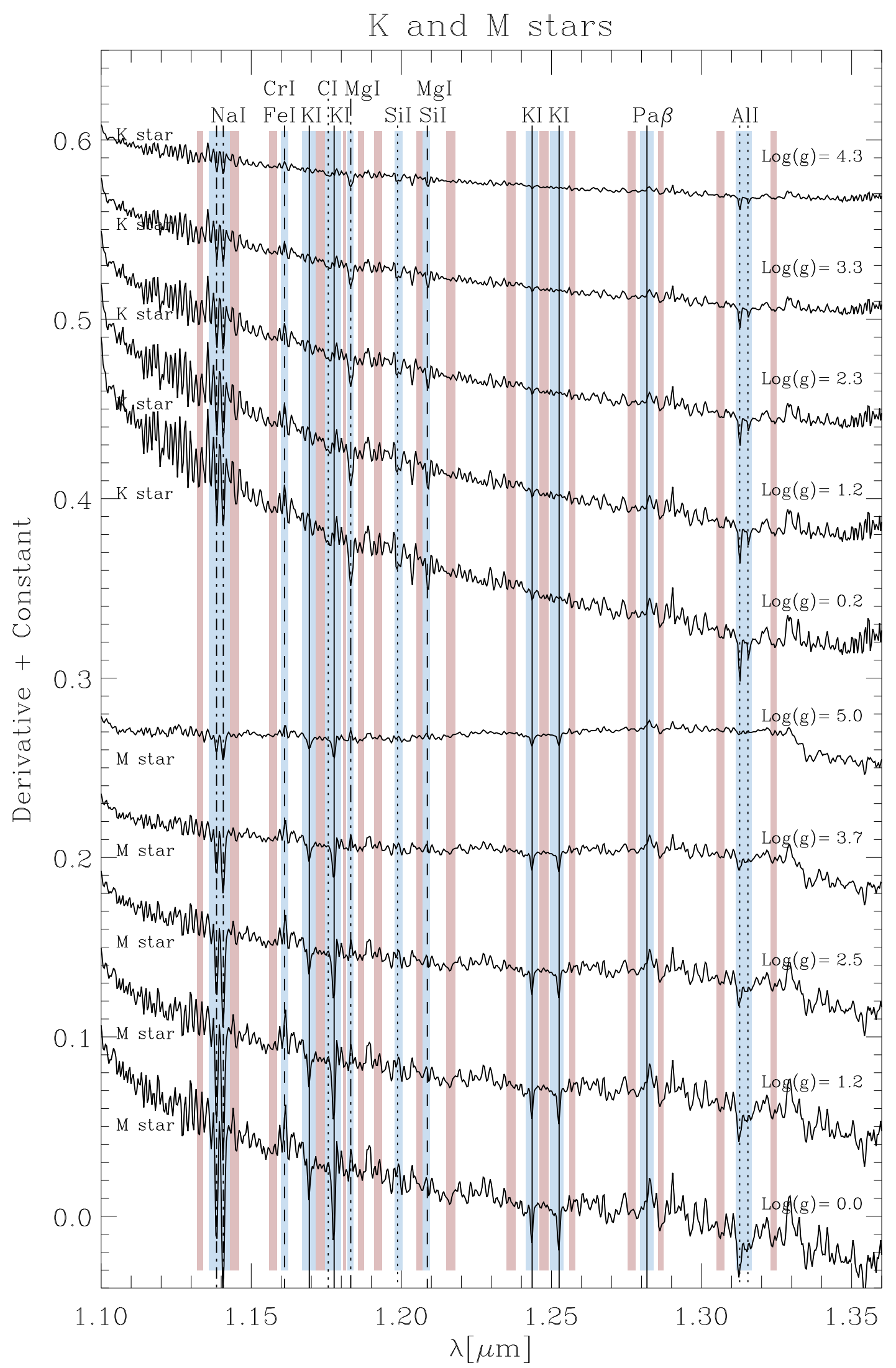

Fig. B.13. J atmospheric window sensitivity map for surface gravity of K- (top) and M-type stars (bottom). Figure 4 gives details. 


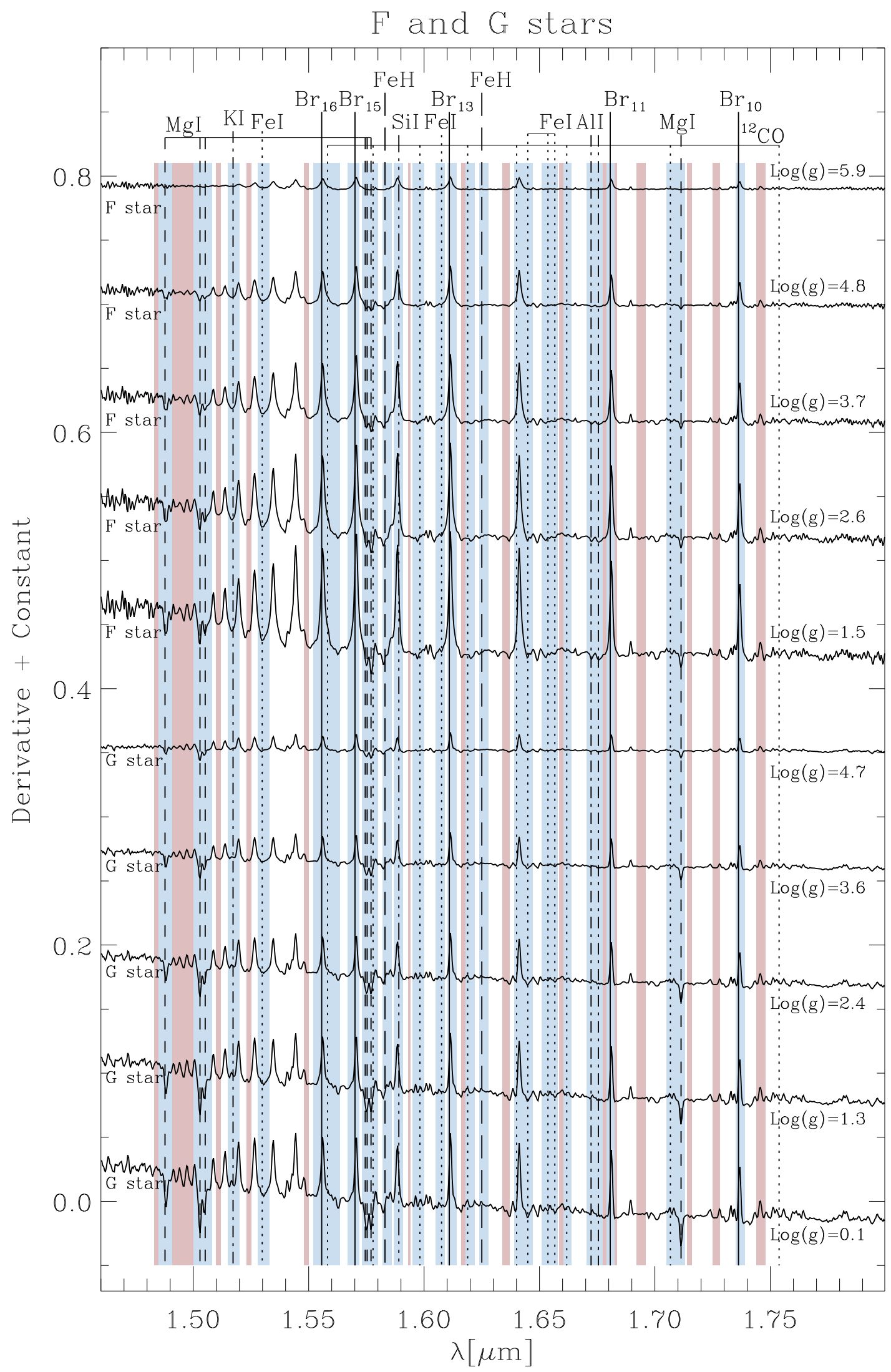

Fig. B.14. $\mathrm{H}$ atmospheric window sensitivity map for surface gravity of F- (top) and G-type stars (bottom). Figure 3 gives details. 
L. Morelli et al.: The Infrared Telescope Facility (IRTF) spectral library. II.

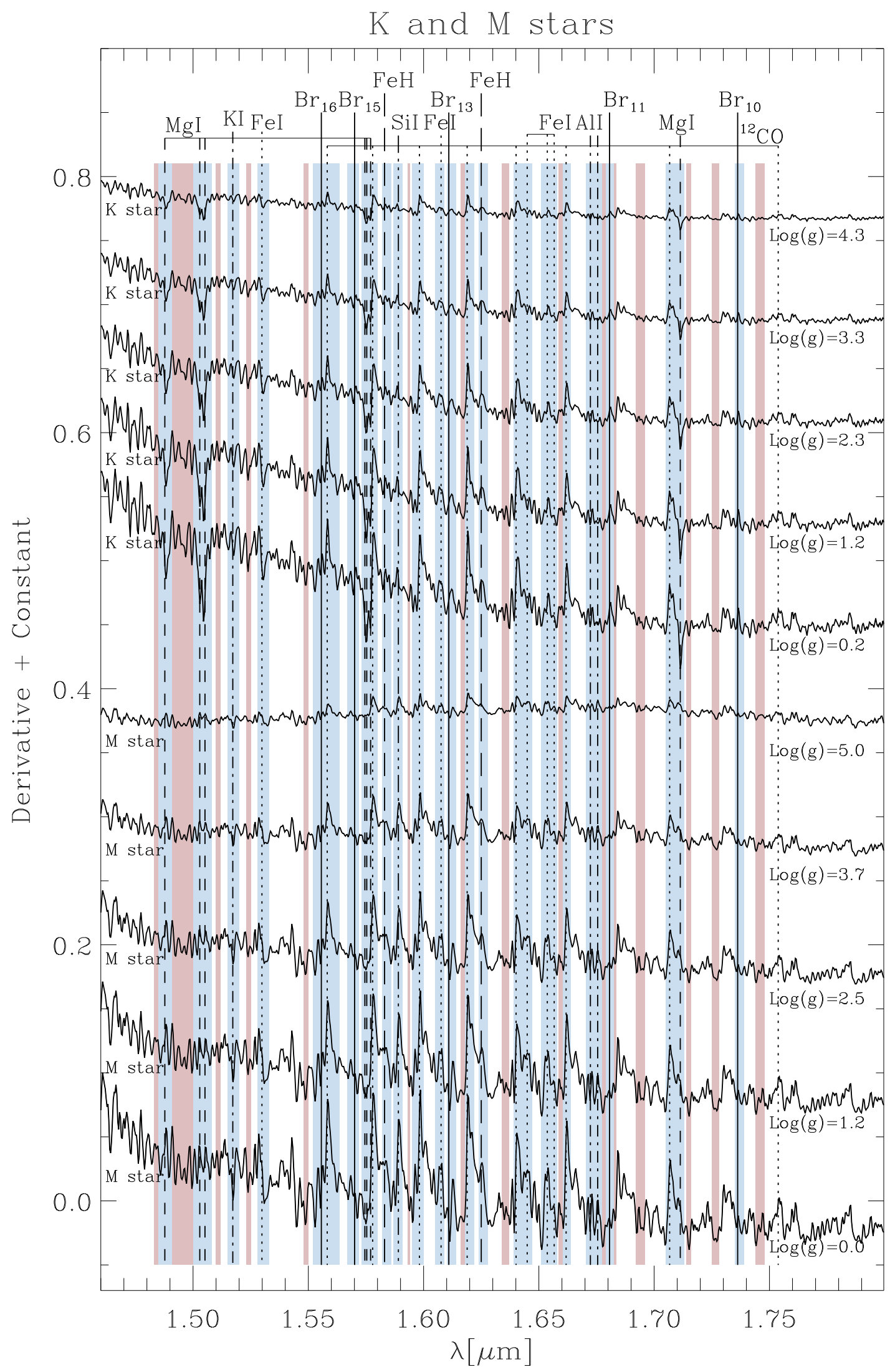

Fig. B.15. H atmospheric window sensitivity map for surface gravity of K- (top) and M-type stars (bottom). Figure 4 gives details. 


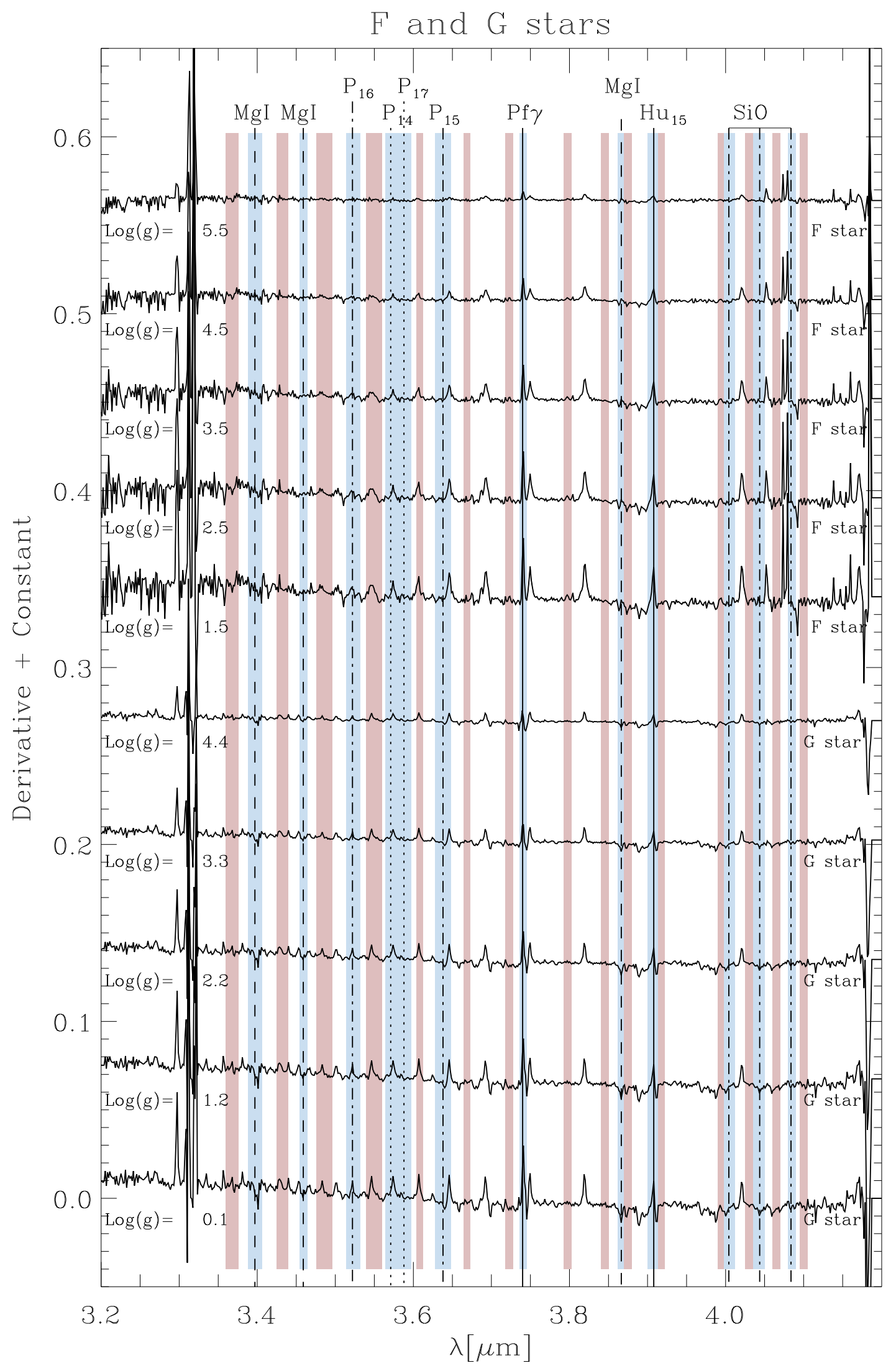

Fig. B.16. L atmospheric window sensitivity map for surface gravity of F- (top) and G-type stars (bottom). See Fig. 3 for details. 
L. Morelli et al.: The Infrared Telescope Facility (IRTF) spectral library. II.

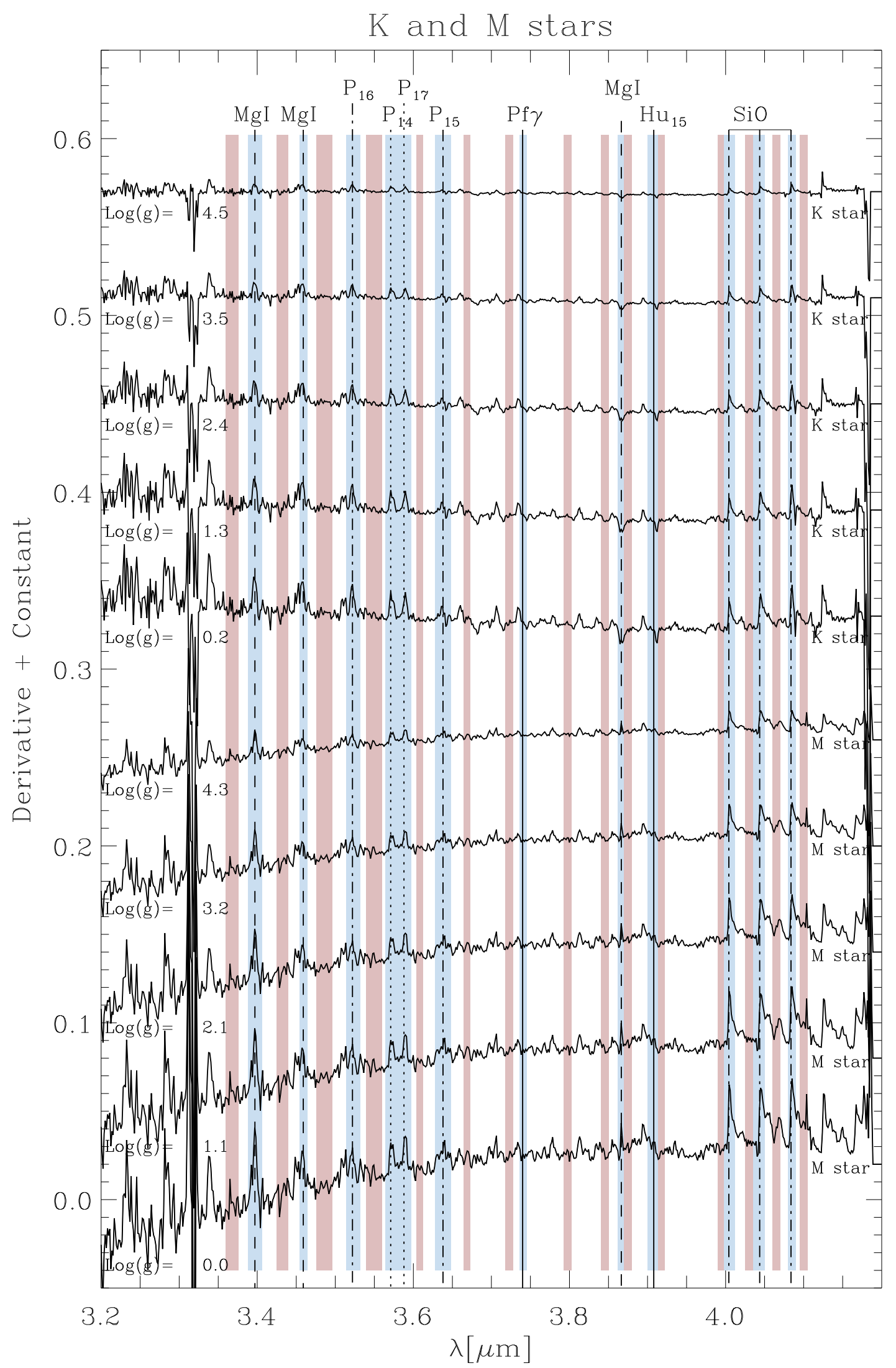

Fig. B.17. L atmospheric window sensitivity map for surface gravity of K- (top) and M-type stars (bottom). Figure 4 gives details. 


\section{Appendix C: Broadening effects}

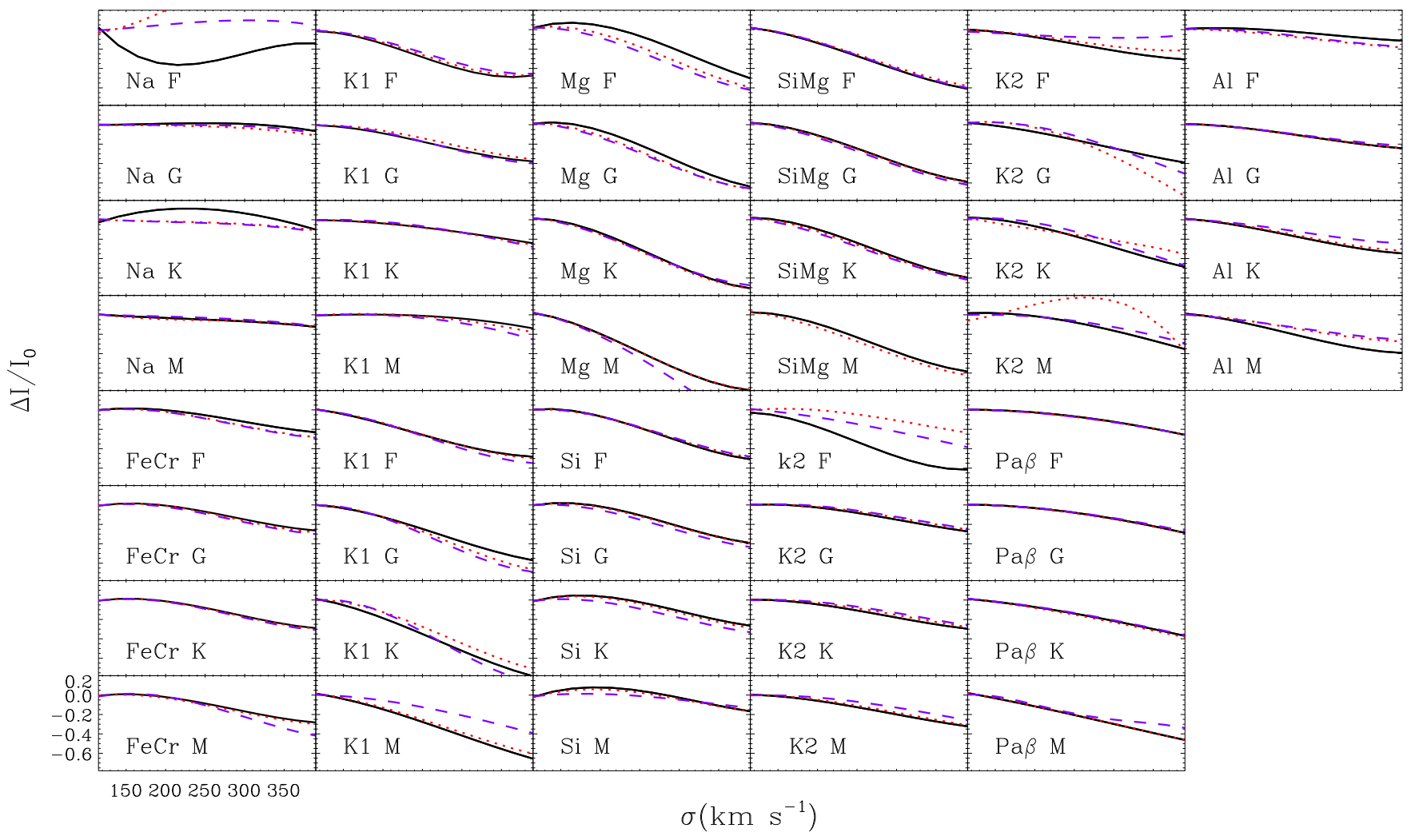

Fig. C.1. Sensitivity of the indices in the $\mathrm{J}$ atmospheric window to the velocity dispersion broadening. Figure 8 gives details. 
L. Morelli et al.: The Infrared Telescope Facility (IRTF) spectral library. II.
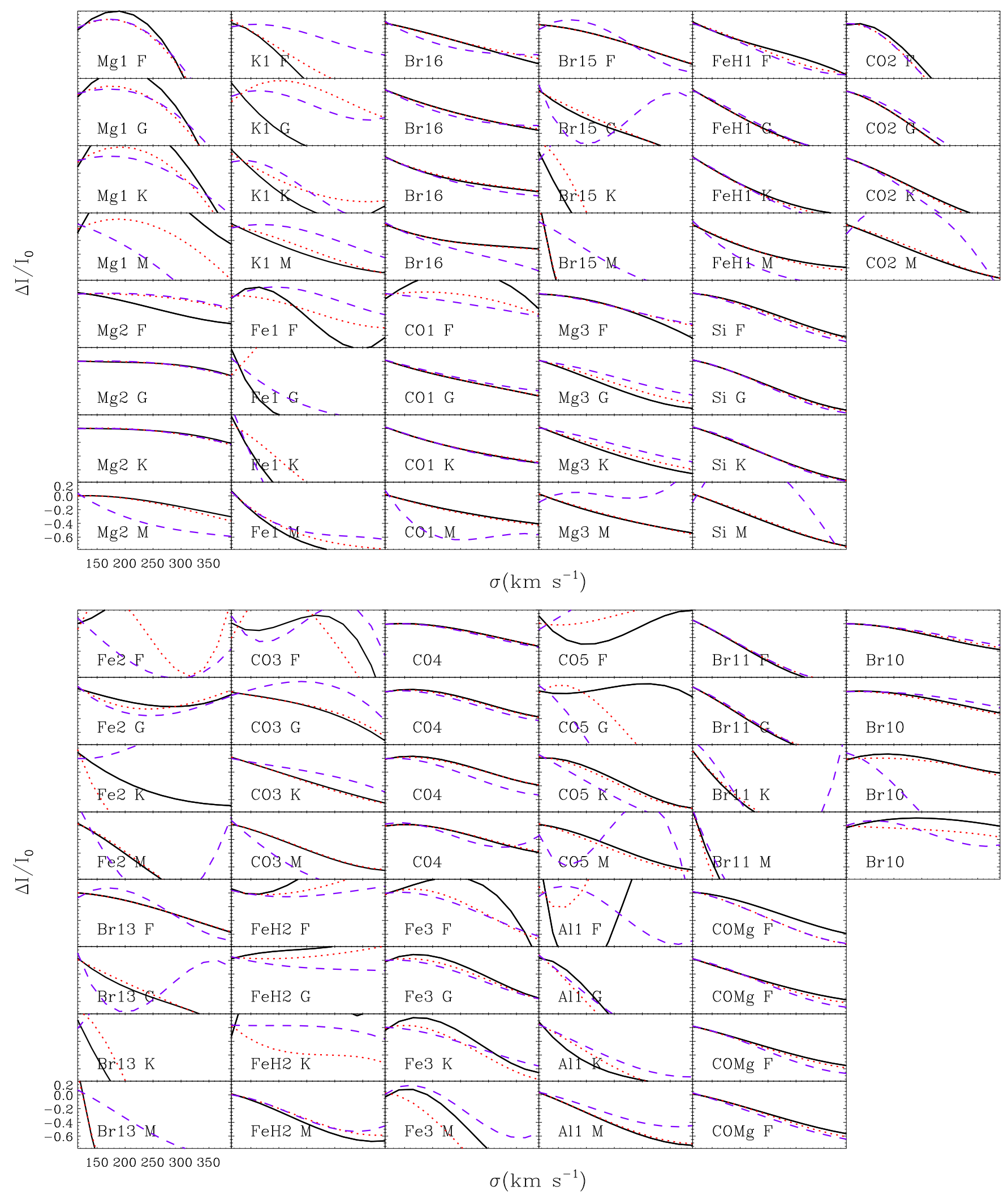

Fig. C.2. Sensitivity of the indices in the $\mathrm{H}$ atmospheric window to the velocity dispersion broadening. Figure 8 gives details. 


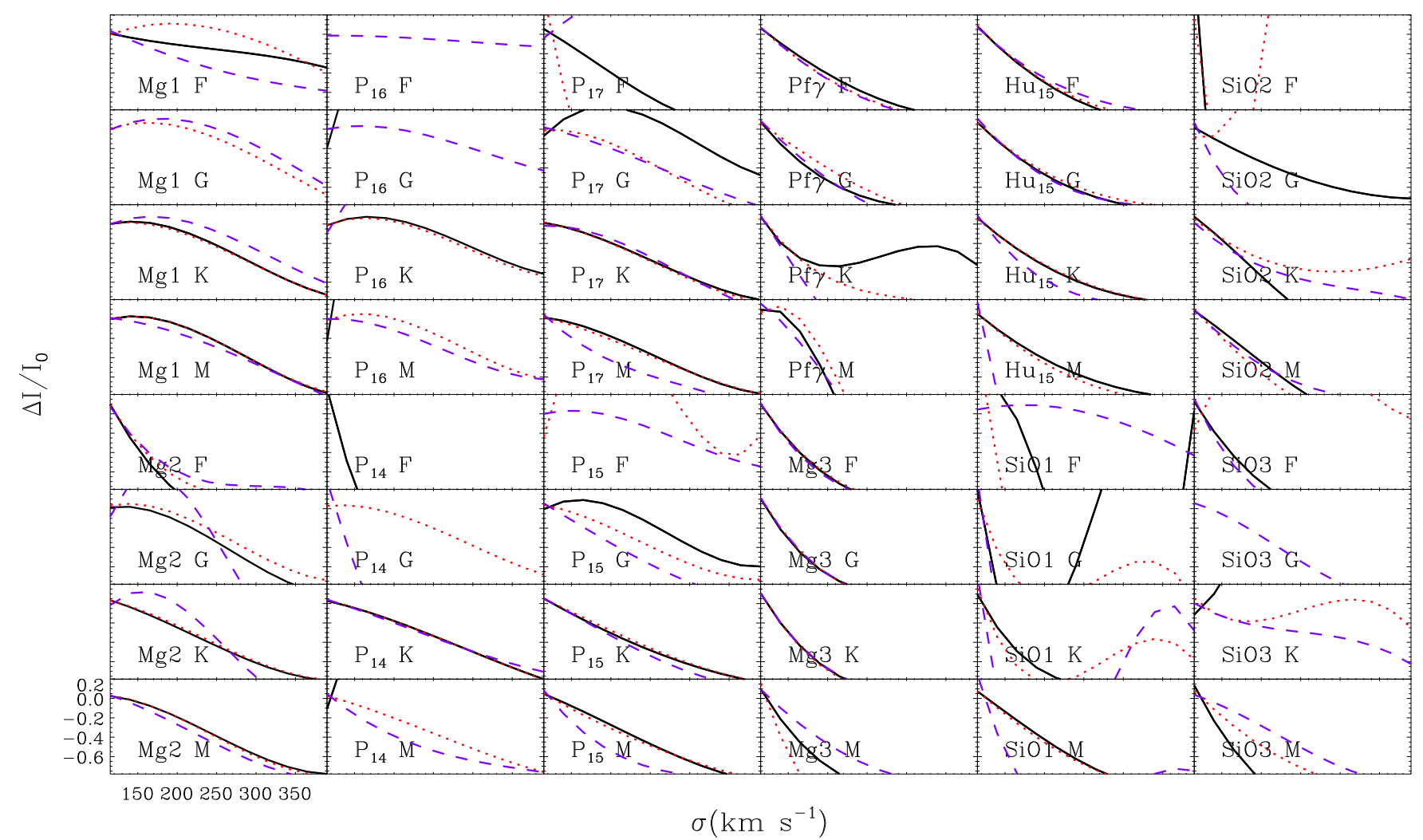

Fig. C.3. Sensitivity of the indices in the $\mathrm{L}$ atmospheric window to the velocity dispersion broadening. Figure 8 gives details.

\section{Appendix D: Behavior of the spectral indices}

The behavior of index measurements in the $\mathrm{Y}, \mathrm{J}, \mathrm{H}$, and $\mathrm{L}$ atmospheric window as functions of effective temperature, surface gravity and metallicity for stars of different luminosity classes are shown in Figs. 5, 6, and D.1-D.7.
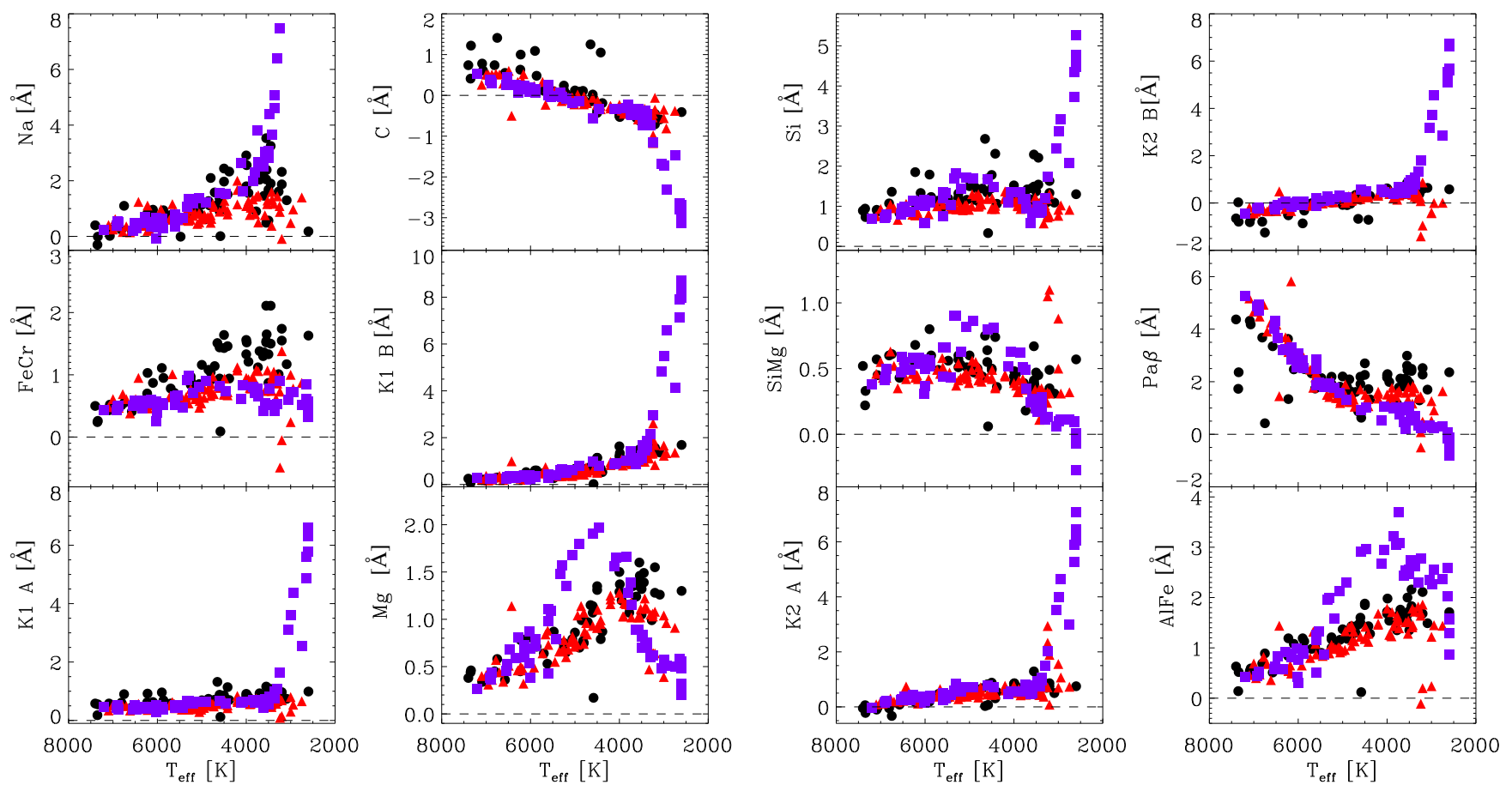

Fig. D.1. Index measurements in the J atmospheric window as functions of effective temperature. For details see Fig. 5. 
L. Morelli et al.: The Infrared Telescope Facility (IRTF) spectral library. II.
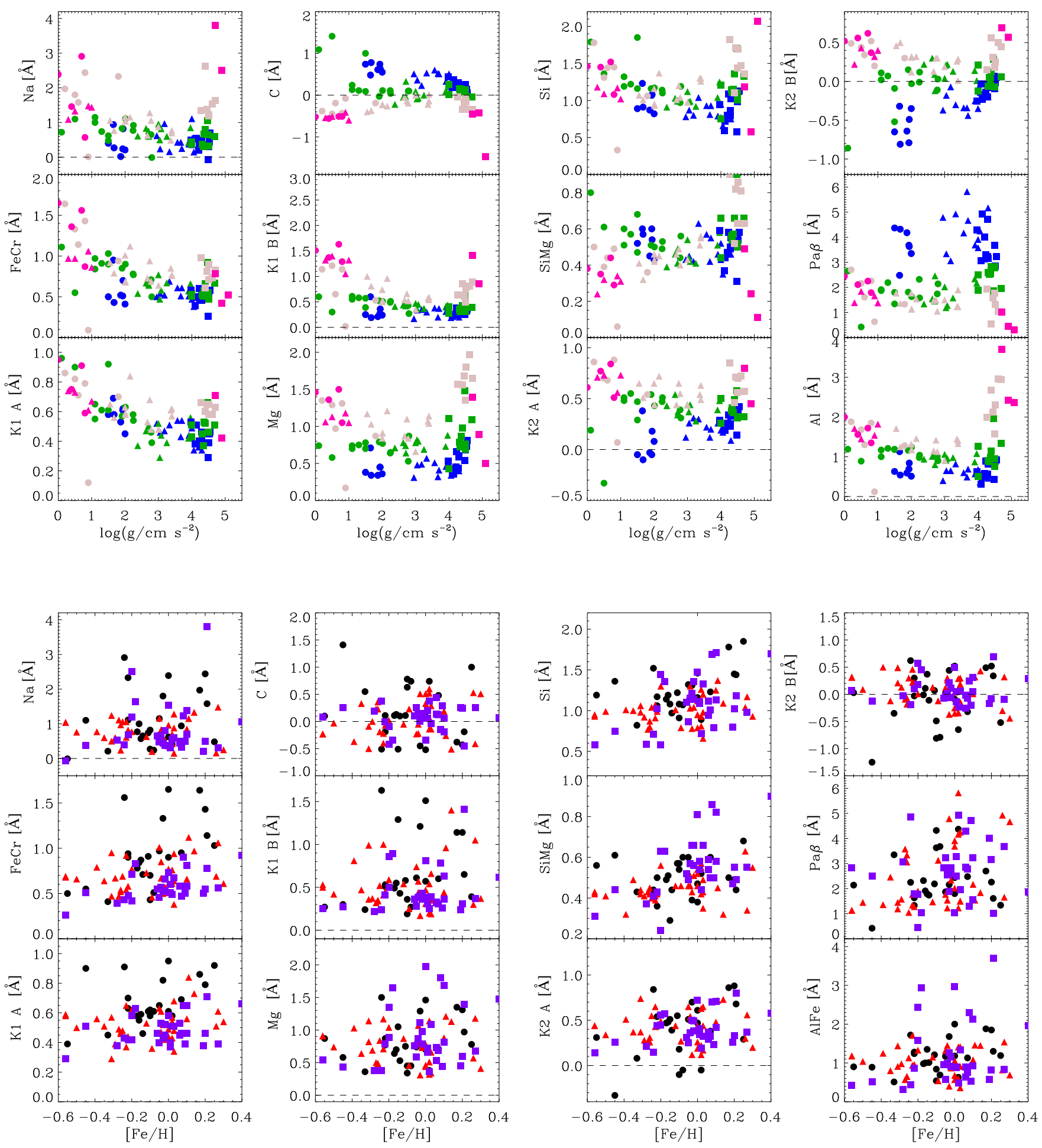

Fig. D.2. Index measurements in the $\mathrm{J}$ atmospheric window as functions of surface gravity (top panels) and metallicity (bottom panels). For details see Fig. 6. 

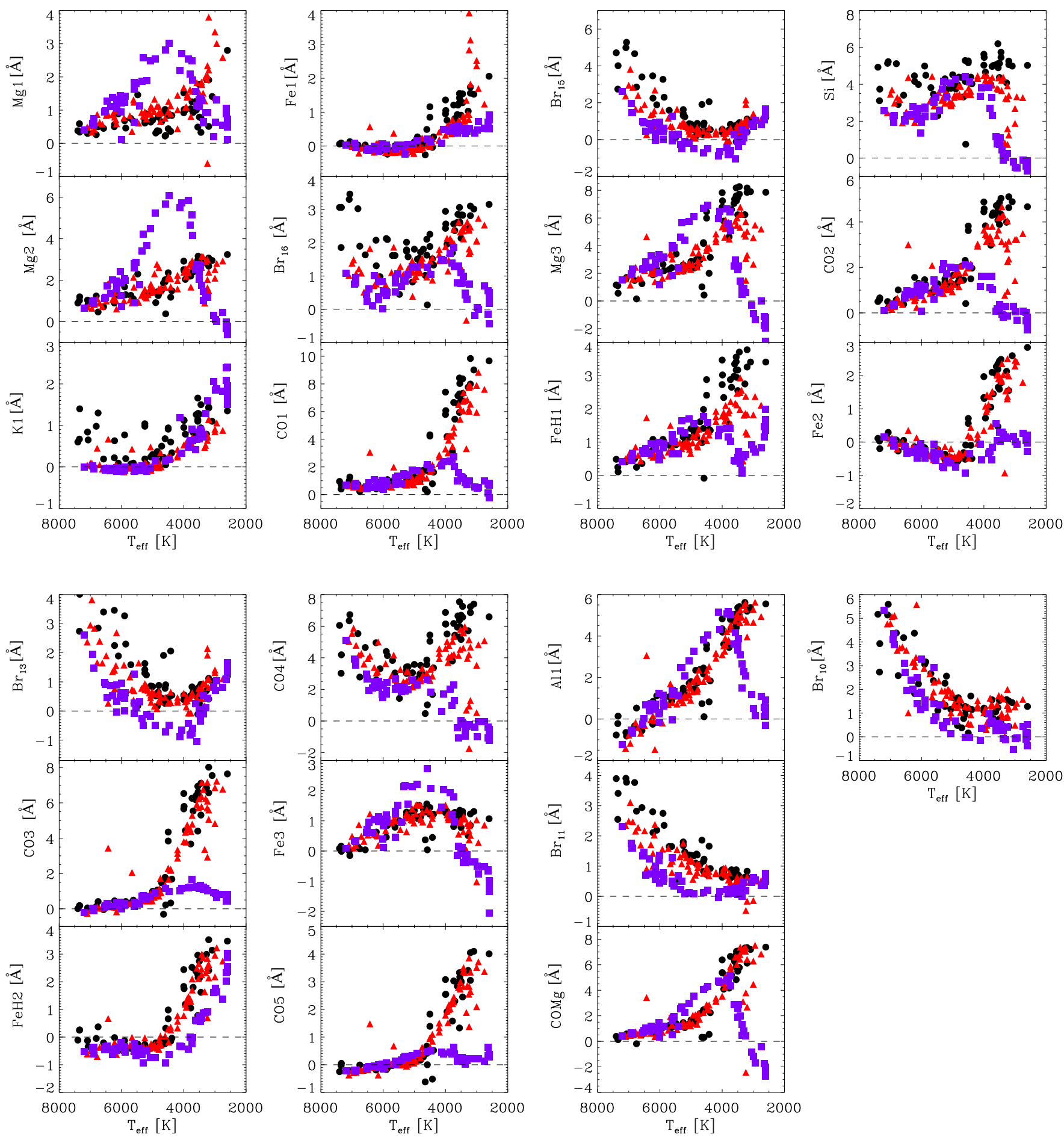

Fig. D.3. Index measurements in the $\mathrm{H}$ atmospheric window as functions of effective temperature. For details see Fig. 5. 
L. Morelli et al.: The Infrared Telescope Facility (IRTF) spectral library. II.
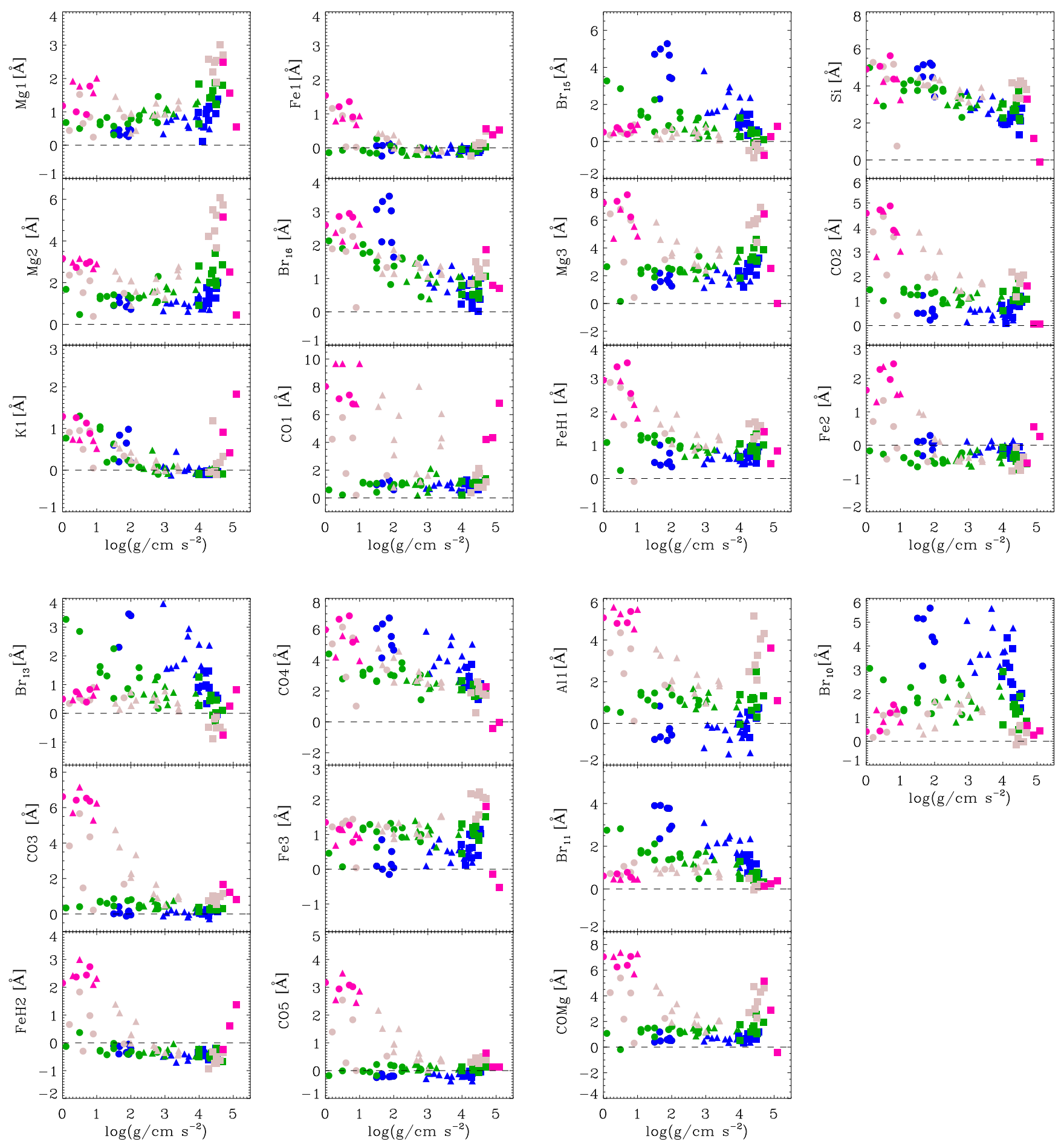

Fig. D.4. Index measurements in the $\mathrm{H}$ atmospheric window as functions of surface gravity. For details see Fig. 6. 

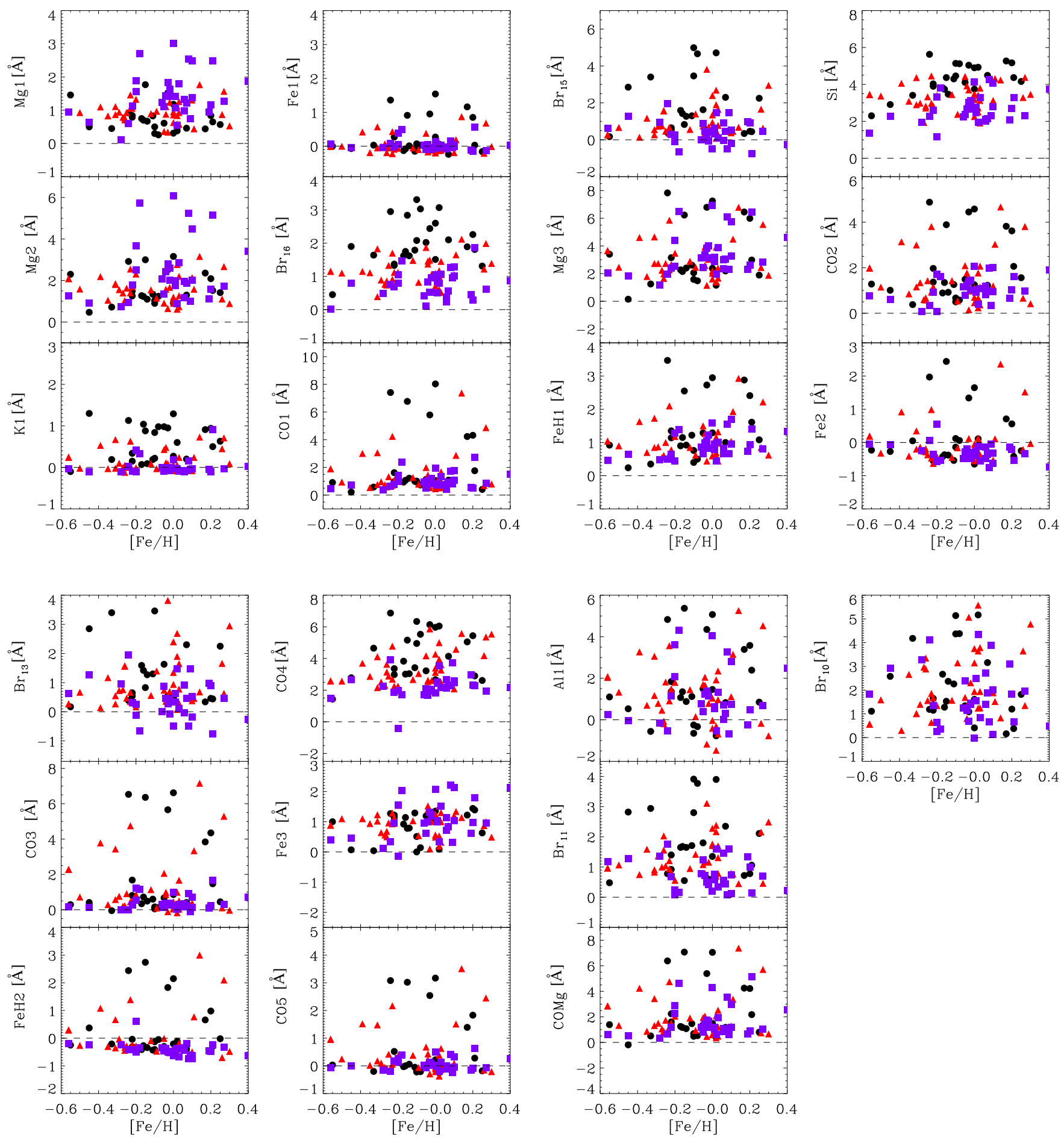

Fig. D.5. Index measurements in the $\mathrm{H}$ atmospheric window as functions of metallicity. For details see Fig. 6 . 
L. Morelli et al.: The Infrared Telescope Facility (IRTF) spectral library. II.
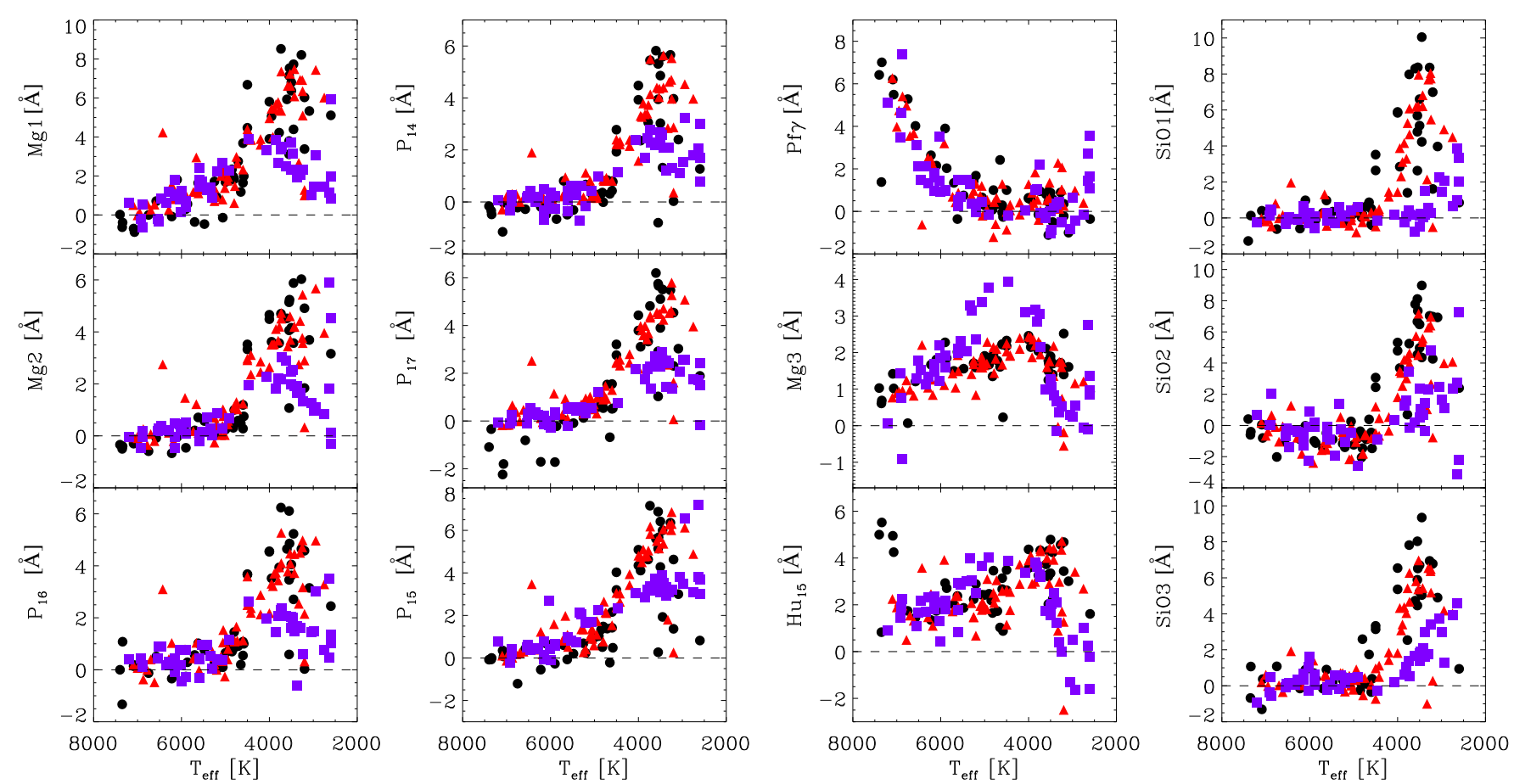

Fig. D.6. Index measurements in the $\mathrm{L}$ atmospheric window as functions of effective temperature. For details see Fig. 5. 

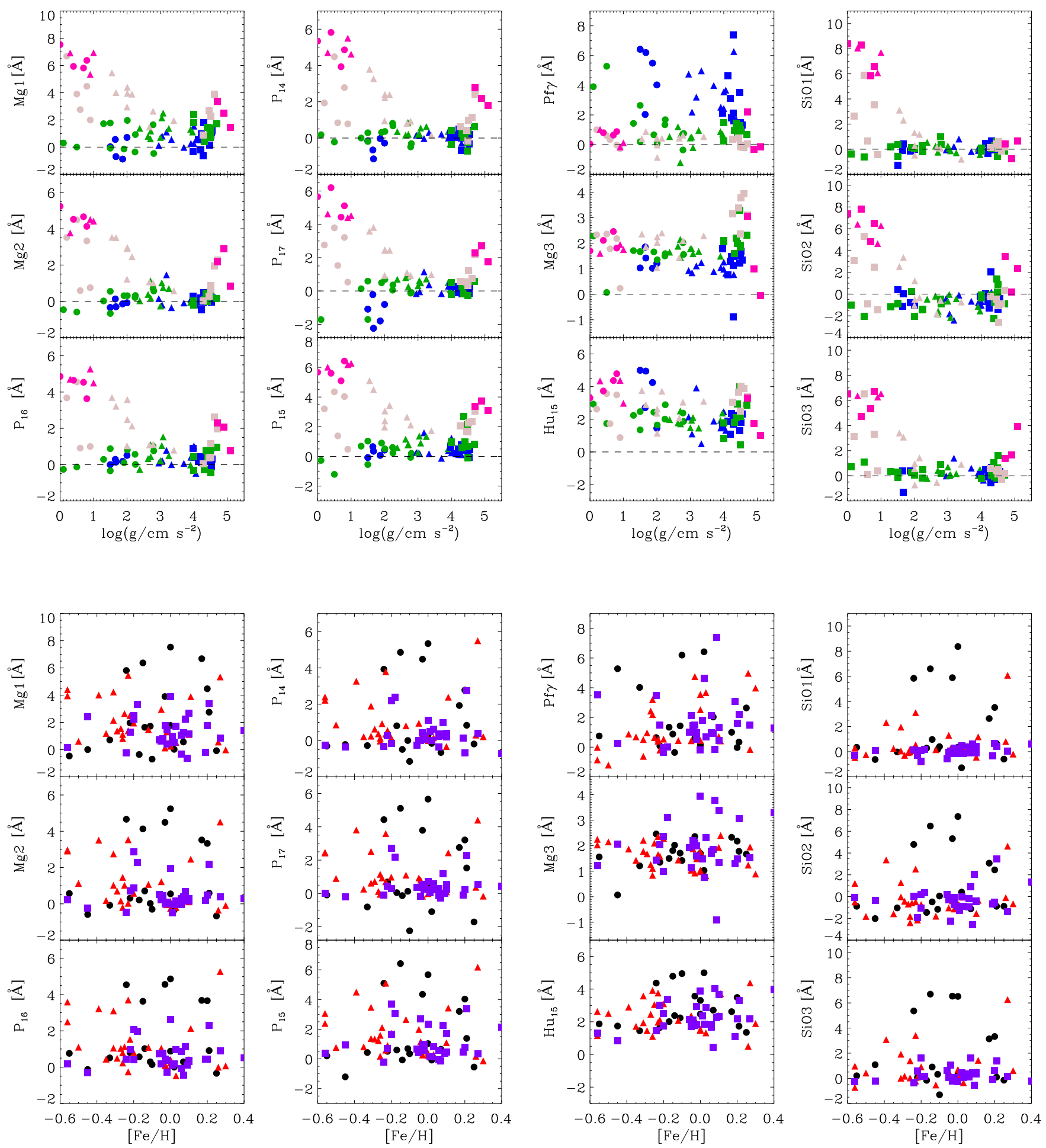

Fig. D.7. Index measurements in the $\mathrm{L}$ atmospheric window as functions of surface gravity (top panels) and metallicity (bottom panels). For details see Fig. 5. 
L. Morelli et al.: The Infrared Telescope Facility (IRTF) spectral library. II.

\section{Appendix E: Equivalent width tables}

In this section we report values measured for all the indices in the different bands. The tables are just an example and their integral version are available at the CDS.

Table E.1. Equivalent widths of the $Y$-band indices. Part A.

\begin{tabular}{|c|c|c|c|c|c|c|c|c|c|}
\hline Star IDs & $\mathrm{CSi}$ & $\mathrm{Pa} \epsilon$ & $\mathrm{Ti}$ & $\mathrm{TiO} \mathrm{A}$ & $\mathrm{TiO} \mathrm{B}$ & $\mathrm{FeH}$ & $\operatorname{Pa} \delta$ & $\mathrm{FeTi}$ & $\mathrm{Fe}$ \\
\hline \multicolumn{10}{|c|}{ Supergiants } \\
\hline HD007927 & $0.74 \pm 0.06$ & $3.07 \pm 0.65$ & $0.03 \pm 0.01$ & $0.03 \pm 0.01$ & $0.56 \pm 0.01$ & $0.49 \pm 0.02$ & $2.92 \pm 0.03$ & $-0.05 \pm 0.03$ & $-0.05 \pm 0.03$ \\
\hline HD006130 & $0.82 \pm 0.05$ & $4.11 \pm 0.46$ & $-0.05 \pm 0.03$ & $-0.02 \pm 0.02$ & $0.41 \pm 0.01$ & $0.22 \pm 0.03$ & $3.44 \pm 0.08$ & $-0.03 \pm 0.07$ & $-0.06 \pm 0.07$ \\
\hline HD135153 & $0.61 \pm 0.13$ & $3.92 \pm 0.46$ & $-0.05 \pm 0.03$ & $-0.05 \pm 0.03$ & $0.32 \pm 0.02$ & $0.07 \pm 0.05$ & $3.49 \pm 0.07$ & $-0.02 \pm 0.05$ & $-0.05 \pm 0.05$ \\
\hline HD173638 & $0.78 \pm 0.07$ & $4.34 \pm 0.42$ & $-0.04 \pm 0.01$ & $-0.02 \pm 0.02$ & $0.41 \pm 0.01$ & $0.16 \pm 0.04$ & $3.58 \pm 0.10$ & $-0.07 \pm 0.05$ & $-0.10 \pm 0.05$ \\
\hline$\cdots$ & $\cdots$ & $\ldots$ & $\ldots$ & $\cdots$ & $\ldots$ & $\cdots$ & $\ldots$ & $\cdots$ & $\ldots$ \\
\hline \multicolumn{10}{|c|}{ Giants } \\
\hline HD089025 & $0.89 \pm 0.01$ & $3.89 \pm 0.25$ & $-0.03 \pm 0.02$ & $-0.03 \pm 0.02$ & $0.31 \pm 0.01$ & $0.19 \pm 0.03$ & $2.92 \pm 0.08$ & $-0.05 \pm 0.06$ & $-0.06 \pm 0.06$ \\
\hline HD027397 & $0.68 \pm 0.02$ & $4.89 \pm 0.21$ & $-0.05 \pm 0.02$ & $-0.06 \pm 0.02$ & $0.33 \pm 0.01$ & $0.09 \pm 0.03$ & $3.51 \pm 0.10$ & $-0.01 \pm 0.03$ & $-0.01 \pm 0.03$ \\
\hline HD013174 & $0.92 \pm 0.01$ & $3.67 \pm 0.23$ & $-0.06 \pm 0.01$ & $-0.06 \pm 0.02$ & $0.29 \pm 0.01$ & $0.11 \pm 0.04$ & $2.82 \pm 0.09$ & $-0.05 \pm 0.04$ & $-0.06 \pm 0.04$ \\
\hline HD40535 & $0.89 \pm 0.02$ & $3.49 \pm 0.22$ & $-0.05 \pm 0.02$ & $-0.04 \pm 0.02$ & $0.30 \pm 0.01$ & $0.05 \pm 0.02$ & $2.68 \pm 0.08$ & $-0.02 \pm 0.03$ & $-0.02 \pm 0.03$ \\
\hline$\ldots$ & $\ldots$ & $\ldots$ & $\ldots$ & $\ldots$ & $\ldots$ & $\ldots$ & $\ldots$ & $\ldots$ & $\ldots$ \\
\hline \multicolumn{10}{|c|}{ Dwarfs } \\
\hline HD108519 & $0.73 \pm 0.04$ & $4.45 \pm 0.21$ & $-0.03 \pm 0.01$ & $-0.02 \pm 0.01$ & $0.24 \pm 0.02$ & $0.11 \pm 0.05$ & $3.30 \pm 0.09$ & $-0.03 \pm 0.03$ & $-0.02 \pm 0.03$ \\
\hline HD213135 & $0.74 \pm 0.04$ & $3.43 \pm 0.20$ & $-0.02 \pm 0.01$ & $-0.05 \pm 0.01$ & $0.19 \pm 0.00$ & $0.04 \pm 0.01$ & $2.71 \pm 0.08$ & $0.01 \pm 0.03$ & $-0.02 \pm 0.03$ \\
\hline HD113139 & $0.95 \pm 0.02$ & $3.50 \pm 0.22$ & $-0.05 \pm 0.02$ & $-0.05 \pm 0.01$ & $0.25 \pm 0.01$ & $0.09 \pm 0.03$ & $2.78 \pm 0.09$ & $-0.03 \pm 0.02$ & $-0.03 \pm 0.02$ \\
\hline HD026015 & $0.89 \pm 0.03$ & $3.62 \pm 0.18$ & $-0.05 \pm 0.02$ & $-0.06 \pm 0.02$ & $0.29 \pm 0.01$ & $0.10 \pm 0.04$ & $2.74 \pm 0.09$ & $0.02 \pm 0.03$ & $-0.03 \pm 0.03$ \\
\hline$\ldots$ & $\ldots$ & $\ldots$ & $\ldots$ & $\ldots$ & $\ldots$ & $\ldots$ & $\ldots$ & $\ldots$ & $\ldots$ \\
\hline
\end{tabular}

Table E.2. Equivalent widths of the $Y$-band indices. Part B.

\begin{tabular}{|c|c|c|c|c|c|}
\hline Star IDs & VO & $\mathrm{Si}$ & $\mathrm{CN}$ & $\mathrm{Sr}$ & $\operatorname{Pa} \gamma$ \\
\hline \multicolumn{6}{|c|}{ Supergiants } \\
\hline HD007927 & $0.87 \pm 0.04$ & $3.26 \pm 0.02$ & $0.51 \pm 0.02$ & $1.00 \pm 0.01$ & $2.56 \pm 0.02$ \\
\hline HD006130 & $0.78 \pm 0.09$ & $2.01 \pm 0.02$ & $0.40 \pm 0.01$ & $0.70 \pm 0.03$ & $3.11 \pm 0.06$ \\
\hline HD135153 & $0.68 \pm 0.06$ & $1.73 \pm 0.02$ & $0.43 \pm 0.01$ & $0.71 \pm 0.03$ & $3.18 \pm 0.07$ \\
\hline HD173638 & $0.67 \pm 0.08$ & $2.08 \pm 0.03$ & $0.50 \pm 0.03$ & $0.77 \pm 0.03$ & $3.29 \pm 0.06$ \\
\hline \multicolumn{6}{|c|}{ Giants } \\
\hline HD089025 & $0.52 \pm 0.11$ & $1.50 \pm 0.03$ & $0.41 \pm 0.03$ & $0.65 \pm 0.03$ & $2.98 \pm 0.08$ \\
\hline HD027397 & $0.53 \pm 0.06$ & $1.04 \pm 0.03$ & $0.35 \pm 0.02$ & $0.74 \pm 0.01$ & $3.59 \pm 0.03$ \\
\hline HD013174 & $0.53 \pm 0.06$ & $1.82 \pm 0.04$ & $0.38 \pm 0.03$ & $0.51 \pm 0.02$ & $2.83 \pm 0.05$ \\
\hline HD40535 & $0.65 \pm 0.05$ & $1.49 \pm 0.02$ & $0.37 \pm 0.02$ & $0.51 \pm 0.01$ & $2.70 \pm 0.03$ \\
\hline \multicolumn{6}{|c|}{ Dwarfs } \\
\hline HD108519 & $0.43 \pm 0.04$ & $1.61 \pm 0.01$ & $0.06 \pm 0.01$ & $0.51 \pm 0.01$ & $3.40 \pm 0.03$ \\
\hline HD213135 & $0.40 \pm 0.05$ & $1.19 \pm 0.02$ & $0.24 \pm 0.02$ & $0.37 \pm 0.01$ & $2.86 \pm 0.02$ \\
\hline HD113139 & $0.34 \pm 0.03$ & $1.34 \pm 0.02$ & $0.29 \pm 0.02$ & $0.40 \pm 0.02$ & $2.88 \pm 0.05$ \\
\hline HD026015 & $0.44 \pm 0.03$ & $1.21 \pm 0.02$ & $0.34 \pm 0.02$ & $0.53 \pm 0.03$ & $2.80 \pm 0.08$ \\
\hline$\ldots$ & $\ldots$ & $\ldots$ & $\ldots$ & $\ldots$ & $\ldots$ \\
\hline
\end{tabular}




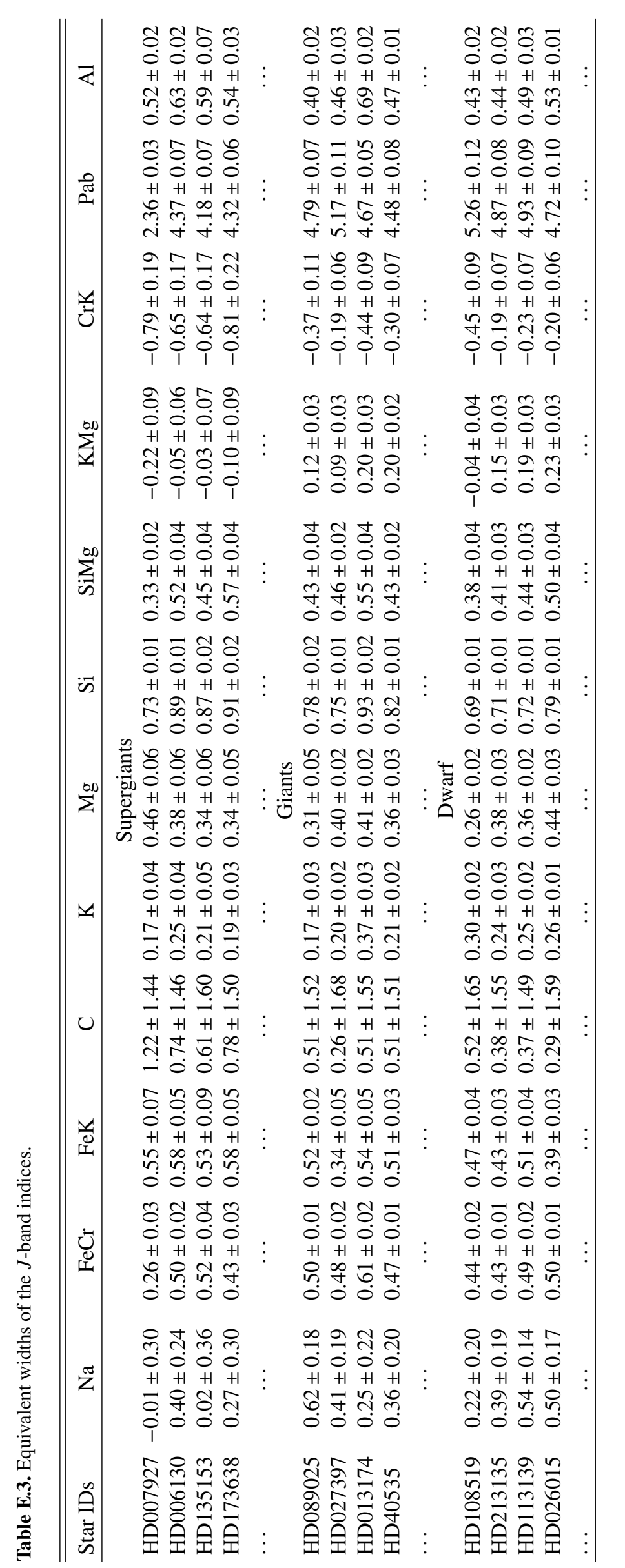

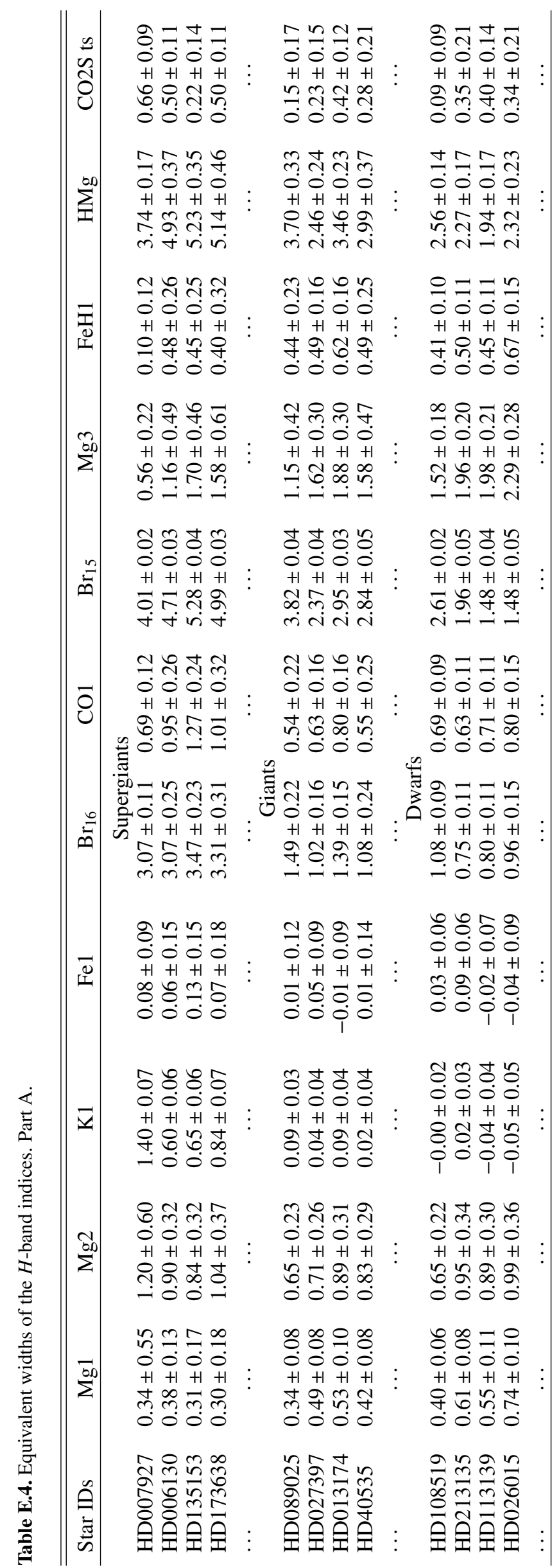


L. Morelli et al.: The Infrared Telescope Facility (IRTF) spectral library. II.

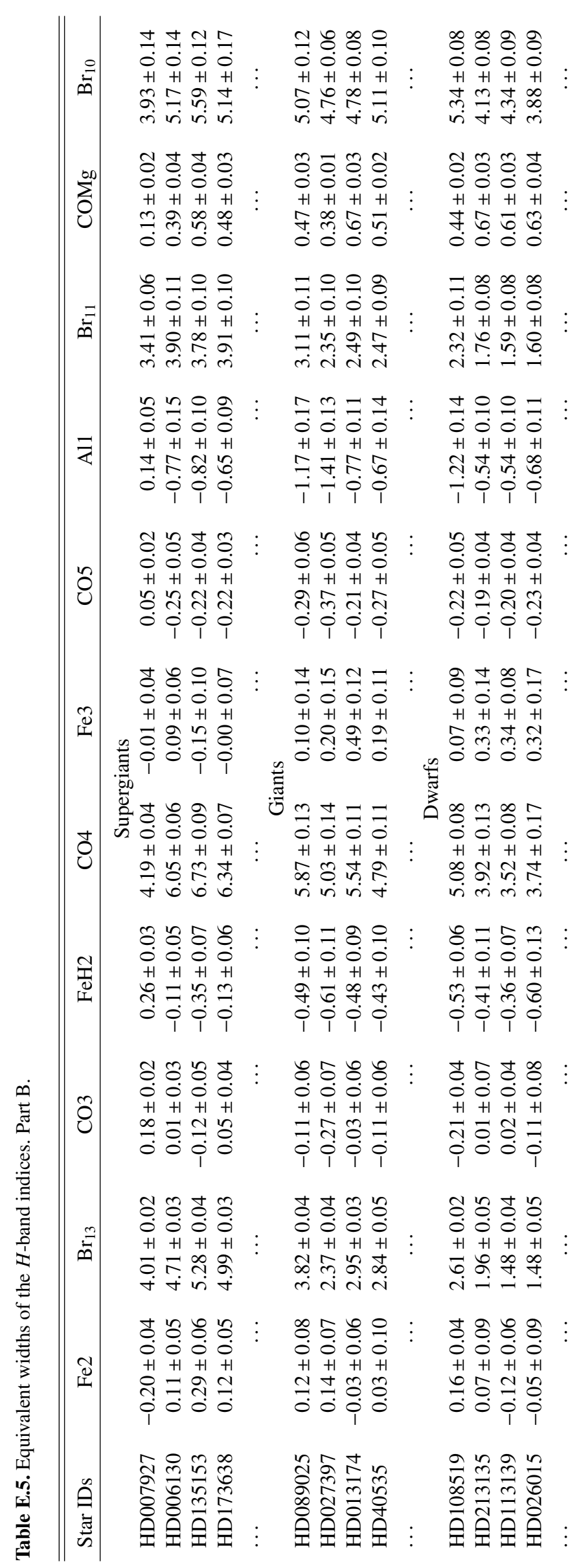

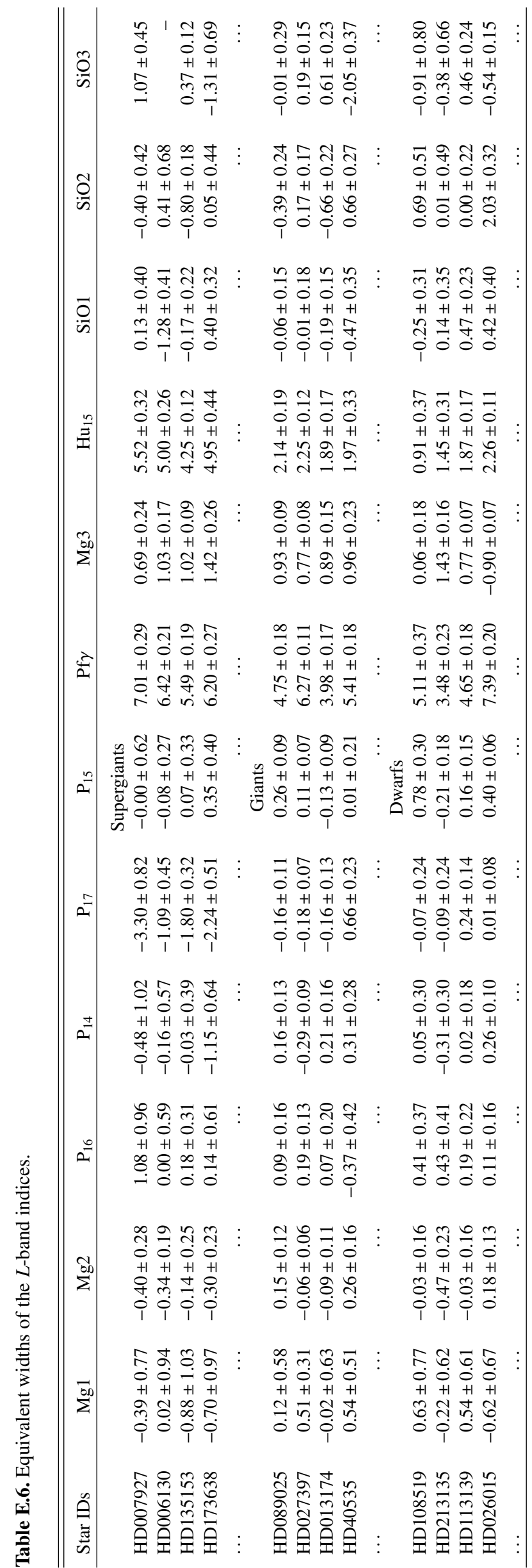

